\title{
WEAKLY-EXCEPTIONAL SINGULARITIES IN HIGHER DIMENSIONS
}

\author{
IVAN CHELTSOV AND CONSTANTIN SHRAMOV
}

\begin{abstract}
We show that infinitely many Gorenstein weakly-exceptional quotient singularities exist in all dimensions, we prove a weak-exceptionality criterion for five-dimensional quotient singularities, and we find a sufficient condition for being weakly-exceptional for six-dimensional quotient singularities. The proof is naturally linked to various classical geometrical constructions related to subvarieties of small degree in projective spaces, in particular Bordiga surfaces and Bordiga threefolds.
\end{abstract}

\section{INTRODUCTION}

Linear representations of finite groups induce their action on polynomial functions. Invariant theory studies polynomial functions that are invariant under the transformations from a given linear group. These functions form a ring, which is called a ring of invariants. From the point of view of Algebraic Geometry, the rings of invariants are algebraic counterparts of the quotients of the vector spaces by these groups, which are usually singular spaces and are called quotient singularities.

Finite subgroups in $\mathrm{SL}_{2}(\mathbb{C})$ have been classified long time ago. The corresponding quotients by these groups are famous $\mathbb{A}-\mathbb{D}$ - $\mathbb{E}$ singularities, which are also known by other names (Kleinian singularities, Du Val singularities, rational surface double points, two-dimensional canonical singularities etc). Taking into account the classical double cover $\mathrm{SU}_{2}(\mathbb{C}) \rightarrow \mathrm{SO}_{3}(\mathbb{R})$ and basic representation theory, we see that the singularities of type $\mathbb{A}$ correspond to plane rotations, the singularities of type $\mathbb{D}$ correspond to the groups of symmetries of regular polygons, and the singularities of type $\mathbb{E}$ corresponds to the groups of symmetries of Platonic solids.

Shokurov suggested a higher dimensional generalization of the singularities of type $\mathbb{E}$ and of both types $\mathbb{D}$ and $\mathbb{E}$. He called them exceptional and weakly-exceptional, respectively. It turned out that exceptional and weakly-exceptional singularities are related to the Calabi problem for orbifolds with positive first Chern class (see [10], [11]). Exceptional quotient singularities of dimensions 3, 4, 5 and 6 have been completely classified by Markushevich, Prokhorov, and the authors (see [36], [11], [12]). Moreover, we proved in [12] that seven-dimensional exceptional quotient singularities are "too exceptional" - they simply do not exist. On the other hand, weakly-exceptional quotient singularities have been less popular despite the fact that their definition is much simpler than the definition of exceptional ones. In this paper, we will try to fill this gap.

What is special about singularities of types $\mathbb{D}$ and $\mathbb{E}$ and how to generalize them to higher dimensions? There are many possible answers to these questions. One of them involves the dual graphs of their minimal resolutions of singularities. Namely, the dual graphs of the minimal resolution of singularities of any two-dimensional quotient singularity of type $\mathbb{D}$ and $\mathbb{E}$ always have a "fork", i.e. a special curve that intersects three other exceptional curves in the graph. The singularities of type $\mathbb{A}$ lack this property. Surprisingly, this property of having a "very special" exceptional divisor on some resolution of singularities can be generalized to higher dimensions for quotient singularities and other "mild" singularities.

Unless explicitly stated otherwise, varieties are assumed to be projective, normal and complex. Throughout the paper we use the standard language of the singularities of pairs (see 33]). By strictly log canonical singularities we mean log canonical singularities that are not Kawamata log terminal (see 33, Definition 3.5]). 
Let $(V \ni O)$ be a germ of a Kawamata log terminal singularity (see [33, Definition 3.5]). Then there exists (see, for example, [11, Theorem 3.6]) a birational morphism $\pi: W \rightarrow V$ whose exceptional locus consists of a single irreducible divisor $E \subset W$ such that $O \in \pi(E)$, the log pair $(W, E)$ has purely log terminal singularities (see [33, Definition 3.5]), and $-E$ is a $\pi$-ample $\mathbb{Q}$ Cartier divisor. The birational morphism $\pi: W \rightarrow V$ is said to be a plt blow up of the singularity $(V \ni O)$.

Example 1.1. Suppose that $(V \ni O)$ is a two-dimensional Du Val singularity. If it is of type $\mathbb{A}$, then let us choose $\pi: W \rightarrow V$ to be any partial resolution of singularities that contracts exactly one curve to the point $O$. In the case when $(V \ni O)$ is a singularity of type $\mathbb{D}$ or $\mathbb{E}$, let us choose $\pi: W \rightarrow V$ to be the partial resolution of singularities contracting exactly one curve that corresponds to the "central" vertex of the dual graph of the minimal resolution of singularities of $(V \ni O)$ to the point $O$. Then $\pi$ is a plt blow up of the singularity ( $V \ni O)$ (cf. Example 1.7).

Example 1.2. Suppose that $(V \ni O)$ is an isolated quasihomogeneous hypersurface singularity in $\mathbb{C}^{n+1}$ with respect to some positive integral weights $\left(a_{0}, \ldots, a_{n}\right)$ such that $\operatorname{gcd}\left(a_{0}, \ldots, a_{n}\right)=1$. Then $(V, O)$ is given by

$$
\phi\left(x_{0}, \ldots, x_{n}\right)=0 \subset \mathbb{C}^{n+1} \cong \operatorname{Spec}\left(\mathbb{C}\left[x_{0}, \ldots, x_{n}\right]\right)
$$

for some quasihomogeneous polynomial $\phi \in \mathbb{C}\left[x_{0}, \ldots, x_{n}\right]$ of degree $d$ with respect to the weights $\operatorname{wt}\left(x_{1}\right)=a_{1}, \ldots, \operatorname{wt}\left(x_{n}\right)=a_{n}$. It is well-known that $(V \ni O)$ is Kawamata log terminal if and only if $\sum_{i=0}^{n} a_{i}>d$. If this is the case, the weighted blow up of $\mathbb{C}^{n+1}$ with weights $\left(a_{0}, \ldots, a_{n}\right)$ induces a plt blow up $\pi$ of $(V \ni O)$. If $n=1$ and $(V \ni O)$ is of type $\mathbb{D}$ or $\mathbb{E}$, then the choice of weights is unique, and the morphism $\pi$ constructed in this example coincides with the morphism $\pi$ constructed in Example 1.1

Example 1.3. Suppose $(V \ni O)$ is a quotient singularity $\mathbb{C}^{n+1} / G$, where $n \geqslant 1$ and $G$ is a finite subgroup in $\mathrm{GL}_{n+1}(\mathbb{C})$. Note that quotient singularities are always Kawamata log terminal (see [32, Remark 0.2.17]). Let $\eta: \mathbb{C}^{n+1} \rightarrow V$ be the quotient map. Then there is a commutative diagram

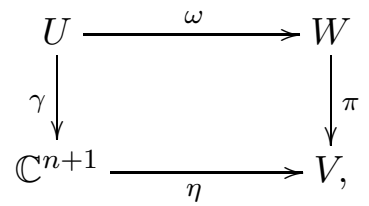

where $\gamma$ is the blow up of $O$, the morphism $\omega$ is the quotient map that is induced by the lifted action of $G$ on the variety $U$, and $\pi$ is a birational morphism. One can easily check that $\pi$ is a plt blow up of the singularity $\mathbb{C}^{n+1} / G$. Note that $\pi$ may not "improve" singularities of $V$. For example, if $n=1, G \subset \mathrm{SL}_{2}(\mathbb{C})$ and $G \cong \mathbb{Z}_{3}$, then this construction does not give a partial resolution of the singularity $(V \ni O)$. However, if $n=1, G \subset \mathrm{SL}_{2}(\mathbb{C})$ and $(V \ni O)$ is a singularity of type $\mathbb{D}$ or $\mathbb{E}$, then the morphism $\pi$ constructed in this example coincides with the morphism $\pi$ constructed in Examples 1.1 and 1.2

Keeping in mind Examples 1.1, 1.2, and 1.3, one gives

Definition 1.4 ([45, Definition 4.1]). We say that $(V \ni O)$ is weakly-exceptional if it has a unique plt blow up.

Note that weakly-exceptional Kawamata log terminal singularities do exist (see Example 1.7). How to decide whether the singularity $(V \ni O)$ is weakly-exceptional or not? Surprisingly, the answer to this question depends only on the log pair $(W, E)$. For instance, a necessary condition for $(V \ni O)$ to be weakly-exceptional says that $\pi(E)=O$ (see [34, Corollary 1.7]). However, it follows from Example 1.1 that this condition is very far from being a criterion. To give a criterion 
for $(V \ni O)$ to be weakly-exceptional, we must equip $E$ with an extra structure. Namely, let $R_{1}, \ldots, R_{s}$ be all irreducible components of the locus $\operatorname{Sing}(W)$ of $\operatorname{dimension} \operatorname{dim}(W)-2$ that are contained in E. Put

$$
\operatorname{Diff}_{E}(0)=\sum_{i=1}^{s} \frac{m_{i}-1}{m_{i}} R_{i},
$$

where $m_{i}$ is the smallest positive integer such that $m_{i} E$ is Cartier at a general point of $R_{i}$. The divisor Diff $E_{E}(0)$ is usually called a different (it was introduced by Shokurov in [53]). It follows from [33, Theorem 7.5] that $E$ is a normal variety that has at most rational singularities, and the log pair $\left(E, \operatorname{Diff}_{E}(0)\right)$ is Kawamata log terminal. Thus, if $\pi(E)=O$, then the log pair $\left(E, \operatorname{Diff}_{E}(0)\right)$ is a log Fano variety, because $-E$ is $\pi$-ample.

Theorem 1.5 (45, Theorem 4.3], [34, Theorem 2.1]). The singularity $(V \ni O)$ is weaklyexceptional if and only if $\pi(E)=O$ and the log pair $\left(E\right.$, Diff $\left.E_{E}(0)+D_{E}\right)$ is $\log$ canonical for every effective $\mathbb{Q}$-divisor $D_{E}$ on the variety $E$ such that $D_{E} \sim_{\mathbb{Q}}-\left(K_{E}+\operatorname{Diff}_{E}(0)\right)$.

Let us translate the assertion of Theorem 1.5 into a slightly different language that uses a global log canonical threshold, that is an algebraic counterpart of the so-called $\alpha$-invariant of Tian introduced in [57].

Remark 1.6. For a log Fano variety $\left(X, B_{X}\right)$ with at most Kawamata log terminal singularities and a finite group $\bar{G} \subset \operatorname{Aut}(X)$ such that $B_{X}$ is $\bar{G}$-invariant, the number

$$
\sup \left\{\begin{array}{l|l}
\lambda \in \mathbb{Q} & \begin{array}{l}
\left(X, B_{X}+\lambda D_{X}\right) \text { has Kawamata log terminal singularities } \\
\text { for every } \bar{G} \text {-invariant } \mathbb{Q} \text {-Cartier effective } \mathbb{Q} \text {-divisor } D_{X} \\
\text { on the variety } X \text { such that } D_{X} \sim_{\mathbb{Q}}-\left(K_{X}+B_{X}\right)
\end{array}
\end{array}\right\} .
$$

is denoted by $\operatorname{lct}\left(X, B_{X}, \bar{G}\right)$ and is called the global $\bar{G}$-invariant log canonical threshold of the $\log$ Fano variety $\left(X, B_{X}\right)$ (see [11, Definition 3.1]). For simplicity, we put $\operatorname{lct}(X, \bar{G})=\operatorname{lct}\left(X, B_{X}, \bar{G}\right)$ if $B_{X}=0$, we put $\operatorname{lct}\left(X, B_{X}\right)=\operatorname{lct}\left(X, B_{X}, \bar{G}\right)$ if $\bar{G}$ is trivial, and we $\operatorname{put} \operatorname{lct}(X)=\operatorname{lct}\left(X, B_{X}, \bar{G}\right)$ if $B_{X}=0$ and $\bar{G}$ is trivial. If $X$ is smooth and $B_{X}=0$, then it follows from [9, Theorem A.3] that $\operatorname{lct}(X, \bar{G})=\alpha_{\bar{G}}(X)$, where $\alpha_{\bar{G}}(X)$ is the $\alpha$-invariant of Tian of the Fano variety $X$. If $X$ has at most quotient singularities and $B_{X}=0$, then it follows from [57, [40] and [16] that $X$ admits a $\bar{G}$-invariant orbifold Kähler-Einstein metric if $\operatorname{lct}(X, G)>\operatorname{dim}(X) /(\operatorname{dim}(X)+1)$.

It follows from Theorem 1.5 that $(V \ni O)$ is weakly-exceptional if and only if $\pi(E)=O$ and $\operatorname{lct}\left(E, \operatorname{Diff}_{E}(0)\right) \geqslant 1$. It should be pointed out that Theorem 1.5 is an applicable criterion. For instance, it can be used to construct weakly-exceptional singularities of any dimension (cf. [11, Example 3.13]).

Example 1.7. In the notation and assumptions of Example 1.1, let $E$ be the exceptional curve of the plt blow up $\pi: W \rightarrow V$. If $(V \ni O)$ is a singularity of type $\mathbb{A}$, then one can easily see that $\operatorname{lct}\left(E, \operatorname{Diff}_{E}(0)\right)<1$, which implies that $(V \ni O)$ is not weakly-exceptional by Theorem 1.5. Suppose that $(V \ni O)$ is a singularity of type $\mathbb{D}$ or $\mathbb{E}$. Then $W$ is singular along $E$, and $E$ contains exactly three singular points of the surface $W$. Let us denote these points by $P_{1}, P_{2}, P_{3}$. Then each $P_{i}$ is a singular point of type $\mathbb{A}_{n_{i}}$ for some non-negative integer $n_{i}$. Without loss of generality, we may assume that $1 \leqslant n_{1} \leqslant n_{2} \leqslant n_{3}$. Then $\operatorname{Diff}_{E}(0)=\sum_{i=1}^{3} \frac{n_{i}}{n_{i}+1} P_{i}$ (see [15. Proposition 16.6]). The log pair $\left(E, \operatorname{Diff}_{E}(0)\right)$ is Kawamata log terminal, since $n_{i} /\left(n_{i}+1\right)<1$ for every $i \in\{1,2,3\}$. Moreover, we have $\sum_{i=1}^{3} \frac{n_{i}}{n_{i}+1}<2$, which implies that $\left(E, \operatorname{Diff}_{E}(0)\right)$ is a 
$\log$ Fano variety. Then

$$
\operatorname{lct}\left(E, \operatorname{Diff}_{E}(0)\right)=\frac{1-\frac{n_{3}}{n_{3}+1}}{2-\sum_{i=1}^{3} \frac{n_{i}}{n_{i}+1}}=\left\{\begin{array}{l}
6 \text { if }(V \ni O) \text { is a singularity of type } \mathbb{E}_{8}, \\
3 \text { if }(V \ni O) \text { is a singularity of type } \mathbb{E}_{7}, \\
2 \text { if }(V \ni O) \text { is a singularity of type } \mathbb{E}_{6}, \\
1 \text { if }(V \ni O) \text { is a singularity of type } \mathbb{D},
\end{array}\right.
$$

which implies that $(V \ni O)$ is weakly-exceptional 1

As it clearly follows from Example 1.7, weakly-exceptional singularities are a natural generalization of Du Val singularities of type $\mathbb{D}_{n}, \mathbb{E}_{6}, \mathbb{E}_{7}$ and $\mathbb{E}_{8}$. On the other hand, weakly-exceptional singularities are not classified even in dimension three. In fact, weakly-exceptional singularities are classified just in two cases. Firstly, three-dimensional weakly-exceptional Kawamata log terminal isolated quasihomogeneous well-formed hypersurface singularities (see [10]), which provides a lot of examples of Kähler-Einstein two-dimensional Fano orbifolds that are hypersurfaces in weighted projective spaces (see Example 1.2 and Remark 1.6). Secondly, three-dimensional and four-dimensional weakly-exceptional quotient singularities are classified in [36, [11] and [51] (cf. Theorems 1.9 and 1.10). The goal of this paper, as it follows from its title, is to study weakly-exceptional quotient singularities in higher dimensions.

Let $G$ be a finite subgroup in $\mathrm{GL}_{n+1}(\mathbb{C})$, where $n \geqslant 1$. Let $\phi: \mathrm{GL}_{n+1}(\mathbb{C}) \rightarrow \mathrm{PGL}_{n+1}(\mathbb{C})$ be the natural projection. Put $\bar{G}=\phi(G)$ and let us identify the group $\mathrm{PGL}_{n+1}(\mathbb{C})$ with $\operatorname{Aut}\left(\mathbb{P}^{n}\right)$. Recall that an element $g \in G$ is called a reflection (or sometimes a quasi-reflection) if there is a hyperplane in $\mathbb{P}^{n}$ that is pointwise fixed by $\phi(g)$. To study the weak-exceptionality of the singularity $\mathbb{C}^{n+1} / G$ one can always assume that the group $G$ does not contain reflections (cf. [12, Remark 1.16]). In this case we have the following

Theorem 1.8 ([11, Theorem 3.16]). Let $G$ be a finite subgroup in $\mathrm{GL}_{n+1}(\mathbb{C})$ that does not contain reflections. Then the singularity $\mathbb{C}^{n+1} / G$ is weakly-exceptional $\Longleftrightarrow \operatorname{lct}\left(\mathbb{P}^{n}, \bar{G}\right) \geqslant 1$.

It should be pointed out that the assumption that $G$ contains no reflections is crucial for Theorem 1.8 and can not be removed (see [11, Example 1.18]). Recall that a semi-invariant of the group $G$ is a polynomial whose zeroes define a $\bar{G}$-invariant hypersurface in $\mathbb{P}^{n}$. If the group $G$ has a semi-invariant of degree $d$, then $\operatorname{lct}\left(\mathbb{P}^{n}, \bar{G}\right) \leqslant d /(n+1)$. Thus, if $G$ does not contain reflections and $G$ has a semi-invariant of degree at most $n$, then $\mathbb{C}^{n+1} / G$ is not weakly-exceptional by Theorem 1.8. Moreover, it follows from Theorem 1.8 and Example 1.7 that if $G$ is a finite subgroup in $\mathrm{GL}_{2}(\mathbb{C})$ that does not contain reflections, then the singularity $\mathbb{C}^{2} / G$ is exceptional if and only if $G$ has no semi-invariants of degree 1. A similar result holds in dimension 3 due to

Theorem 1.9 ([11, Theorem 3.23]). Let $G$ be a finite group in $\mathrm{GL}_{3}(\mathbb{C})$ that does not contain reflections. Then the singularity $\mathbb{C}^{3} / G$ is weakly-exceptional if and only if $G$ does not have semi-invariants of degree at most 2 .

Representation theory provides various obstructions to weak exceptionality. For example, it follows from [46, Proposition 2.1] that the subgroup $G \subset \mathrm{GL}_{n+1}(\mathbb{C})$ is transitive (i. e. the corresponding $(n+1)$-dimensional representation is irreducible) provided that the singularity $\mathbb{C}^{n+1} / G$ is weakly exceptional. Nevertheless, we do not expect that Definition 1.4 can be translated into the representation-theoretic language in higher dimensions even in a very restrictive

\footnotetext{
${ }^{1}$ Let $(V \ni O)$ be a germ of a Kawamata log terminal surface singularity. Then either $(V \ni O)$ is smooth, or the dual graph of its minimal resolution of singularities is of $\mathbb{A}, \mathbb{D}$ or $\mathbb{E}$ type (see [7] and [26] for details). Arguing as in the Du Val case we see that the singularity $(V \ni O)$ is weakly-exceptional if and only if the dual graph is of $\mathbb{D}$ or $\mathbb{E}$ type.

Moreover, it follows from [29, Corollary 1.9] that $(V \ni O)$ is a quotient singularity $\mathbb{C}^{2} / G$ for some finite group $G \subset \mathrm{GL}_{2}(\mathbb{C})$. However, it is not easy to give a nice description of such $G$ that correspond to weakly-exceptional singularities $(V \ni O)$ (cf. [12, Example 1.9]).
} 
case of quotient singularities. Namely, $\bar{G}$-invariant subvarieties of large codimension may also provide obstructions to weak exceptionality as follows from

Theorem 1.10 ([11, Theorem 4.3]). Let $G$ be a finite group in $\mathrm{GL}_{4}(\mathbb{C})$ that does not contain reflections. Then the singularity $\mathbb{C}^{4} / G$ is weakly-exceptional if and only if the following three conditions are satisfied: the group $G$ is transitive, the group $G$ does not have semi-invariants of degree at most 3 , and there is no $\bar{G}$-invariant smooth rational cubic curve in $\mathbb{P}^{3}$.

Futhermore, we can construct a non-weakly exceptional six-dimensional quotient singularity arising from a transitive finite subgroup without reflections in $\mathrm{GL}_{6}(\mathbb{C})$ that has no semi-invariants of degree at most 6 (see [11, Example 3.20] and [11, Lemma 3.21]). This, together with Theorems 1.9 and 1.10, shows that the weak-exceptionality of a quotient singularity $\mathbb{C}^{n+1} / G$ crucially depends on the absence of certain special $\bar{G}$-invariant subvarieties in $\mathbb{P}^{n}$. Note that the transitivity of the subgroup $G$ is equivalent to the absence of $\bar{G}$-invariant proper linear subspaces in $\mathbb{P}^{n}$. In particular, we should not expect that there exists a simple looking criterion for a quotient singularity $\mathbb{C}^{n+1} / G$ to be weakly-exceptional that works in all dimensions. On the other hand, it is quite natural to expect that there exists such a sufficient condition (not a criterion). This is indeed the case.

Definition 1.11. An irreducible normal variety $V$ is said to be of Fano type if there exists an effective $\mathbb{Q}$-divisor $\Delta_{V}$ on the variety $V$ such that $-\left(K_{V}+\Delta_{V}\right)$ is a $\mathbb{Q}$-Cartier ample divisor, and the log pair $\left(V, \Delta_{V}\right)$ has at most Kawamata log terminal singularities (see [33, Definition 3.5]).

In this paper (see Section 2), we prove the following

Theorem 1.12. Let $G$ be a finite group in $\mathrm{GL}_{n+1}(\mathbb{C})$ that does not contain reflections. If $\mathbb{C}^{n+1} / G$ is not weakly-exceptional, then there is a $\bar{G}$-invariant, irreducible, normal, Fano type projectively normal subvariety $V \subset \mathbb{P}^{n}$ such that

$$
\operatorname{deg}(V) \leqslant\left(\begin{array}{c}
n \\
\operatorname{dim}(V)
\end{array}\right)
$$

and for every $i \geqslant 1$ and for every $m \geqslant 0$, we have $h^{i}\left(\mathcal{O}_{\mathbb{P}^{n}}(m) \otimes \mathcal{I}_{V}\right)=h^{i}\left(\mathcal{O}_{V}(m)\right)=0$, and

$$
h^{0}\left(\mathcal{O}_{\mathbb{P}^{n}}((\operatorname{dim}(V)+1)) \otimes \mathcal{I}_{V}\right) \geqslant\left(\begin{array}{c}
n \\
\operatorname{dim}(V)+1
\end{array}\right)
$$

where $\mathcal{I}_{V}$ is the ideal sheaf of the subvariety $V \subset \mathbb{P}^{n}$. Let $\Pi$ be a general linear subspace in $\mathbb{P}^{n}$ of codimension $k \leqslant \operatorname{dim}(V)$. Put $X=V \cap \Pi$. Then $h^{i}\left(\mathcal{O}_{\Pi}(m) \otimes \mathcal{I}_{X}\right)=0$ for every $i \geqslant 1$ and $m \geqslant k$, where $\mathcal{I}_{X}$ is the ideal sheaf of the subvariety $X \subset \Pi$. Moreover, if $k=1$ and $\operatorname{dim}(V) \geqslant 2$, then $X$ is irreducible, projectively normal and $h^{i}\left(\mathcal{O}_{X}(m)\right)=0$ for every $i \geqslant 1$ and $m \geqslant 1$.

Corollary 1.14. Let $G$ be a finite subgroup in $\mathrm{GL}_{n}(\mathbb{C})$ that does not contain reflections. Then the singularity $\mathbb{C}^{n} / G$ is weakly-exceptional if for every irreducible $\bar{G}$-invariant subvariety $V \subset \mathbb{P}^{n}$ there exists no hypersurface in $\mathbb{P}^{n}$ of degree $\operatorname{dim}(V)+1$ that contains $V$.

Apart from their clear geometric nature, Theorem 1.12 and Corollary 1.14 are easy to apply in many cases (but not always, since Theorem 1.12 is not a criterion). In Section 3, we will use them to construct many explicit infinite series of Gorenstein weakly-exceptional quotient singularities. In particular, we will use Corollary 1.14 to prove that infinitely many Gorenstein weakly-exceptional quotient singularities exist in any dimension (see Theorem 3.1 and Corollary 3.2 ) and to prove

Theorem 1.15. Let $p \geqslant 3$ be a prime number, and let $G$ be a subgroup in $\mathrm{SL}_{p}(\mathbb{C})$ that is isomorphic to the Heisenberg group of order $p^{3}$. Then $\mathbb{C}^{p} / G$ is weakly-exceptional. 
We prove Theorem 1.15 (after stating it a bit more explicitely) in Section 3 (see Theorem 3.4). Theorem 1.15 gives many examples of weakly exceptional singularities in dimension $p$ corresponding to the groups containing the group $G$ (cf. Theorem 1.19). Another reason to study weak exceptionality of the quotient by the Heisenberg group is that the order of this group is relatively small compared to that of the groups considered in Theorem 3.1

We have already seen that being weakly-exceptional is not so easy to check for a higher dimensional quotient singularity. This is not surprising: the life is not easy in higher dimensions. Surprisingly, the life is still easy in dimension five as it follows from

Theorem 1.16 (cf. Theorem [1.10). Let $G$ be a finite subgroup in $\mathrm{GL}_{5}(\mathbb{C})$ that does not contain reflections. Then the singularity $\mathbb{C}^{5} / G$ is weakly-exceptional if and only if the group $G$ is transitive and does not have semi-invariants of degree at most 4.

However, in dimension six, nature takes its revenge. In this case we can only prove a sufficient condition.

Theorem 1.17. Let $G$ be a finite subgroup in $\mathrm{GL}_{6}(\mathbb{C})$ that does not contain reflections. Then the singularity $\mathbb{C}^{6} / G$ is weakly-exceptional if the following five conditions are satisfied:

(1) the group $G$ is transitive,

(2) the group $G$ does not have semi-invariants of degree at most 5 ,

(3) there is no irreducible $\bar{G}$-invariant smooth rational cubic scroll $\sqrt{2}$ in $\mathbb{P}^{5}$,

(4) there is no irreducible $\bar{G}$-invariant complete intersection of two quadric hypersurfaces in $\mathbb{P}^{5}$

(5) there is no irreducible $\bar{G}$-invariant, normal, projectively normal, non-degenerate, Fano type threefold $X \subset \mathbb{P}^{5}$ with at most rational singularities of degree 6 and sectional genus 3 such that $h^{0}\left(\mathcal{O}_{\mathbb{P}^{5}}(2) \otimes \mathcal{I}_{X}\right)=0$ and $h^{0}\left(\mathcal{O}_{\mathbb{P}^{5}}(3) \otimes \mathcal{I}_{X}\right)=4$ (cf. Remark 1.18 below).

The main purpose of this paper is to prove Theorems 1.12, 1.16] and 1.17, which is done in Sections 2. 4 and 5, respectively. The proofs of Theorems 1.16 and 1.17 are based on Theorem 1.12 and appear to be naturally linked to many classical geometric constructions, which are very interesting on their own. For example, in the proof of Theorem 1.16 we naturally come across various surfaces of small degree in $\mathbb{P}^{4}$, in particular singular Bordiga surfaces.

It should be pointed out that Theorem 1.16 is indeed a criterion, while Theorems 1.12 and 1.17 provide us only sufficient conditions to be weakly-exceptional. One can easily see that Theorem 1.12 is quite far from being a criterion by comparing it with Theorems 1.9 and 1.10. On the other hand, the first four conditions in Theorem 1.17 are also necessary conditions for the weak-exceptionality (cf. [11, Lemma 3.21]). Unfortunately, we do not know whether the fifth condition in Theorem 1.17 is really necessary or not.

Remark 1.18. Let $X$ be a projectively normal non-degenerate threefold in $\mathbb{P}^{5}$ of degree 6 and sectional genus 3 that has at most Kawamata log terminal singularities. If $X$ is smooth, then it follows from [41, Proposition 1.7] that $X$ is a so-called smooth Bordiga scroll, which is a projectivization of a two-dimensional stable vector bundle $\mathcal{E}$ on $\mathbb{P}^{2}$ such that $\mathrm{c}_{1}(\mathcal{E})=0$ and $\mathrm{c}_{2}(\mathcal{E})=0$. Smooth Bordiga scrolls have been studied in [41, [43] and [38. One can show that smooth Bordiga scrolls are weak Fano threefolds, i.e. their anticanonical divisors are big and nef. Note that smooth Bordiga scrolls are missing in the classifications obtained in [27] and 28]. Smooth hyperplane sections of smooth Bordiga scrolls are known as smooth Bordiga surfaces (see [5], [50, Chapter XIV]), which can be obtained by blowing up $\mathbb{P}^{2}$ at 10 sufficiently general points. It follows from [47] that some smooth Bordiga surfaces in $\mathbb{P}^{4}$ are set-theoretic intersections of a cubic and a quartic hypersurfaces (see also [25, Proposition 19]). If one can

\footnotetext{
${ }^{2}$ This is just $\mathbb{P}^{1} \times \mathbb{P}^{2}$ embedded by Segre.
} 
show that $X$ is also a set-theoretic intersections of a cubic and a quartic hypersurfaces in $\mathbb{P}^{5}$ (without imposing any additional assumption on $X$ except may be those that are used in the fifth condition in Theorem 1.17), then the fifth condition in Theorem 1.17 is also a necessary condition for the singularity $\mathbb{C}^{6} / G$ in Theorem 1.17 to be weakly-exceptional. Unfortunately, we do not even know whether there exists an example of a smooth three-dimensional Bordiga scroll that is a set-theoretic intersections of a cubic and a quartic hypersurfaces. On the other hand, there is a small chance that the fifth condition in Theorem 1.17 follows from the first four, which would imply that it can be dropped from Theorem 1.17.

By Theorem 1.8, to apply Theorems 1.16 and 1.17, we may assume that $G \subset \mathrm{SL}_{n+1}(\mathbb{C})$, since there exists a finite subgroup $G^{\prime} \subset \mathrm{SL}_{n+1}(\mathbb{C})$ such that $\phi\left(G^{\prime}\right)=\bar{G}$. Every transitive finite subgroup of $\mathrm{SL}_{2}(\mathbb{C})$ gives rise to a weakly-exceptional singularity by Theorem 1.8. In [51, Sakovich used Theorems 1.9 and 1.10 to obtain an explicit classification of the transitive finite subgroups in $\mathrm{SL}_{3}(\mathbb{C})$ and $\mathrm{SL}_{4}(\mathbb{C})$ corresponding to three-dimensional and four-dimensional weakly-exceptional quotient singularities, respectively. Probably, a similar classification is possible in dimensions 5 and 6 using Theorems 1.16 and 1.17, respectively. But this task requires huge amount of computations that goes beyond the scope and the purpose of this paper. So instead, let us apply Theorems 1.16 and 1.17 to classify all primitive (see [11, Definition 1.21]) finite subgroups in $\mathrm{SL}_{5}(\mathbb{C})$ and $\mathrm{SL}_{6}(\mathbb{C})$ corresponding to five-dimensional and six-dimensional weakly-exceptional quotient singularities, respectively.

Theorem 1.19 (cf. [11, Theorems 1.22 and 5.6]). Let $G \subset \mathrm{SL}_{5}(\mathbb{C})$ be a finite primitive group. Then the singularity $\mathbb{C}^{5} / G$ is weakly-exceptional if and only if $G$ contains the Heisenberg group of order 125 .

Proof. The "if" part follows immediately by Theorem 1.15. Let us prove the "only if". Suppose that $G$ does not contain the Heisenberg group of order 125. By Theorem 1.8, the weakexceptionality of the singularity $\mathbb{C}^{5} / G$ depends only on the image of the group $G$ in $\operatorname{PSL}_{5}(\mathbb{C})$, so that we may assume that $Z(G) \subset[G, G]$ (see [21]). Then the group $G$ is one of the following groups: $\mathrm{A}_{5}, \mathrm{~A}_{6}, \mathrm{~S}_{5}, \mathrm{~S}_{6}, \mathrm{PSL}_{2}\left(\mathbb{F}_{11}\right)$ or $\mathrm{PSp}_{4}\left(\mathbb{F}_{3}\right)$ (see [21, 88.5$\left.]\right)$. In all of these cases there exists a semi-invariant of $G$ of degree at most 4 by [11, Lemma 5.3], so that the singularity $\mathbb{C}^{5} / G$ is not weakly-exceptional by Theorem 1.8 .

Theorem 1.20 (cf. [12, Theorem 1.14]). Let $G$ be a finite primitive subgroup in $\mathrm{SL}_{6}(\mathbb{C})$. Then the singularity $\mathbb{C}^{6} / G$ is weakly-exceptional if and only if there exists a lift of the subgroup $\bar{G} \subset \mathrm{PGL}_{6}(\mathbb{C})$ to $\mathrm{SL}_{6}(\mathbb{C})$ that is contained in the following list 3 :

(V) $6 . A_{6}$,

(VIII) $6 . \mathrm{A}_{7}$,

(XIV) (i) $\mathrm{SU}_{3}\left(\mathbb{F}_{3}\right)$

(ii) an extension of the subgroup described in XIV(i) by an automorphism of order 2,

$(\mathrm{XV})$ (i) $6 . \mathrm{PSU}_{4}\left(\mathbb{F}_{3}\right)$,

(ii) an extension of the subgroup described in XV(i) by an automorphism of order 2 ,

(XVI) 2.HaJ, where HaJ is the Hall-Janko sporadic simple group,

(XVII) (i) $6 . \mathrm{PSL}_{3}\left(\mathbb{F}_{4}\right)$

(ii) an extension of the subgroup described in XVII(i) by an automorphism of order 2 .

Proof. The classification of the primitive subgroups of $\mathrm{SL}_{6}(\mathbb{C})$ is given in [35, §3]. Browsing through it, we find that primitive subgroups of $\mathrm{SL}_{6}(\mathbb{C})$ that do not have semi-invariants of degree at most 5 are exactly those listed in the assertion of the theorem (see the proof of [12, Theorem 3.3]) and those that satisfy the hypotheses of [11, Lemma 3.24]. If $G$ satisfies the hypotheses of [11, Lemma 3.24], then the singularity $\mathbb{C}^{6} / G$ is not weakly-exceptional by Theorem 1.8 and [11, Lemma 3.24]. If $G$ is of type XIV(i), XIV(ii), XV(i), XV(ii), XVI, XVII(i)

\footnotetext{
${ }^{3}$ We label the cases according to the notation of 21] and 35]. 
and XVII(ii), then $G$ does not have an irreducible representation $W$ such that $2 \leqslant \operatorname{dim}(W) \leqslant 4$ (see [14]), which easily implies that these cases give rise to weakly-exceptional singularities by Theorem 1.17. If $G$ is of type $\mathrm{V}$, then $G \cong 6 . \mathrm{A}_{6}$ has no irreducible two-dimensional representations (see [14]), and no irreducible representation of the group $G$ of dimension 3 or 4 is contained in $\operatorname{Sym}^{3}\left(V^{\vee}\right)$, where $V \cong \mathbb{C}^{6}$ is the $G$-representation in question. The latter can be checked by a direct computation (we used the Magma software [6] to carry it out). Therefore, the singularity $\mathbb{C}^{6} / G$ is again weakly-exceptional by Theorem 1.17 .

It seems possible to apply Theorems 1.15, 1.16, 1.17, 1.19, and1.20 to construct non-conjugate isomorphic finite subgroups in Cremona groups of high ranks (cf. [8, Example 6.5]). For example, if $\operatorname{lct}\left(\mathbb{P}^{n}, \bar{G}\right) \geqslant 1$ and $\mathbb{P}^{n}$ is $\bar{G}$-birationally super-rigid (see [8, Definition 6.1]), then it follows from [8, Theorem 6.4] that there exists no $\bar{G} \times \bar{G}$-equivariant birational map $\mathbb{P}^{n} \times \mathbb{P}^{n} \rightarrow \mathbb{P}^{2 n}$ with respect to the product action of the group $\bar{G} \times \bar{G}$ on $\mathbb{P}^{n} \times \mathbb{P}^{n}$. By Theorem 1.5, we can use Theorems 1.15, 1.16, 1.17, 1.19, and 1.20 to obtain many finite subgroups $\bar{G} \subset \operatorname{Aut}\left(\mathbb{P}^{n}\right)$ with $\operatorname{lct}\left(\mathbb{P}^{n}, \bar{G}\right) \geqslant 1$. However, if $n \geqslant 3$, then it is usually very hard to prove that $\mathbb{P}^{n}$ is $\bar{G}$-birationally super-rigid (cf. [13]). In fact, we do not know any such example if $n \geqslant 4$.

Let us describe the structure of this paper. In Section 2, we prove Theorem 1.12, In Section 3, we construct (see Theorem 3.1) infinite series of Gorenstein weakly-exceptional quotient singularities in any dimension (in particular, proving that weakly-exceptional quotient singularities exist in all dimensions), and prove Theorem 1.15. In Section 4, we prove Theorem 1.16. In Section 5, we prove Theorem 1.17, In Appendix A, we prove an auxiliary statement concerning smooth irreducible curves in $\mathbb{P}^{3}$ of genus 5 and degree 7: we prove that any such curve is a settheoretic intersection of cubics (this result might be known to experts, but we did not manage to find a reference in the literature).

Throughout the proofs of Theorems 1.16 and 1.17 we often work with singular del Pezzo surfaces. A good preliminary reading for the corresponding techniques may be [17. On the other hand, the facts from the theory of representations of finite groups we will need (say, in the proof of Theorem 3.4) do not go beyond the elementary material that can be found in any textbook on the subject (for example, 52]).

The problem of finding a nice geometric criterion for a five-dimensional quotient singularity to be weakly-exceptional originated during the first author participation in the 18th Gökova Conference in Turkey. The first author would like to thank Selman Akbulut for inviting him to this beautiful place. The authors would like to thank Marco Andreatta, Eduardo Ballico, Pietro De Poi, Igor Dolgachev, Stephane Lamy, Jihun Park, Emilia Mezzetti, Yuri Prokhorov and Franchesco Russo for many fruitful discussions.

We proved both Theorems 1.16 and 1.17 while participating in the Research in Groups program in the Center of International Research in Mathematics (Trento, Italy). We finished this paper at the Institute for the Physics and Mathematics of the Universe (Tokyo, Japan). We are really grateful to CIRM and IPMU for the beautiful working conditions. Special thanks goes to Sergey Galkin for his warm and encouraging support during our stay at IPMU. The work was also supported by the grants NSF DMS-1001427, N.Sh.-4713.2010.1, RFFI 11-01-00336-a, RFFI 11-01-92613-KO-a, RFFI 08-01-00395-a, RFFI 11-01-00185-a, and by AG Laboratory GU-HSE, RF government grant 11 11.G34.31.0023.

\section{WEAK-EXCEPTIONALITY CRITERION}

The purpose of this section is to prove Theorem 1.12. Let $G$ be a finite subgroup in $\mathrm{GL}_{n+1}(\mathbb{C})$, and let $\phi: \mathrm{GL}_{n+1}(\mathbb{C}) \rightarrow \mathrm{PGL}_{n+1}(\mathbb{C})$ be the natural projection. Put $\bar{G}=\phi(G)$. Let us identify $\operatorname{PGL}_{n+1}(\mathbb{C})$ with Aut $\left(\mathbb{P}^{n}\right)$. Let us denote by $H$ a general hyperplane in $\mathbb{P}^{n}$. Suppose that $G$ 
contains no reflections and $\mathbb{C}^{n+1} / G$ is not weakly-exceptional. Then $\operatorname{lct}\left(\mathbb{P}^{n}, \bar{G}\right)<1$ by Theorem 1.8. Thus, there is an effective $\bar{G}$-invariant $\mathbb{Q}$-divisor $D$ on $\mathbb{P}^{n}$ such that $D \sim_{\mathbb{Q}}(n+1) H$ and a positive rational number $\lambda<1$ such that $\left(\mathbb{P}^{n}, \lambda D\right)$ is strictly $\log$ canonical.

Let $V$ be a minimal center of log canonical singularities of the log pair $\left(\mathbb{P}^{n}, \lambda D\right)$ (see 30 , Definition 1.3], [31]). Then it follows from the Kawamata subadjunction theorem (see [31, Theorem 1]) that $V$ is normal and has at most rational singularities, and for any rational number $\epsilon<(1-\lambda)(n+1)$ there is an effective $\mathbb{Q}$-divisor $\Delta_{\epsilon}$ on $V$ such that $-\left.\left(K_{V}+\Delta_{\epsilon}\right) \sim_{\mathbb{Q}} \epsilon H\right|_{V}$, and the log pair $\left(V, \Delta_{\epsilon}\right)$ has Kawamata $\log$ terminal singularities. We see that $V$ is a Fano type subvariety. In particular, we see that $h^{i}\left(\mathcal{O}_{V}\left(\left.m H\right|_{V}\right)\right)=0$ for every $i \geqslant 1$ and $m \geqslant 0$ by the Nadel-Shokurov vanishing theorem (see [33, Theorem 2.16] or [2, Appendix]).

Let $Z$ be the $\bar{G}$-orbit of the subvariety $V$, and let $\mu$ be a rational number such that $\mu>1$ and $\mu \lambda<1$. Then it follows from [11, Lemma 2.8] that there exists an effective $\bar{G}$-invariant $\mathbb{Q}$-divisor $D^{\prime}$ on $\mathbb{P}^{n}$ such that $D^{\prime} \sim_{\mathbb{Q}} \mu \lambda D$, the log pair $\left(\mathbb{P}^{n}, D^{\prime}\right)$ is strictly log canonical, and every $\log$ canonical center of the $\log$ pair $\left(\mathbb{P}^{n}, D^{\prime}\right)$ is a component of $Z$. Since $\mu \lambda<1$, it follows from the Nadel-Shokurov vanishing theorem that $h^{i}\left(\mathcal{I}_{Z} \otimes \mathcal{O}_{\mathbb{P}^{n}}(m)\right)=0$ for every $i \geqslant 1$ and $m \geqslant 0$, where $\mathcal{I}_{Z}$ is an ideal sheaf of $Z$. Thus, the sequence of cohomology groups

$$
0 \longrightarrow H^{0}\left(\mathcal{O}_{\mathbb{P}^{n}}(m) \otimes \mathcal{I}_{Z}\right) \longrightarrow H^{0}\left(\mathcal{O}_{\mathbb{P}^{n}}(m)\right) \longrightarrow H^{0}\left(\mathcal{O}_{V}\left(\left.m H\right|_{Z}\right)\right) \longrightarrow 0
$$

is exact for every $m \geqslant 0$, which implies $Z$ is connected, and $V$ is projectively normal if $V=Z$. Since $Z$ is, one has $V=Z$ by [30, Proposition 1.5].

Put $d=\operatorname{dim}(V)$. Let $\Pi$ be a general linear subspace in the projective space $\mathbb{P}^{n}$ of codimension $k \leqslant d$. Put $D_{\Pi}^{\prime}=\left.D^{\prime}\right|_{\Pi}$. Then every center of log canonical singularities ofthe log pair ( $\left.\Pi, D_{\Pi}^{\prime}\right)$ is a components of the intersection $V \cap \Pi$, since $\Pi$ is sufficiently general. Put $H_{\Pi}=\left.H\right|_{\Pi}$ and $X=\Pi \cap V$. Then

$$
K_{\Pi}+D_{\Pi}^{\prime} \sim_{\mathbb{Q}}(\mu \lambda(n+1)-n+k-1) H_{\Pi},
$$

where $\mu \lambda<1$. Thus, it follows from the Nadel-Shokurov vanishing theorem that

$$
h^{i}\left(\mathcal{O}_{\Pi}\left(m H_{\Pi}\right) \otimes \mathcal{I}_{X}\right)=0
$$

for every $i \geqslant 1$ and $m \geqslant k$, where $\mathcal{I}_{X}$ is the ideal sheaf of the subvariety $X \subset \Pi$. If we put $k=d$, then it follows from (2.1) that

$$
\operatorname{deg}(V)=|V \cap \Pi|=h^{0}\left(\mathcal{O}_{X}\right)=h^{0}\left(\mathcal{O}_{\Pi}\left(d H_{\Pi}\right)\right)-h^{0}\left(\mathcal{O}_{\Pi}\left(H_{\Pi}\right) \otimes \mathcal{I}_{X}\right) \leqslant\left(\begin{array}{l}
n \\
d
\end{array}\right) .
$$

Suppose that $k=1$ and $d \geqslant 2$. Then $X$ is irreducible and the sequence

$$
0 \longrightarrow H^{0}\left(\mathcal{O}_{\Pi}\left(m H_{\Pi}\right) \otimes \mathcal{I}_{X}\right) \longrightarrow H^{0}\left(\mathcal{O}_{\Pi}\left(m H_{\Pi}\right)\right) \longrightarrow H^{0}\left(\mathcal{O}_{X}\left(\left.m H\right|_{X}\right)\right) \longrightarrow 0
$$

is exact for every $m \geqslant k=1$ by (2.1), which implies that $X \subset \Pi$ is projectively normal.

It follows from (2.1) that $h^{i}\left(\mathcal{O}_{X}\left(\left.m H\right|_{X}\right)\right)=h^{i}\left(\mathcal{O}_{\Pi}\left(m H_{\Pi}\right)\right)=0$ for every $i \geqslant 1$ and $m \geqslant 1$, since $h^{i}\left(\mathcal{O}_{\Pi}\left(m H_{\Pi}\right)\right)=0$ for every $i \geqslant 1$ and $m \geqslant 0$.

Let $\Lambda$ be a general linear subspace in $\mathbb{P}^{n}$ of codimension $d+1$, let $N$ be a very big integer, and let $H_{1}, H_{2}, \ldots, H_{N}$ be sufficiently general hyperplanes in $\mathbb{P}^{n}$ that contain $\Lambda$. Then

$$
\left(\mathbb{P}^{n}, D^{\prime}+\frac{d+1}{N} \sum_{i=1}^{N} H_{i}\right)
$$

is strictly log canonical. Moreover, it follows from the construction of the log pair (2.2) that the only centers of $\log$ canonical singularities of the $\log$ pair (2.2) are $V$ and $\Lambda$. Note that $V \cap \Lambda=\varnothing$ by construction. Put $m=d+1$. Then it follows from the Nadel-Shokurov vanishing theorem that

$$
h^{0}\left(\mathcal{O}_{\mathbb{P}^{n}}(m) \otimes \mathcal{I}_{V \cup \Lambda}\right)=\left(\begin{array}{c}
n+m \\
m
\end{array}\right)-h^{0}\left(\mathcal{O}_{V}\left(\left.m H\right|_{V}\right)\right)-\left(\begin{array}{c}
n \\
m
\end{array}\right),
$$


since $V \cap \Lambda=\varnothing$. Thus, it follows from the projective normality of the subvariety $V \subset \mathbb{P}^{n}$ that

$$
h^{0}\left(\mathcal{O}_{\mathbb{P}^{n}}(m) \otimes \mathcal{I}_{V}\right)=\left(\begin{array}{c}
n+m \\
m
\end{array}\right)-h^{0}\left(\mathcal{O}_{V}\left(\left.m H\right|_{V}\right)\right)=\left(\begin{array}{c}
n \\
m
\end{array}\right)+h^{0}\left(\mathcal{O}_{\mathbb{P}^{n}}(m) \otimes \mathcal{I}_{V \cup \Lambda}\right),
$$

which implies the inequality (1.13) (cf. the proof of [40, Theorem 6.1]) and completes the proof of Theorem 1.12 .

\section{INFINITE SERIES}

Let $G$ be a finite subgroup in $\mathrm{GL}_{n+1}(\mathbb{C})$, and let $\phi: \mathrm{GL}_{n+1}(\mathbb{C}) \rightarrow \mathrm{PGL}_{n+1}(\mathbb{C})$ be the natural projection. Put $\bar{G}=\phi(G)$ and $V=\mathbb{C}^{n+1}$, and let us identify $\mathrm{PGL}_{n+1}(\mathbb{C})$ with $\operatorname{Aut}\left(\mathbb{P}^{n}\right)$. Let us fix coordinates $\left(x_{0}, \ldots, x_{n}\right)$ on $V$, and let us use them also as projective coordinates on $\mathbb{P}^{n}$.

Theorem 3.1. Suppose that the group $G$ does not contain reflections, the representation $V$ of the group $G$ is irreducible, and $G$ contains a subgroup $\Gamma \cong \mathbb{Z}_{k}^{n}$ for some integer $k \geqslant n+1$ such that $\Gamma$ is generated by the transformations $\gamma_{1}, \gamma_{2}, \ldots, \gamma_{n}$ defined by

$$
\gamma_{i}:\left(x_{0}, x_{1}, \ldots, x_{i-1}, x_{i}, x_{i+1}, \ldots, x_{n}\right) \mapsto\left(\zeta^{-1} x_{0}, x_{1}, \ldots, x_{i-1}, \zeta x_{i}, x_{i+1}, \ldots, x_{n}\right),
$$

where $\zeta$ is a primitive root of unity of degree $k$. Then the singularity $\mathbb{C}^{n+1} / G$ is weakly exceptional.

Proof. Suppose that the singularity $\mathbb{C}^{n+1} / G$ is not weakly exceptional. Then it follows from Corollary 1.14 that there exists a $\bar{G}$-invariant irreducible subvariety $X \subset \mathbb{P}^{n}$ such that there exists a hypersurface in $\mathbb{P}^{n}$ of degree $\operatorname{dim}(X)+1$ that contains $X$. Let us derive a contradiction. To do this, we may swap $G$ with any other group $G^{\prime} \subset \mathrm{GL}_{n+1}(\mathbb{C})$ such that $\phi\left(G^{\prime}\right)=\bar{G}$. Thus, adding a scalar matrix $\operatorname{diag}(\zeta, \ldots, \zeta)$ to the group $G$, we may assume that $G$ contains a subgroup $\Gamma^{\prime} \cong \mathbb{Z}_{k}^{n+1}$ that is generated by the transformations $\gamma_{0}^{\prime}, \gamma_{1}^{\prime}, \gamma_{2}^{\prime}, \ldots, \gamma_{n}^{\prime}$ such that

$$
\gamma_{i}^{\prime}:\left(x_{0}, \ldots, x_{i-1}, x_{i}, x_{i+1}, \ldots, x_{n}\right) \mapsto\left(x_{0}, \ldots, x_{i-1}, \zeta x_{i}, x_{i+1}, \ldots, x_{n}\right),
$$

where as before $\zeta$ is a primitive root of unity of degree $k$. This assumption is not crucial for the proof, but it makes some steps clearer.

Put $d=\operatorname{dim}(X)+1$. Then $d \leqslant n$. Every $\Gamma^{\prime}$-invariant vector subspace in $\mathbb{C}\left[x_{0}, \ldots, x_{n}\right]$ consisting of forms of degree $d$ splits into a sum of pairwise non-isomorphic one-dimensional representations of the group $\Gamma^{\prime}$ that are generated by monomials. Thus, any non-trivial $\Gamma^{\prime}$-invariant vector subspace in $\mathbb{C}\left[x_{0}, \ldots, x_{n}\right]$ consisting of forms of degree $d$ must contain a monomial. In particular, every semi-invariant form in $\mathbb{C}\left[x_{0}, \ldots, x_{n}\right]$ of degree $d$ with respect to $\Gamma^{\prime}$ must be a monomial. Therefore, the group $G$ does not have semi-invariant form in $\mathbb{C}\left[x_{0}, \ldots, x_{n}\right]$ of degree $d$, because $G$ is transitive. Indeed, if there exists a semi-invariant form in $\mathbb{C}\left[x_{0}, \ldots, x_{n}\right]$ of degree $d$ with respect to $G$, then this form must be a monomial $x_{i_{1}} x_{i_{2}} \ldots x_{i_{d}}$ for some (not necessarily distinct) $i_{1}, i_{2}, \ldots, i_{d}$ in $\{0, \ldots, n\}$, which implies that the vector subspace in $V$ given by $x_{i_{1}}=x_{i_{2}}=\ldots=x_{i_{d}}=0$ must be $G$-invariant and proper, since $d \leqslant n$.

Let $W(X)$ be the vector subspace in $\mathbb{C}\left[x_{0}, \ldots, x_{n}\right]$ consisting of forms of degree $d$ that vanish on $X$. Then $W(X)$ is non-zero and $G$-invariant, because $X$ is $\bar{G}$-invariant. Thus, the vector subspace $W(X)$ must contain a monomial $x_{j_{1}} x_{j_{2}} \ldots x_{j_{d}}$ for some $j_{1}, j_{2}, \ldots, j_{d}$ in $\{0, \ldots, n\}$. Therefore, the subvariety $X$ is contained in the union of hyperplanes in $\mathbb{P}^{n}$ that are given by $x_{j_{1}}=0, x_{j_{2}}=0, \ldots, x_{j_{d}}=0$. Since $X$ is irreducible, the subvariety $X$ is contained in one of these hyperplanes. Without loss of generality, we may assume that $X$ is contained in the hyperplane that is given by $x_{j_{1}}=0$. Therefore, the linear span of the subvariety $X$ is also contained in the hyperplane that is given by $x_{j_{1}}=0$, which is impossible, since $V$ is an irreducible representation of the group $G$.

Corollary 3.2. For any $N>1$ there are infinitely many Gorenstein weakly-exceptional quotient singularities of dimension $N$. 
In the remaining part of this section we will give a proof of Theorem 1.15. In order to do this, we need the following

Lemma 3.3. Let $p \geqslant 2$ be a prime number, let $P(x)$ be a polynomial in $\mathbb{Q}[x]$ of degree $d$. Put

$$
P(x)=\sum_{i=0}^{d} \frac{b_{i}}{c_{i}} x^{i},
$$

where $b_{i}$ and $c_{i}$ integers such that $c_{i} \neq 0$ and $\operatorname{gcd}\left(b_{i}, c_{i}\right)=1$. Take $\gamma \in \mathbb{Z}$. For $i \in\{0, \ldots, d\}$, let $P(\gamma+i)=\frac{r_{i}}{q_{i}}$, where $r_{i}$ and $q_{i}$ are integers such that $q_{i} \neq 0$ and $\operatorname{gcd}\left(r_{i}, q_{i}\right)=1$. Suppose that $r_{0} \equiv r_{1} \equiv \ldots \equiv r_{d} \equiv 0 \bmod p$ and $d<p$. Then $b_{0} \equiv b_{1} \equiv \ldots \equiv b_{d} \equiv 0 \bmod p$.

Proof. For every $i$, put $c_{i}=p^{t_{i}} m_{i}$, where $t_{i}$ is a non-negative integer, and $m_{i}$ is an integer that is not divisible by $p$. Put $t=\max \left(t_{0}, \ldots, t_{d}\right)$, and put $N=\operatorname{lcm}\left(c_{0}, \ldots, c_{d}\right)$. Then one has $N=p^{t} \operatorname{lcm}\left(m_{0}, \ldots, m_{d}\right)$ and $N f(x) \in \mathbb{Z}[x]$. Take any $k \in\{0, \ldots, d\}$ such that $t=t_{k}$. Then

$$
N f(\gamma) \equiv \frac{N r_{0}}{q_{0}} \equiv N f(\gamma+1) \equiv \frac{N r_{1}}{q_{1}} \equiv \ldots \equiv N f(\gamma+d) \equiv \frac{N r_{d}}{q_{d}} \equiv 0 \bmod p,
$$

which immediately implies that all integers $N b_{0} / c_{0}, N b_{1} / c_{1}, \ldots, N b_{d} / c_{d}$ must be divisible by $p$, because only the zero polynomial in $\mathbb{F}_{p}[x]$ of degree $d<p$ has $d+1$ different roots. Then

$$
\frac{N b_{k}}{c_{k}}=\frac{p^{t_{k}} \operatorname{lcm}\left(m_{0}, \ldots, m_{d}\right) b_{k}}{p^{t^{k}} m_{k}}=\frac{\operatorname{lcm}\left(m_{0}, \ldots, m_{d}\right) b_{k}}{m_{k}}
$$

is divisible by $p$, which implies that $b_{k}$ is divisible by $p$. Since $\operatorname{gcd}\left(b_{k}, p^{t_{k}} m_{k}\right)=\operatorname{gcd}\left(b_{k}, c_{k}\right)=1$, we see that $t=t_{k}=0$. Hence, we have $t_{0}=\ldots=t_{d}=0$. Thus, we see that $N$ is not divisible by $p$, which implies that $b_{0} \equiv b_{1} \equiv \ldots \equiv b_{d} \equiv 0 \bmod p$, since we know that all integers $N b_{0} / c_{0}, N b_{1} / c_{1}, \ldots, N b_{d} / c_{d}$ are divisible by $p$.

Now we are ready to prove

Theorem 3.4. Let $p \geqslant 3$ be a prime number. Suppose that $n+1=p$ and $G$ is generated by the elements $\left(x_{0}: x_{1}: \ldots: x_{p-1}\right) \rightarrow\left(x_{1}: \ldots: x_{p-1}: x_{0}\right)$ and

$$
\left(x_{0}: x_{1}: \ldots: x_{p-1}\right) \mapsto\left(x_{0}: \zeta x_{1}: \ldots: \zeta^{p-1} x_{p-1}\right),
$$

where $\zeta$ is a primitive root of unity of degree $p$. Then the singularity $\mathbb{C}^{n+1} / G$ is weakly exceptional.

Proof. Suppose that the singularity $\mathbb{C}^{n+1} / G$ is not weakly exceptional. Then it follows from Theorem 1.12 that there exists a $\bar{G}$-invariant irreducible normal Fano type subvariety $V \subset \mathbb{P}^{n}$ such that

$$
\operatorname{deg}(V) \leqslant\left(\begin{array}{c}
n \\
\operatorname{dim}(V)
\end{array}\right)
$$

and $h^{i}\left(V, \mathcal{O}_{V}(m)\right)=0$ for any $i \geqslant 1$ and any $m \geqslant 0$, where $\mathcal{O}_{V}(m)=\mathcal{O}_{V} \otimes \mathcal{O}_{\mathbb{P}^{n}}(m)$. Then

$$
h^{0}\left(V, \mathcal{O}_{V}(m)\right)=h^{0}\left(\mathbb{P}^{n}, \mathcal{O}_{\mathbb{P}^{n}}(m)\right)-h^{0}\left(\mathbb{P}^{n}, \mathcal{I}_{V}(m)\right),
$$

where $\mathcal{I}_{V}$ is the ideal sheaf of $V$.

Let $Z(G)$ be the center of the group $G$. Then $Z(G) \cong \mathbb{Z}_{p}$. It is well-known that any irreducible representation of $G$ with a non-trivial action of the center $Z(G)$ is $p$-dimensional. In particular, the group $G$ has no semi-invariants of degree less than $p$, which implies that $\operatorname{dim}(V) \leqslant p-2$.

Put $W_{m}=H^{0}\left(\mathbb{P}^{n}, \mathcal{I}_{V}(m)\right)$. Then $W_{m}$ is a linear representation of the group $G$, and $Z(G)$ acts non-trivially on $W_{m}$ for every $m \in\{1, \ldots, p-1\}$. Therefore $\operatorname{dim}\left(W_{m}\right)$ is divisible by $p$ for every $m \in\{1, \ldots, p-1\}$. Applying (3.5) for every $m \in\{1, \ldots, p-1\}$ and keeping in mind that $h^{0}\left(\mathbb{P}^{n}, \mathcal{O}_{\mathbb{P}^{n}}(m)\right)$ is divisible by $p$ for every $m \in\{1, \ldots, p-1\}$, we see that $h^{0}\left(V, \mathcal{O}_{V}(m)\right)$ is 
divisible by $p$ for every $m \in\{1, \ldots, p-1\}$. Put $d=\operatorname{dim}(V)$. Since $h^{0}\left(V, \mathcal{O}_{V}(m)\right)=\chi\left(V, \mathcal{O}_{V}(m)\right)$ for every $m \geqslant 0$, there are integers $a_{0}, a_{1}, \ldots, a_{d}$ such that

$$
h^{0}\left(V, \mathcal{O}_{V}(m)\right)=a_{d} m^{d}+a_{d-1} m^{d-1}+\ldots+a_{0}
$$

for every $m \geqslant 0$. Applying Lemma 3.3 to the polynomial $P(m)=a_{d} m^{d}+a_{d-1} m^{d-1}+\ldots+a_{0}$, we see that $a_{0}=b_{0} / c_{0}$, where $\operatorname{gcd}\left(b_{0}, c_{0}\right)=1$ and $b_{0}$ is divisible by $p$. On the other hand, applying (3.5) for $m=0$, we obtain that $a_{0}=h^{0}\left(V, \mathcal{O}_{V}\right)=1$, which is a contradiction.

\section{Five-Dimensional Singularities}

The main purpose of this section is to prove Theorem 1.16. We start with three easy observations.

Lemma 4.1. Let $\bar{G}$ be a finite subgroup in $\operatorname{Aut}\left(\mathbb{P}^{1}\right)$, and let $\Omega$ be a $\bar{G}$-orbit in $\mathbb{P}^{1}$. If $|\Omega| \in$ $\{1,2,5,7,8,9,10,11\}$, then there is a $\bar{G}$-orbit in $\mathbb{P}^{1}$ of length at most 2 .

Proof. If $|\Omega| \in\{1,2,5,7,8,9,10,11\}$, then the group $\bar{G}$ is either cyclic or dihedral, which implies that $\bar{G}$ has either a fixed point or an orbit that consists of 2 points, respectively.

Lemma 4.2. Let $\bar{G}$ be a finite subgroup in $\operatorname{Aut}\left(\mathbb{P}^{2}\right)$. Suppose that either $\bar{G} \cong \mathrm{S}_{4}$, or $\bar{G} \cong \mathrm{A}_{4}$. Then either there is a $\bar{G}$-invariant point in $\mathbb{P}^{2}$, or there exists at most one $\bar{G}$-orbit in $\mathbb{P}^{2}$ of length 3 .

Proof. If $\bar{G} \cong \mathrm{S}_{4}$, then any point fixed by the normal subgroup $\mathrm{A}_{4} \subset \bar{G}$ is also fixed by $\bar{G}$. Thus we may assume that $\bar{G} \cong \mathrm{A}_{4}$ and there exists no $\bar{G}$-invariant points in $\mathbb{P}^{2}$. Consider the unique subgroup $\Gamma \subset \bar{G} \cong \mathrm{A}_{4}$ of index 3 . If there is a $\bar{G}$-orbit $\Omega \subset \mathbb{P}^{2}$ such that $|\Omega|=3$, then $\Gamma$ is a stabilizer of any point of $\Omega$. Let $F_{\Gamma} \subset \mathbb{P}^{2}$ be the set of the points fixed by $\Gamma$. If $F_{\Gamma}$ is infinite, then there is a line $L \subset F_{\Gamma}$. Since the subgroup $\Gamma \subset \bar{G}$ is normal, the line $L$ is $\bar{G}$-invariant, and thus $\bar{G}$ fixes some point on $\mathbb{P}^{2}$ which is impossible by assumption. On the other hand, if $\Gamma$ has a finite number $r$ of fixed points, one has $r \leqslant n+1=3$.

Theorem 4.3. Let $S$ be a rational surface with at most Du Val singularities, and let $\sigma: \tilde{S} \rightarrow S$ be the minimal resolution of singularities of the surface $S$. Then

$$
\operatorname{rkPic}(\bar{S})+K_{\bar{S}}^{2}=\operatorname{rkPic}(S)+K_{S}^{2}+\sum_{P \in S} \mu(P)=10,
$$

where $\mu(P)$ is the Milnor number $]^{4}$ of the point $P$.

Proof. The required equality follows from the classical Noether formula.

Now we are ready to prove Theorem 1.16. Let $G$ be a finite subgroup in $\mathrm{GL}_{5}(\mathbb{C})$ that does not contain reflections, and let $\phi: \mathrm{GL}_{5}(\mathbb{C}) \rightarrow \mathrm{PGL}_{5}(\mathbb{C})$ be the natural projection. Put $\bar{G}=\phi(G)$. Let us identify $\mathrm{PGL}_{5}(\mathbb{C})$ with $\operatorname{Aut}\left(\mathbb{P}^{4}\right)$.

Theorem 4.4. Suppose that $G$ is transitive and does not have semi-invariants of degree at most 4 . If $\mathbb{C}^{5} / G$ is not weakly-exceptional, then there exists a $\bar{G}$-invariant irreducible nondegenerate projectively normal Fano type surface $S$ of degree 6 with at most quotient singularities that is not contained in a quadric hypersurface in $\mathbb{P}^{4}$ such that its generic hyperplane section is a projectively normal smooth curve of genus 3 .

Proof. Suppose that $\mathbb{C}^{5} / G$ is not weakly-exceptional. Then it follows from Theorem 1.12 that there is an irreducible $\bar{G}$-invariant projectively normal Fano type subvariety $S \subset \mathbb{P}^{4}$ such that

$$
\operatorname{deg}(S) \leqslant\left(\begin{array}{c}
4 \\
\operatorname{dim}(S)
\end{array}\right)
$$

\footnotetext{
${ }^{4}$ Recall that $\mu(P)=0$ if $P \notin \operatorname{Sing}(S)$, and $\mu(P)=n$ if $(S \ni P)$ is a singularity of type $\mathbb{A}_{n}$, or $\mathbb{D}_{n}$, or $\mathbb{E}_{n}$.
} 
and if $\operatorname{dim}(S) \geqslant 2$, then its general hyperplane section is also projectively normal.

Since $G$ is transitive and $S$ is $\bar{G}$-invariant, the subvariety $S$ is not contained in a hyperplane in $\mathbb{P}^{4}$. In particular, we see that $S$ is not a point. Since $G$ does not have semi-invariants of degree at most 4 , we see that $S$ is not a threefold.

If $S$ is a curve, then $\operatorname{deg}(S) \leqslant 4$, and thus $S$ is a smooth rational curve of degree 4 , because $S$ is not contained in a hyperplane in $\mathbb{P}^{4}$. On the other hand, it follows from [23, Exercise 8.7] that the secant variety of a smooth rational curve of degree 4 is a cubic hypersurface in $\mathbb{P}^{4}$. This is impossible since $G$ does not have semi-invariants of degree at most 4 and $S$ is $\bar{G}$-invariant. The obtained contradiction shows that $S$ cannot be a curve.

Thus, the subvariety $S$ is a surface. Then $S$ has at most quotient singularities by [33, Theorem 3.6]. Let $H$ be a hyperplane section of the surface $S \subset \mathbb{P}^{4}$. Then $H$ is projectively normal. Let $\mathcal{I}_{S}$ be the ideal sheaf of the surface $S$. Since $S$ is projectively normal, it follows from the Riemann-Roch theorem5 that

$$
\left(\begin{array}{c}
n+4 \\
n
\end{array}\right)-h^{0}\left(\mathcal{O}_{\mathbb{P}^{4}}(n) \otimes \mathcal{I}_{S}\right)=h^{0}\left(\mathcal{O}_{S}(n H)\right)=1+\frac{n^{2}}{2}(H \cdot H)-\frac{n}{2}\left(H \cdot K_{S}\right)
$$

for any $n \geqslant 1$. In particular, since $S$ is not contained in a hyperplane in $\mathbb{P}^{4}$, it follows from (4.5) applied for $n=1$ that $H \cdot H-H \cdot K_{S}=8$. On the other hand, we know that $H \cdot H \geqslant 3$, since $S$ is not contained in a hyperplane in $\mathbb{P}^{4}$. Moreover, since $S$ is a Fano type surface, the divisor $-K_{S}$ is big (see Definition 1.11), which implies that $-H \cdot K_{S}>0$. Thus, we see that $H \cdot H \in\{3,4,5,6,7\}$. Now plugging $n=2$ into (4.5), we see that $6-h^{0}\left(\mathcal{O}_{\mathbb{P}^{4}}(2) \otimes \mathcal{I}_{S}\right)=H \cdot H$. In particular, we must have $H \cdot H \neq 5$, since $G$ does not have semi-invariants of degree 2 . Thus, we see that $H \cdot H \in\{3,4,6\}$.

If $H \cdot H=3$, then either $S$ is a cone over a smooth rational cubic curve, or $S$ is a smooth cubic scroll (see e.g. [20]). If $S$ is a cone over a smooth rational cubic curve, then its vertex is $\bar{G}$-invariant, which is impossible since $G$ is transitive. If $S$ is a smooth cubic scroll, then there is a unique line $L \subset S$ such that $L^{2}=-1$, which implies that $L$ must be $\bar{G}$-invariant, which is again impossible, because $G$ is transitive. Thus, we see that $H \cdot H \neq 3$.

If $H \cdot H=6$, then $H \cdot K_{S}=-2$, which implies that $H$ is a smooth curve of genus 3 by the adjunction formula. Thus, if $H \cdot H=6$, then we are done, because $H \cdot H=6$ implies that $h^{0}\left(\mathcal{O}_{\mathbb{P}^{4}}(2) \otimes \mathcal{I}_{S}\right)=0$. Therefore, we may suppose that $H \cdot H=4$. Since $6-h^{0}\left(\mathcal{O}_{\mathbb{P}^{4}}(2) \otimes \mathcal{I}_{S}\right)=H \cdot H$ and $S$ is not contained in a hyperplane in $\mathbb{P}^{4}$, there are two irreducible quadrics in $\mathbb{P}^{4}$ that contain $S$. Let us denote them by $Q$ and $Q^{\prime}$. Then $S=Q \cap Q^{\prime}$, since $H \cdot H=4$.

If $S$ is singular, then $|\operatorname{Sing}(S)| \leqslant 4$, because $S$ has canonical singularities, since $S$ is a complete intersection that has Kawamata log terminal singularities. But $\operatorname{Sing}(S)$ is $\bar{G}$-invariant, which is impossible, since $G$ is transitive. Thus, the surface $S$ is smooth.

Let $\mathcal{P}$ be a pencil that is generated by the quadrics $Q$ and $Q^{\prime}$. Then $\mathcal{P}$ contains exactly 5 singular quadrics, which are simple quadric cones. Now it follows from Lemma 4.1 that there exists two quadrics in $\mathcal{P}$, say $Q_{1}$ and $Q_{2}$, such that $Q_{1}+Q_{2}$ is $\bar{G}$-invariant. The latter is impossible, because $G$ does not have semi-invariants of degree at most 4 .

To complete the proof of Theorem 1.16 we are going to show that there are no surfaces $S$ satisfying the conditions implied by Theorem 4.4. If $\mathbb{C}^{5} / G$ is weakly-exceptional, then $G$ is transitive by [46, Proposition 2.1] and does not have semi-invariants of degree at most 4 by Theorem 1.8. Thus, since Fano type surfaces are rational (cf. [58]) and have big anticanonical divisors by Definition 1.11, the assertion of Theorem 1.16 follows from Theorem 4.4 and the following

\footnotetext{
${ }^{5}$ Recall that the Riemann-Roch theorem in the usual form is valid for Cartier divisors on surfaces with quotient (and even rational) singularities, see [4, Theorem 9.1].
} 
Theorem 4.6. Let $S$ be an irreducible $\bar{G}$-invariant normal surface in $\mathbb{P}^{4}$ of degree 6 , let $H$ be its general hyperplane section. Then $-K_{S}$ is not big if the following conditions are satisfied:

(A) the surface $S$ is projectively normal,

(B) the surface $S$ is rational,

(C) the surface $S$ has at most quotient singularities,

(D) the surface $S$ is not contained in a quadric hypersurface,

(E) the curve $H$ is a smooth curve of genus 3,

(F) the curve $H$ is projectively normal (considered as a subvariety in $\mathbb{P}^{3}$ ),

(G) the group $G$ is transitive.

It should be pointed out that some of the conditions (A), (B), (C), (D), (E) and (F) in Theorem 4.6 may be redundant. For example, one can show that (F) follows from (A), (C), (D) and (E) (see the proof of [24, Theorem 2.1], [37, Theorem 4.4] and [1]). Still we prefer to keep them all, because we do not care. In the remaining part of this section we prove Theorem 4.6. To do this we will need two auxiliary results that may be useful on their own (cf. [56]).

Lemma 4.7. Let $P_{1}, \ldots, P_{10}$ be 10 different points in $\mathbb{P}^{2}$, let $\pi: \tilde{S} \rightarrow \mathbb{P}^{2}$ be the blow-up of the points $P_{1}, \ldots, P_{10}$, let $\psi: \hat{S} \rightarrow \mathbb{P}^{2}$ be the blow-up of the points $P_{1}, \ldots, P_{8}$, let $\hat{P}_{9}$ and $\hat{P}_{10}$ be the preimages of the points $P_{9}$ and $P_{10}$ on the surface $\hat{S}$. Suppose that the linear system $\left|-K_{\tilde{S}}\right|$ is empty, $-K_{\hat{S}}$ is nef and big, and neither $\hat{P}_{9}$ nor $\hat{P}_{10}$ is contained in a curve in $\hat{S}$ that has trivial intersection with $-K_{\hat{S}}$. Then $-K_{\tilde{S}}$ is not big.

Proof. The surface $\hat{S}$ is a weak del Pezzo surface, i.e. $-K_{\hat{S}}$ is big and nef. Let $\gamma: \hat{S} \rightarrow \bar{S}$ be its anticanonical model, i.e. $\gamma$ is given by the linear system $\left|-n K_{\hat{S}}\right|$ for $n \gg 0$. Then $\bar{S}$ is a del Pezzo surface with canonical singularities such that $K_{\bar{S}}^{2}=1$, and $\gamma$ contracts all curves that have trivial intersection with $-K_{\hat{S}}$ (they are $(-2)$-curves, i. e. smooth rational curves with self-intersection -2$)$. It is well-known that $\left|-2 K_{\bar{S}}\right|$ is free from base points and induces a double cover $\delta: \bar{S} \rightarrow Q$, where $Q$ is a quadric cone in $\mathbb{P}^{3}$.

By assumption both points $\gamma\left(\hat{P}_{9}\right)$ and $\gamma\left(\hat{P}_{10}\right)$ are contained in a smooth locus of the surface $\bar{S}$. Moreover, there exists no curve in $\left|-K_{\bar{S}}\right|$ that contains both points $\gamma\left(\hat{P}_{9}\right)$ and $\gamma\left(\hat{P}_{10}\right)$, because otherwise the linear system $\left|-K_{\tilde{S}}\right|$ would not be empty. Thus, the points $\delta \circ \gamma\left(\hat{P}_{9}\right)$ and $\delta \circ \gamma\left(\hat{P}_{10}\right)$ are not contained in one ruling of the quadric cone $Q$.

Let $\eta: \tilde{S} \rightarrow \hat{S}$ be the blow up of the points $\hat{P}_{9}$ and $\hat{P}_{10}$, and let $E_{9}$ and $E_{10}$ be the exceptional divisors of the blow up $\eta$ such that $\eta\left(E_{9}\right)=\hat{P}_{9}$ and $\eta\left(E_{10}\right)=\hat{P}_{10}$. Then we have a diagram

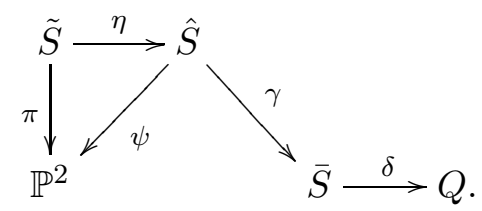

Since $-2 K_{\tilde{S}}+E_{9}+E_{10} \sim \eta^{*}\left(-2 K_{\hat{S}}\right)-E_{9}-E_{10}$, the linear system $\left|-2 K_{\tilde{S}}+E_{9}+E_{10}\right|$ does not have base curves except possibly the curves $E_{9}$ and $E_{10}$, because the points $\delta \circ \gamma\left(\hat{P}_{9}\right)$ and $\delta \circ \gamma\left(\hat{P}_{10}\right)$ are not contained in one ruling of the quadric cone $Q$. Indeed, the base locus of the linear system $\eta\left(\left|-2 K_{\tilde{S}}+E_{9}+E_{10}\right|\right)$ consists of the points of the surface $\hat{S}$ that are mapped to one of the points $\delta \circ \gamma\left(\hat{P}_{9}\right)$ or $\delta \circ \gamma\left(\hat{P}_{10}\right)$ by $\delta \circ \gamma$, because $\gamma$ is an isomorphism in a neighborhood of the points $\hat{P}_{9}$ and $\hat{P}_{10}$, and $\delta \circ \gamma\left(\hat{P}_{9}\right)$ and $\delta \circ \gamma\left(\hat{P}_{10}\right)$ are not contained in one ruling of the quadric cone $Q$. Thus, the divisor $-2 K_{\tilde{S}}+E_{9}+E_{10}$ is nef and big, because $\left(-2 K_{\tilde{S}}+E_{9}+E_{10}\right)^{2}=2$ and

$$
\left(-2 K_{\tilde{S}}+E_{9}+E_{10}\right) \cdot E_{9}=\left(-2 K_{\tilde{S}}+E_{9}+E_{10}\right) \cdot E_{10}=1 .
$$

One the other hand, we see that $-K_{\tilde{S}} \cdot\left(-2 K_{\tilde{S}}+E_{9}+E_{10}\right)=0$, which implies that $-K_{\tilde{S}}$ cannot be big. 
Lemma 4.8. Let a group $\bar{G}$ act faithfully on the projective plane $\mathbb{P}^{2}$. Let $\mathcal{P}=\left\{P_{1}, \ldots, P_{10}\right\}$ be a $\bar{G}$-invariant collection of 10 points on $\mathbb{P}^{2}$, and denote by $\pi: \tilde{S} \rightarrow \mathbb{P}^{2}$ the blow-up of the points $P_{1}, \ldots, P_{10}$. Suppose that the following conditions hold:

(i) there are no cubic curves passing through all points of $\mathcal{P}$;

(ii) if a $\bar{G}$-invariant curve $C \subset \mathbb{P}^{2}$ passes through at least $r$ of the points of $\mathcal{P}$, then one has $4 \operatorname{deg}(C)-r \geqslant 4$

(iii) there are no $\bar{G}$-orbits of length at most 2 on $\mathbb{P}^{2}$;

(iv) there are no $\bar{G}$-orbits of length at most 4 contained in $\mathbb{P}^{2} \backslash \mathcal{P}$;

Then the anticanonical class $-K_{\tilde{S}}$ is not big.

Proof. To start with, we are going to prove that the group $\bar{G}$ acts transitively on the set $\mathcal{P}$. Keeping in mind (iii), one can easily see that for a non-transitive action there are the following possibilities to split $\mathcal{P}$ into $\bar{G}$-orbits: either $\mathcal{P}$ is a union of two $\bar{G}$-orbits of length 5 , or it is a union of a $\bar{G}$-orbit of length 6 and a $\bar{G}$-orbit of length 4 , or it is a union of a $\bar{G}$-orbit of length 4 and two $\bar{G}$-orbits of length 3 , or it is a union of a $\bar{G}$-orbit of length 7 and a $\bar{G}$-orbit of length 3 . Let us exclude these possibilities case by case.

Assume that $\mathcal{P}$ contains a $\bar{G}$-orbit $\mathcal{P}^{\prime}$ of length 5 . If there are at least 3 points of $\mathcal{P}^{\prime}$ that lie on a line $L$, then each of the images of $L$ under the action of $\bar{G}$ also contains at least 3 points of $\mathcal{P}^{\prime}$. This is possible only if the $\bar{G}$-orbit of $L$ consists of at most 2 lines. The latter implies that there is a $\bar{G}$-orbit on $\mathbb{P}^{2}$ that consists of at most 2 points, which contradicts (iii). Thus, the 5 points of $\mathcal{P}^{\prime}$ are in general position, so that there is a unique conic $C$ in $\mathbb{P}^{2}$ than passing through all points of $\mathcal{P}^{\prime}$, and $C$ is non-singular. By Lemma 4.1 there is a $\bar{G}$-orbit on $C$ of length at most 2 , which contradicts (iii).

Assume that $\mathcal{P}$ contains a $\bar{G}$-orbit $\mathcal{P}^{\prime}$ of length 4 . The same argument as above shows that the points of $\mathcal{P}^{\prime}$ are in general position, so that the pencil of conics passing through the points of $\mathcal{P}^{\prime}$ has exactly 3 degenerate members, and each of them is a union of two distinct lines. The intersection points of these pairs of lines gives a $\bar{G}$-orbit $\mathcal{P}^{\prime \prime}$ of length at most 3 . By (iv) one has $\mathcal{P}^{\prime \prime} \subset \mathcal{P}$, so that by (iii) one has $\left|\mathcal{P}^{\prime \prime}\right|=3$. Thus, $\mathcal{P}^{\prime \prime \prime}=\mathcal{P} \backslash\left(\mathcal{P}^{\prime} \cup \mathcal{P}^{\prime \prime}\right)$ is another $\bar{G}$-orbit of length 3 contained in $\mathcal{P}$ by (iii).

Note that $\bar{G}$ acts faithfully on the finite set $\mathcal{P}^{\prime}$ since an automrphism of $\mathbb{P}^{2}$ is defined by the images of 4 points in general position. Hence there is an embedding $\bar{G} \hookrightarrow \mathrm{S}_{4}$. Keeping in mind that $|\bar{G}|$ is divisible by 12 since $\bar{G}$ has orbits of lengths 3 and 4 , we see that either $\bar{G} \cong \mathrm{S}_{4}$ or $\bar{G} \cong \mathrm{A}_{4}$. Lemma 4.2 implies that there is a $\bar{G}$-invariant point on $\mathbb{P}^{2}$, which is impossible by (iii).

Assume that $\mathcal{P}$ contains a $\bar{G}$-orbit $\mathcal{P}^{\prime}$ of length 7 . We are going to prove that the points of $\mathcal{P}^{\prime}$ are in general position, i. e. there are no lines passing through 3 points of $\mathcal{P}^{\prime}$ and there are no conics passing through 6 points of $\mathcal{P}^{\prime}$.

Suppose that there is a line $L_{1}$ passing through 3 points of $\mathcal{P}^{\prime}$. Let $\mathcal{L}=\left\{L_{1}, \ldots, L_{k}\right\}$ be the $\bar{G}$-orbit of the line $L$. Denote by $l$ the number of lines from $\mathcal{L}$ passing through each point from $\mathcal{P}^{\prime}$, and by $p$ the number of points from $\mathcal{P}^{\prime}$ lying on each line from $\mathcal{L}$. One has $p \geqslant 3$ by assumption and $p \leqslant 4$ by (i). On the other hand, one has $l \leqslant 3$ since $\left|\mathcal{P}^{\prime}\right|=7$. Using the equality $7 l=p k$, one finds that $k$ is divisible by 7 , and thus $l=p=3$ and $k=7$. It is easy to see that there are no irreducible conics passing through 6 of the points from $\mathcal{P}^{\prime}$ (indeed, otherwise there would exist a line from $\mathcal{L}$ intersecting such conic in at least 3 points). Let $\tilde{\Sigma}$ be a surface obtained by blowing up the 7 points of $\mathcal{P}^{\prime}$ and $\Sigma$ be an anticanonical model of $\tilde{\Sigma}$. Then $\Sigma$ is a del Pezzo surface of degree 2 with $7 \mathrm{Du}$ Val singular points of type $\mathbb{A}_{1}$ (that are images of the proper transforms of lines from $\mathcal{L}$ ), which easily leads to a contradiction.

Suppose that there is an irreducible conic $C$ passing through at least 6 points of $\mathcal{P}^{\prime}$. Then $C$ is $\bar{G}$-invariant, since otherwise there would exist another irreducible conic passing through at least 6 points of $\mathcal{P}^{\prime}$ and thus intersecting $C$ in at least 5 points. On the other hand, the curve $C$ cannot be $\bar{G}$-invariant by (ii). 
We see that the 7 points of $\mathcal{P}^{\prime}$ are in general position, so that the surface $\tilde{\Sigma}$ obtained by the blow-up $\pi^{\prime}: \tilde{\Sigma} \rightarrow \mathbb{P}^{2}$ of the points of $\mathcal{P}^{\prime}$ is a smooth del Pezzo surface of degree 2. Moreover, $\bar{G} \subset \operatorname{Aut}(\tilde{\Sigma})$, so that $|\operatorname{Aut}(\tilde{\Sigma})|$ is divisible by 7 . Using [18, Table 6], one obtains that $\bar{G}$ is a subgroup of the Klein group $\mathrm{PSL}_{2}\left(\mathbb{F}_{7}\right)$; moreover, $\bar{G}$ must be isomorphic either to $\mathrm{PSL}_{2}\left(\mathbb{F}_{7}\right)$ itself, or to a group $F_{21} \cong \mathbb{Z}_{7} \rtimes \mathbb{Z}_{3}$, or to the cyclic group $\mathbb{Z}_{7}$. The former two cases are impossible, since in $\tilde{\Sigma}$ is $\bar{G}$-minimal if $\bar{G}$ contains $F_{21}$ by [18, Theorem 6.17], which contradicts the existence of the $\bar{G}$-invariant morphism $\pi^{\prime}$. The latter case is impossible since $|\bar{G}|$ must be also divisible by 3 since $\bar{G}$ has an orbit of length 3 on $\mathbb{P}^{2}$.

We see that $\bar{G}$ acts transitively on the points of $\mathcal{P}$. Let us consider the following condition (*): there exist 8 points of $\mathcal{P}$ (say, $\left.P_{1}, \ldots, P_{8}\right)$ such that the surface $\tilde{\Sigma}$ obtained by blowing up these points is a weak del Pezzo surface, and the preimages of the other two points of $\mathcal{P}$ (i. e., $P_{9}$ and $\left.P_{10}\right)$ are not contained in $(-2)$-curves of $\tilde{\Sigma}$. Since $\left|-K_{\tilde{S}}\right|$ is empty, because there are no cubic curves passing through all points of $\mathcal{P}$, we see that it follows from Lemma 4.7 that $-K_{\tilde{S}}$ is not big assuming that $(*)$ holds. Let us show that the failure of $(*)$ leads to a contradiction.

Suppose that $(*)$ does not hold. It means that either there exists a line containing at least 4 points of $\mathcal{P}$, or there exists an irreducible conic containing at least 7 points of $\mathcal{P}$, or there exists an irreducible cubic containing at least 9 points of $\mathcal{P}$ and singular at one of these points. Moreover, (i) implies that in the latter list of possibilities one can replace "at least" by "exactly".

Suppose that there exists an irreducible cubic, say, $Z_{1}$, containing 9 points of $\mathcal{P}$, say, $P_{1}, \ldots, P_{9}$, and singular at one of these points, say, $P_{1}$. Consider an element $g_{2} \in \bar{G}$ such that $g_{2}\left(P_{1}\right)=P_{2}$ (it exists since the action of $\bar{G}$ on $\mathcal{P}$ is transitive) and put $Z_{2}=g_{2}\left(Z_{1}\right)$. Note that $Z_{2} \neq Z_{1}$ since $Z_{1}$ is smooth at $P_{2}$ while $Z_{2}$ is singular at that point. If $P_{1} \in Z_{2}$, then

$$
9=Z_{1} \cdot Z_{2} \geqslant \sum_{i=1}^{2} \operatorname{mult}_{P_{i}}\left(Z_{1}\right) \operatorname{mult}_{P_{i}}\left(Z_{2}\right)+\sum_{i=3}^{9} \operatorname{mult}_{P_{i}}\left(Z_{1}\right) \operatorname{mult}_{P_{i}}\left(Z_{2}\right)=10
$$

which is a contradiction. If $P_{1} \notin Z_{2}$, consider an element $g_{3} \in \bar{G}$ such that $g_{3}\left(P_{1}\right)=P_{3}$ and put $Z_{3}=g_{3}\left(Z_{1}\right)$. If $P_{1} \in Z_{3}$, replace $Z_{2}$ by $Z_{3}$. If $P_{1} \notin Z_{3}$, note that $P_{2} \in Z_{3}$ and $P_{3} \in Z_{2}$ and replace $Z_{1}$ by $Z_{3}$. In both cases a computation similar to (4.9) leads to a contradiction.

Suppose that there exists an irreducible conic $C_{1}$ passing through 7 points of $\mathcal{P}$. Let $\mathcal{C}=$ $\left\{C_{1}, \ldots, C_{k}\right\}$ be the $\bar{G}$-orbit of the conic $C_{1}$. Denote by $c$ the number of conics from $\mathcal{C}$ passing through each point from $\mathcal{P}$; note that there are exactly 7 points from $\mathcal{P}$ lying on each conic from $\mathcal{C}$. Thus one has $7 k=10 c$. Put $\mathcal{P}_{i}=\mathcal{P} \backslash C_{i}, 1 \leqslant i \leqslant k$. If $k \geqslant 4$, then there are indices $i_{1}, i_{2} \in\{1, \ldots, k\}$ such that $\mathcal{P}_{i_{1}} \cap \mathcal{P}_{i_{2}} \neq \varnothing$. Hence the conics $C_{i_{1}}$ and $C_{i_{2}}$ intersect by at least 5 points which is impossible. Therefore $k \leqslant 3$. Moreover, the cases $k=1$ and $k=2$ are impossible by (ii), so that $k=3$ and $10 c=21$, which is again a contradiction.

We conclude that there exists a line $L_{1}$ passing through 4 points of $\mathcal{P}$. Let $\mathcal{L}=\left\{L_{1}, \ldots, L_{k}\right\}$ be the $\bar{G}$-orbit of the line $L_{1}$. Denote by $l$ the number of lines from $\mathcal{L}$ passing through each point from $\mathcal{P}$; note that there are exactly 4 points from $\mathcal{P}$ lying on each line from $\mathcal{L}$. One has $l \leqslant 3$ since $|\mathcal{P}|=10$ and $k \geqslant 5$ by (ii). Using the equality $10 l=4 k$, one finds that $l=2$ and $k=5$. Thus, the set $\mathcal{L}$ consists of 5 lines in general position. By (ii), the group $\bar{G}$ acts transitively on the set $\mathcal{L}$. Hence there exists a $\bar{G}$-orbit $\Omega \subset \mathbb{P}^{2}$ that consists of 5 points in general position, so that there is a unique (and thus $\bar{G}$-invariant) smooth conic $C$ passing through the points of $\Omega$. By Lemma 4.1, there exists a $\bar{G}$-orbit $\Omega^{\prime} \subset C$ of length at most 2. A contradiction with (iii) completes the proof.

Now we are ready to prove Theorem 4.6. Let $S$ be an irreducible normal surface in $\mathbb{P}^{4}$ of degree 6 , let $H$ be its general hyperplane section, and let $\bar{G}$ be a finite subgroup in Aut $\left(\mathbb{P}^{5}\right)$ such that $S$ is $\bar{G}$-invariant. As usual, we identify $\operatorname{Aut}\left(\mathbb{P}^{5}\right)$ with $\mathrm{PGL}_{5}(\mathbb{C})$. Suppose that the conditions $(\mathrm{A}),(\mathrm{B}),(\mathrm{C}),(\mathrm{D}),(\mathrm{E})$ and $(\mathrm{F})$ in Theorem 4.6 are satisfied.

Lemma 4.10. The curve $H$ is not hyperelliptic. 
Proof. The required assertion follows from (F) (see [24, Theorem 2.1]).

Let $f: \tilde{S} \rightarrow S$ be the minimal resolution of singularities of the surface $S$, and let $\tilde{H}$ be the proper transform of the curve $H$ on the surface $\tilde{S}$. Then the actions of the group $\bar{G}$ lifts to the surface $\tilde{S}$, and $\tilde{H} \sim f^{*}(H)$, because $H$ is a general hyperplane section of the surface $S$. Note that $-K_{S} \cdot H=-K_{\tilde{S}} \cdot \tilde{H}=2$ by the adjunction formula and (E).

Lemma 4.11. The linear systems $\left|-K_{\tilde{S}}\right|$ and $\left|-K_{S}\right|$ are empty.

Proof. If $\left|-K_{S}\right|$ contains a $\bar{G}$-invariant fixed curve $C$, then $C$ must be either a line or a conic in $\mathbb{P}^{4}$, because $H \cdot C \leqslant-K_{S} \cdot H=2$. Hence, it follows from $(\mathrm{G})$ that $\left|-K_{S}\right|$ does not contain fixed curves if $\left|-K_{S}\right|$ is not empty.

Suppose that $\left|-K_{S}\right| \neq \varnothing$. Then every curve in $\left|-K_{S}\right|$ is either a union of two lines or a conic in $\mathbb{P}^{4}$, because $-K_{S} \cdot H=2$. Hence either $\left|-K_{S}\right|$ is free from base points or its base locus must consist of at most 4 points. The latter case is impossible by $(\mathrm{G})$. Hence, the linear system $\left|-K_{S}\right|$ is free. In particular, the divisor $-K_{S}$ is Cartier, which implies that the surface $S$ has Du Val singularities by (C). Since $-K_{S} \cdot H=2$, a generic curve in $\left|-K_{S}\right|$ must be either a disjoint union of two lines or a smooth conic, which is impossible by the adjunction formula.

Finally, $\left|-K_{\tilde{S}}\right|=\varnothing$ is implied by $\left|-K_{S}\right|=\varnothing$.

Lemma 4.12. The equalities $K_{\tilde{S}}^{2}=-1$ and $h^{0}\left(\mathcal{O}_{\tilde{S}}\left(K_{\tilde{S}}+\tilde{H}\right)\right)=3$ hold, the linear system $\left|K_{\tilde{S}}+\tilde{H}\right|$ is free from base points and induces a birational morphism $\pi: \tilde{S} \rightarrow \mathbb{P}^{2}$.

Proof. It follows from the Riemann-Roch theorem and the Nadel-Shokurov vanishing theorem that

$$
h^{0}\left(\mathcal{O}_{\tilde{S}}\left(K_{\tilde{S}}+\tilde{H}\right)\right)=\chi\left(\mathcal{O}_{\tilde{S}}\left(K_{\tilde{S}}+\tilde{H}\right)\right)=\chi\left(\mathcal{O}_{\tilde{S}}\right)+\frac{\tilde{H} \cdot\left(\tilde{H}-K_{\tilde{S}}\right)}{2}=3,
$$

because $\chi\left(\mathcal{O}_{\tilde{S}}\right)=1$ by $(\mathrm{B})$. One the other hand, there is an exact sequence of cohomology groups

$$
0 \rightarrow H^{0}\left(\mathcal{O}_{\tilde{S}}\left(K_{\tilde{S}}\right)\right) \rightarrow H^{0}\left(\mathcal{O}_{\tilde{S}}\left(K_{\tilde{S}}+\tilde{H}\right)\right) \rightarrow H^{0}\left(\mathcal{O}_{\tilde{H}}\left(K_{\tilde{S}}\right)\right)
$$

which implies that $h^{0}\left(\mathcal{O}_{\tilde{S}}\left(K_{\tilde{S}}+\tilde{H}\right)\right)=3$ and $\left|K_{\tilde{S}}+\tilde{H}\right|$ does not have base points contained in the curve $\tilde{H}$, because $h^{0}\left(\mathcal{O}_{\tilde{S}}\left(K_{\tilde{S}}\right)\right)=0$ by $(\mathrm{B}), h^{0}\left(\mathcal{O}_{\tilde{H}}\left(K_{\tilde{H}}\right)\right)=3$ by $(\mathrm{E})$, and $\left|K_{\tilde{H}}\right|$ is free from base points by (E). Thus, every base curve of the linear system $\left|K_{\tilde{S}}+\tilde{H}\right|$ is $f$-exceptional. In particular, the divisor $K_{\tilde{S}}+\tilde{H}$ is nef, since $K_{\tilde{S}}$ is $f$-nef, because the resolution of singularities $f$ is minimal. Thus, we see that

$$
K_{\tilde{S}}^{2}+2=\left(K_{\tilde{S}}+\tilde{H}\right)^{2} \geqslant 0
$$

which gives $K_{\tilde{S}}^{2} \geqslant-2$. On the other hand, it follows from the Riemann-Roch theorem that

$$
h^{0}\left(\mathcal{O}_{\tilde{S}}\left(-K_{\tilde{S}}\right)\right) \geqslant \chi\left(\mathcal{O}_{\tilde{S}}\left(-K_{\tilde{S}}\right)\right)=\chi\left(\mathcal{O}_{\tilde{S}}\right)+K_{\tilde{S}}^{2}=1+K_{\tilde{S}}^{2}
$$

because $h^{2}\left(\mathcal{O}_{\tilde{S}}\left(-K_{\tilde{S}}\right)\right)=h^{0}\left(\mathcal{O}_{\tilde{S}}\left(2 K_{\tilde{S}}\right)\right)=0$ and $\chi\left(\mathcal{O}_{\tilde{S}}\right)=1$ by $(\mathrm{B})$. Since $\left|-K_{\tilde{S}}\right|$ is empty by Lemma 4.11, we see that $K_{\tilde{S}}^{2} \leqslant-1$. Thus, either $K_{\tilde{S}}^{2}=-1$ or $K_{\tilde{S}}^{2}=-2$. Applying Theorem 4.3, we see that

$$
\operatorname{rk}(\operatorname{Pic}(\tilde{S}))=10-K_{\tilde{S}}^{2} \leqslant 12,
$$

which implies that $|\operatorname{Sing}(S)| \leqslant 11$. By $(\mathrm{G})$, we see that either $S$ is smooth, or $5 \leqslant|\operatorname{Sing}(S)| \leqslant 11$. Moreover, it follows from (4.14) and (C) and (G) that only one of the following cases is possible:

- the surface $S$ is smooth and $f$ is an isomorphism,

- $5 \leqslant|\operatorname{Sing}(S)| \leqslant 11$, and $f$ contracts exactly one smooth irreducible rational curve to every singular point of the surface $S$, 
- $|\operatorname{Sing}(S)|=5$, and $f$ contracts exactly two smooth irreducible rational curves that intersect transversally in one point to every singular point of the surface $S$.

The linear system $\left|K_{\tilde{S}}+\tilde{H}\right|$ induces a rational map $\pi: \tilde{S} \rightarrow \mathbb{P}^{2}$. If $\left|K_{\tilde{S}}+\tilde{H}\right|$ is free from base points, then it follows from (4.13) that either $K_{\tilde{S}}^{2}=-1$ and $\pi$ is a birational morphism, or $K_{\tilde{S}}^{2}=-2$ and $\left|K_{\tilde{S}}+\tilde{H}\right|$ is composed of a pencil. Moreover, if $\left|K_{\tilde{S}}+\tilde{H}\right|$ is free from base points and is composed of a pencil, then $\pi(\tilde{S})$ must be a smooth conic and $\left.\pi\right|_{\tilde{H}}: \tilde{H} \rightarrow \pi(\tilde{S})$ must be a double cover, because $\left(K_{\tilde{S}}+\tilde{H}\right) \cdot \tilde{H}=4$. By Lemma 4.10, we know that $\tilde{H}$ is not hyperelliptic. Thus, the case when $\left|K_{\tilde{S}}+\tilde{H}\right|$ is free from base points and $K_{\tilde{S}}^{2}=-2$ is impossible. Therefore, to complete the proof it is enough to prove that $\left|K_{\tilde{S}}+\tilde{H}\right|$ is free from base points.

Suppose that $\left|K_{\tilde{S}}+\tilde{H}\right|$ has a base point $P \in \tilde{S}$. Then it follows from [49, Theorem 1] that there exists an effective Cartier divisor $E$ on the surface $\tilde{S}$ such that $P \in \operatorname{Supp}(E)$ and either $\tilde{H} \cdot E=0$ and $E^{2}=-1$ or $\tilde{H} \cdot E=1$ and $E^{2}=0$.

If $\tilde{H} \cdot E=0$ and $E^{2}=-1$, then $E$ is $f$-exceptional, which implies that $S$ must be singular at the point $f(P)$. If $\tilde{H} \cdot E=1$ and $E^{2}=0$ and $S$ is smooth, then $E$ is a line in $S \subset \mathbb{P}^{4}$, which implies that $-1=K_{\tilde{S}} \cdot E+1=(E+\tilde{H}) \cdot E \geqslant 0$ by the adjunction formula, because $K_{\tilde{S}}+\tilde{H}$ is nef. Thus, in both cases the surface $S$ must be singular.

Let $r$ be the number of $f$-exceptional irreducible curves. Let us denote these curves by $E_{1}, \ldots, E_{r}$. Put $E=\tilde{L}+\sum_{i=1}^{r} a_{i} E_{i}$, where $\tilde{L}$ is an effective Cartier divisor on the surface $\tilde{S}$ such that none of its components is $f$-exceptional, and $a_{i}$ is a non-negative integer. Put $n_{i}=-E_{i}^{2}$. Then $n_{i} \geqslant 2$ for every $i \in\{1, \ldots, r\}$, because $f$ is minimal.

Suppose that $\tilde{H} \cdot E=0$ and $E^{2}=-1$. Then $E$ is $f$-exceptional, which simply means that $\tilde{L}=0$. If $f$ contracts exactly one smooth irreducible rational curve to every singular point of the surface $S$, then

$$
-2=E^{2}=\left(\sum_{i=1}^{r} a_{i} E_{i}\right)^{2}=-\sum_{i=1}^{r} a_{i}^{2} n_{i} \leqslant-2 \sum_{i=1}^{r} a_{i}^{2},
$$

which is a contradiction. Thus, we see that $|\operatorname{Sing}(S)|=5$, and $f$ contracts exactly two smooth irreducible rational curves that intersect transversally in one point to every singular point of the surface $S$. Without loss of generality, we may assume that $f\left(E_{1}\right)=f\left(E_{2}\right)=f(P)$ and $f(P) \neq f\left(E_{i}\right)$ for every $i \notin\{1,2\}$. Then

$$
-1=E^{2}=-a_{1}^{2} n_{1}+2 a_{1} b_{2}-a_{2}^{2} n_{2} \leqslant-2 a_{1}^{2}+2 a_{1} a_{2}-2 a_{2}^{2}=-a_{1}^{2}-a_{2}^{2}-\left(a_{1}+a_{2}\right)^{2},
$$

which immediately leads to a contradiction. Thus, we see that the case when $\tilde{H} \cdot E=0$ and $E^{2}=-1$ is impossible 6 .

Therefore, we see that $\tilde{H} \cdot E=1$ and $E^{2}=0$. Put $L=f(\tilde{L})$. Then $L$ is a line in $S \subset \mathbb{P}^{4}$, because $H \cdot L=\tilde{H} \cdot E=1$. We see that $\tilde{L}$ is an irreducible smooth rational curve. Then it follows from the adjunction formula that $-1-\tilde{L}^{2}=\left(K_{\tilde{S}}+\tilde{H}\right) \cdot \tilde{L} \geqslant 0$, because we already proved that the divisor $K_{\tilde{S}}+\tilde{H}$ is nef. Thus, we see that $\tilde{L}$ is a smooth rational curve on the surface $\tilde{S}$ such that $\tilde{L}^{2} \leqslant-1$. If $L \cap \operatorname{Sing}(S)=\varnothing$, then $0=E^{2}=\tilde{L}^{2}+\left(\sum_{i=1}^{r} a_{i} E_{i}\right)^{2} \leqslant \tilde{L}^{2}$, which is impossible, since $\tilde{L}^{2} \leqslant-1$. Thus, we see that $L \cap \operatorname{Sing}(S) \neq \varnothing$.

If $f$ contracts exactly one smooth irreducible rational curve to every singular point of the surface $S$, then

$$
0=E^{2} \leqslant \tilde{L}^{2}-\sum_{i=1}^{r}\left(a_{i}^{2} n_{i}-2 a_{i}\right) \leqslant \tilde{L}^{2},
$$

\footnotetext{
${ }^{6}$ As was pointed out to us by Yu. Prokhorov, the contradiction can be obtained much easier in this case. Namely, if $E^{2}=-1$ and $E$ is $f$-exceptional, then $\left(K_{\tilde{S}}+E\right) \cdot E \geqslant 0$, since $K_{\tilde{S}}$ is $f$-nef, which implies that $S$ has non-rational singularities by [4. However, the surface $S$ has rational singularities by (C). 
because either $\tilde{L} \cdot E_{i}=0$ or $\tilde{L} \cdot E_{i}=1$, since the curve $L$ is smooth (it is a line in $S \subset \mathbb{P}^{4}$ ). Keeping in mind that $\tilde{L}^{2} \leqslant-1$, we see that $|\operatorname{Sing}(S)|=5$, and $f$ contracts exactly two smooth irreducible rational curves that intersect transversally in one point to every singular point of the surface $S$. So, we have $r=10$.

Let us denote five singular points of the surface $S$ by $O_{1}, O_{2}, O_{3}, O_{4}$ and $O_{5}$. Without loss of generality, we may assume that $f\left(E_{i}\right)=f\left(E_{i+1}\right)=O_{\lceil i / 2\rceil}$ for every $i \in\{1, \ldots, 9\}$. Since $L$ is smooth and $(\mathrm{G})$ holds, we may assume that $\tilde{L} \cdot E_{1}=\tilde{L} \cdot E_{3}=\tilde{L} \cdot E_{5}=\tilde{L} \cdot E_{7}=\tilde{L} \cdot E_{9}=1$ and $\tilde{L}$ does not intersect the curves $E_{2}, E_{4}, E_{6}, E_{8}$ and $E_{10}$. Then

$$
0=E^{2}=\tilde{L}^{2}-\sum_{i=1}^{10} a_{i}^{2} n_{i}+2 \sum_{i=0}^{4} a_{2 i+1}+2 \sum_{i=0}^{4} a_{2 i+1} a_{2 i+2},
$$

which implies that $\tilde{L}^{2} \geqslant 0$. Indeed, for every $i \in\{1,3,5,7,9\}$, we have

$$
2 a_{i}+2 a_{i} a_{i+1}-a_{i}^{2} n_{i}-a_{i+1}^{2} n_{i+1} \leqslant-\left(a_{i}-a_{i+1}\right)^{2}-\left(a_{i}-1\right)^{2}-\left(a_{i+1}^{2}-1\right) \leqslant 0,
$$

because $a_{i}$ and $a_{i+1}$ are non-negative integers. Since we already proved that $\tilde{L}^{2} \leqslant-1$, we see that our assumption that $\left|K_{\tilde{S}}+\tilde{H}\right|$ has a base point was wrong, which completes the proof.

Note that the birational morphism $\pi: \tilde{S} \rightarrow \mathbb{P}^{2}$ is $\bar{G}$-equivariant, because the line bundle $K_{\tilde{S}}+\tilde{H}$ is $\bar{G}$-invariant. Since $K_{\tilde{S}}^{2}=-1$, the morphism $\pi$ contracts 10 irreducible smooth rational curves. Let us denote them by $E_{1}, E_{2}, E_{3}, E_{4}, E_{5}, E_{6}, E_{7}, E_{8}, E_{9}$ and $E_{10}$.

Lemma 4.15. The curves $E_{1}, E_{2}, E_{3}, E_{4}, E_{5}, E_{6}, E_{7}, E_{8}, E_{9}$ and $E_{10}$ are pairwise disjoint.

Proof. To complete the proof, we must show that $E_{i}^{2}=-1$ for every $i \in\{1, \ldots, 10\}$. Suppose that this is not true. Then there should be at least one curve among $E_{1}, \ldots, E_{10}$ whose selfintersection is not -1 . On the other hand, there should be at least one curve among $E_{1}, \ldots, E_{10}$ whose self-intersection is -1 . We may assume that there is $k \in\{2, \ldots, 9\}$ such that $E_{i}^{2}=-1$ for every $i \leqslant k$, and $E_{i}^{2} \neq-1$ for every $i>k$. By the adjunction formula, we have $K_{\tilde{S}} \cdot E_{i}=$ $-2-E_{i}^{2} \geqslant-1$. Since $\left(K_{\tilde{S}}+\tilde{H}\right) \cdot E_{i}=0$, we see that $E_{i}^{2} \neq-1$ if and only if $E_{i}^{2}=-2$ and $E_{i}$ is contracted by $f$ to a singular point of the surface $S$. On the other hand, if $E_{i}^{2}=-1$, then $\tilde{H} \cdot E_{i}=1$, which implies that $f\left(E_{i}\right)$ is a line in $S \subset \mathbb{P}^{4}$. In particular, we see that $E_{i}^{2}=-2$ for every $i>k$. Since the set $\cup_{i=1}^{10} E_{i}$ is $\bar{G}$-invariant, it easily follows from $(\mathrm{G})$ that $k=5$. Moreover, it follows from $(\mathrm{G})$ that $\bar{G}$ acts transitively on the set $\left\{E_{6}, E_{7}, E_{8}, E_{9}, E_{10}\right\}$.

The birational morphism $\pi$ contracts the curves $E_{1}, \ldots, E_{10}$ to 5 points in $\mathbb{P}^{2}$. Let us denote these points by $\mathrm{O}_{1}, \mathrm{O}_{2}, \mathrm{O}_{3}, \mathrm{O}_{4}$ and $\mathrm{O}_{5}$. Without loss of generality, we may assume that $E_{i}$ and $E_{i+5}$ are contracted by $f$ to the point $O_{i}$ for every $i \in\{1, \ldots, 5\}$.

Let $L$ be a line in $\mathbb{P}^{2}$, and let $\tilde{L}$ be its proper transform on the surface $\tilde{S}$. Then it follows from the adjunction formula that $\tilde{H} \cdot \tilde{L}=3+\tilde{L}^{2}$, which implies that $\tilde{L}^{2} \geqslant-3$, and $\tilde{L}^{2}=-3$ if and only if $\tilde{L}$ is contracted by $f$ to a singular point of the surface $S$. In particular, we see that there exists no line in $\mathbb{P}^{2}$ that passes through all points $O_{1}, \ldots, O_{5}$. Similarly, if $L$ contains four points among $O_{1}, \ldots, O_{5}$, then $L$ must be $\bar{G}$-invariant and $\tilde{L}^{2} \leqslant-3$, which implies that $f(\tilde{L})$ is a $\bar{G}$-invariant point in $S$, which is impossible by $(\mathrm{G})$.

We see that no 4 among the points $O_{1}, O_{2}, O_{3}, O_{4}$ and $O_{5}$ lie on a line in $\mathbb{P}^{2}$. In particular, there exists unique reduced conic in $\mathbb{P}^{2}$ that passes through the points $O_{1}, O_{2}, O_{3}, O_{4}$ and $O_{5}$. Let us denote this conic by $C$, and let us denote by $\tilde{C}$ its proper transform on the surface $\tilde{S}$. Then $C$ and $\tilde{C}$ are $\bar{G}$-invariant curves. If $C$ is irreducible, then $\tilde{C}^{2} \leqslant-1$ and it follows from the adjunction formula that $\tilde{H} \cdot \tilde{C}=4+\tilde{C}^{2} \leqslant 3$, which implies that $f(\tilde{C})$ is contained in a proper $\bar{G}$-invariant linear subspace in $\mathbb{P}^{4}$, which is impossible by $(\mathrm{G})$. Thus, the conic $C$ is reducible. Then at least one component of the curve $f(\tilde{C})$ (not necessary the linear span of this component) is contained in a proper $\bar{G}$-invariant linear subspace in $\mathbb{P}^{4}$, which is impossible by $(\mathrm{G})$. 
Put $P_{i}=\pi\left(E_{i}\right), 1 \leqslant i \leqslant 10$, and put $\mathcal{P}=\left\{P_{1}, P_{2}, \ldots, P_{10}\right\}$. Let us check that $\mathcal{P}$ satisfies all hypothesis of Lemma 4.8. It follows from Lemma 4.11 that there are no cubic curves passing through all points of $\mathcal{P}$.

Lemma 4.16. Let $C$ be a $\bar{G}$-invariant curve in $\mathbb{P}^{2}$ that passes through at least $r$ of the points of $\mathcal{P}$. Then $4 \operatorname{deg}(C)-r \geqslant 4$.

Proof. Suppose that $4 \operatorname{deg}(C)-r \leqslant 3$. Let us show that this assumption leads to a contradiction.

Let $\tilde{C}$ be the proper transform of the curve $C$ on the surface $\tilde{S}$. Then $\tilde{C}$ is $\bar{G}$-invariant, which implies that the set $f(\tilde{C})$ is also $\bar{G}$-invariant.

Since $\operatorname{deg}(C) \leqslant 3 / 4+r / 4 \leqslant 3 / 4+10 / 4=13 / 4$, we see that $\operatorname{deg} C \leqslant 3$. Hence $C$ consists of at most three irreducible components. In particular, it follows from $(\mathrm{G})$ that no components of the curve $\tilde{C}$ are contracted by $f$. Hence, we see that $f(\tilde{C})$ is a curve in $S \subset \mathbb{P}^{4}$ of degree

$$
\begin{aligned}
& f^{*}(H) \cdot \tilde{C}=\left(\pi^{*}\left(\mathcal{O}_{\mathbb{P}^{2}}(4)\right)-\sum_{i=1}^{10} E_{i}\right) \\
& \quad=4 \operatorname{deg}(C)-\sum_{i=1}^{10} \operatorname{mult}_{P_{i}}(C) \leqslant 4 \operatorname{deg}(C)-r \leqslant 3,
\end{aligned}
$$

which immediately implies that $\tilde{C}$ is reducible by $(\mathrm{G})$, because irreducible curve of degree at most 3 is contained in a hyperplane in $\mathbb{P}^{4}$. Moreover, we see that $f(\tilde{C})$ is a union of three different lines by $(\mathrm{G})$, because any curve of degree at most 2 is contained in a hyperplane in $\mathbb{P}^{4}$. Since $\operatorname{deg} C \leqslant 3$, we see that $C$ is a union of three lines. Then it follows from (4.17) that

$$
3=f^{*}(H) \cdot \tilde{C}=12-\sum_{i=1}^{10} \operatorname{mult}_{P_{i}}(C) \leqslant 12-r \leqslant 3,
$$

which implies that $r=9$. Thus, the curve $C$ passes through exactly 9 points in $\mathcal{P}$, which implies that (at least) one curve among $f\left(E_{i}\right), 1 \leqslant i \leqslant 10$, is $\bar{G}$-invariant, which is impossible by (G), because the curves $f\left(E_{i}\right)$ are lines in $S \subset \mathbb{P}^{4}$.

It follows from (G) that there are no $\bar{G}$-orbits of length at most 4 contained in $S$. Thus, there are no $\bar{G}$-orbits of length at most 4 contained in $\tilde{S}$, which implies that there are no $\bar{G}$-orbits of length at most 4 contained in $\mathbb{P}^{2} \backslash \mathcal{P}$. Similarly, it follows from $(\mathrm{G})$ that there are no $\bar{G}$-orbits of length at most 2 on $\mathcal{P}$, because the curves $f\left(E_{i}\right)$ are lines in $S \subset \mathbb{P}^{4}$. Therefore, we see that $\mathcal{P}$ satisfies all hypothesis of Lemma 4.8. Thus, the divisor $-K_{\tilde{S}}$ is not big by Lemma 4.8, which completes the proof of Theorem 4.6 .

\section{SiX-Dimensional SINGULARITIES}

The main purpose of this section is to prove Theorem 1.17. We start with an easy observation.

Lemma 5.1. Let $G$ be a transitive finite subgroup in $\mathrm{GL}_{n+1}(\mathbb{C})$ such that $\bar{G} \cong \mathrm{S}_{4}$. Then $n \leqslant 3$.

Proof. Since the Schur multiplier of the group $\bar{G} \cong \mathrm{S}_{4}$ is $\mathbb{Z}_{2}$, we may assume that either $G \cong \mathrm{S}_{4}$ or $G \cong 2 . \mathrm{S}_{4}$. Therefore, there is an irreducible $(n+1)$-dimensional linear representation $V \cong \mathbb{C}^{n+1}$ of either the group $2 . \mathrm{S}_{4}$, or the group $\mathrm{S}_{4}$. Since the group $\mathrm{S}_{4}$ does not have irreducible representations of dimension greater than 4 , we may assume that $G \cong 2 . \mathrm{S}_{4}$ and the center of the group $2 . \mathrm{S}_{4}$ acts non-trivially on $V$. Then $(n+1)^{2} \leqslant\left|2 . \mathrm{S}_{4}\right|-\left|\mathrm{S}_{4}\right|=24$, which implies that $n \leqslant 3$.

Now we are ready to prove Theorem 1.17. Let $G$ be a finite subgroup in $\mathrm{GL}_{6}(\mathbb{C})$ that does not contain reflections, and let $\phi: \mathrm{GL}_{6}(\mathbb{C}) \rightarrow \mathrm{PGL}_{6}(\mathbb{C})$ be the natural projection. Put $\bar{G}=\phi(G)$. Let us identify $\mathrm{PGL}_{6}(\mathbb{C})$ with $\operatorname{Aut}\left(\mathbb{P}^{5}\right)$. Suppose that $G$ is transitive, the group $G$ does not have semi-invariants of degree at most 5 , there is no irreducible $\bar{G}$-invariant smooth rational 
cubic scroll in $\mathbb{P}^{5}$, and there is no irreducible $\bar{G}$-invariant complete intersection of two quadric hypersurfaces in $\mathbb{P}^{5}$.

Theorem 5.2. If $\mathbb{C}^{6} / G$ is not weakly-exceptional, then there exists an irreducible $\bar{G}$-invariant, normal, projectively normal, non-degenerate Fano type threefold $X \subset \mathbb{P}^{5}$ of degree 6 and sectional genus 3 such that $h^{0}\left(\mathcal{O}_{\mathbb{P}^{5}}(2) \otimes \mathcal{I}_{X}\right)=0$ and $h^{0}\left(\mathcal{O}_{\mathbb{P}^{5}}(3) \otimes \mathcal{I}_{X}\right)=4$.

In the remaining part of this section we will prove Theorem [5.2, which implies Theorem 1.17. Suppose that $\mathbb{C}^{6} / G$ is not weakly-exceptional. By Theorem 1.12, there is an irreducible $\bar{G}$ invariant, irreducible, normal, projectively normal Fano type subvariety $X \subset \mathbb{P}^{5}$ such that

$$
\operatorname{deg}(X) \leqslant\left(\begin{array}{c}
5 \\
\operatorname{dim}(X)
\end{array}\right)
$$

and $X$ has more additional properties that we are about to describe. Let $\mathcal{I}_{X}$ be the ideal sheaf of the subvariety $X \subset \mathbb{P}^{5}$. Then $h^{i}\left(\mathcal{O}_{\mathbb{P}^{5}}(m) \otimes \mathcal{I}_{X}\right)=0$ for every $i \geqslant 1$ and for every $m \geqslant 0$. Let $H$ be a general hyperplane section of the subvariety $X \subset \mathbb{P}^{5}$. If $\operatorname{dim}(X) \geqslant 2$, then $H$ is irreducible, projectively normal and $h^{i}\left(\mathcal{O}_{H} \otimes \mathcal{O}_{\mathbb{P}^{5}}(m)\right)=0$ for every $i \geqslant 1$ and $m \geqslant 1$. Since $G$ is transitive and $X$ is $\bar{G}$-invariant, the subvariety $X$ is not contained in a hyperplane in $\mathbb{P}^{5}$. In particular, we see that $X$ is not a point. Since $G$ does not have semi-invariants of degree at most 5 , we see that $X$ is not a fourfold.

Lemma 5.3. The linear system $|m H|$ does not have $\bar{G}$-invariant divisors for every positive integer $m \leqslant 5$.

Proof. Since $X$ is projectively normal, the sequence

$$
0 \longrightarrow H^{0}\left(\mathcal{O}_{\mathbb{P}^{5}}(m) \otimes \mathcal{I}_{X}\right) \longrightarrow H^{0}\left(\mathcal{O}_{\mathbb{P}^{5}}(m)\right) \longrightarrow H^{0}\left(\mathcal{O}_{X}(m H)\right) \longrightarrow 0
$$

is exact. We can consider (5.4) as an exact sequence of linear $G$-representations. If $|m H|$ contains a $\bar{G}$-invariant divisor, then $G$ has a semi-invariant of degree $m$. But $G$ does not have semi-invariants of degree at most 5 by assumption.

Unfortunately, we can not claim now that $X$ has Kawamata log terminal singularities, since $-K_{X}$ is not necessary a Cartier divisor. Nevertheless, we know that $X$ has at most rational singularities (see [32, Theorem 1.3.6]). If $X$ is a surface, then $X$ has quotient singularities.

Lemma 5.5. The subvariety $X$ is not a curve.

Proof. If $X$ is a curve, then $X$ is a smooth rational curve of degree 5 , because $\operatorname{deg}(X) \leqslant 5$ and $X$ is not contained in a hyperplane in $\mathbb{P}^{5}$. Then $\bar{G}$ acts faithfully on $X$. If $\bar{G} \neq \mathrm{S}_{4}$, then there is a $\bar{G}$-invariant effective divisor in $\operatorname{Pic}\left(\mathbb{P}^{1}\right)$ of degree 20 , which is impossible by Lemma 5.3 . Since the group $G$ does not have semi-invariants of degree at most 5 , we see that $\bar{G} \cong \mathrm{S}_{4}$, which contradicts Lemma 5.1, because $G$ is transitive.

Lemma 5.6. If $h^{0}\left(\mathcal{O}_{\mathbb{P}^{5}}(2) \otimes \mathcal{I}_{X}\right) \neq 0$, then $h^{0}\left(\mathcal{O}_{\mathbb{P}^{5}}(2) \otimes \mathcal{I}_{X}\right) \geqslant 3$.

Proof. Since the group $G$ does not have semi-invariants of degree at most 5 , we see that $h^{0}\left(\mathcal{O}_{\mathbb{P}^{5}}(2) \otimes \mathcal{I}_{X}\right) \neq 1$. Thus, we must prove that $h^{0}\left(\mathcal{O}_{\mathbb{P}^{5}}(2) \otimes \mathcal{I}_{X}\right) \neq 2$.

Suppose that $h^{0}\left(\mathcal{O}_{\mathbb{P}^{5}}(2) \otimes \mathcal{I}_{X}\right)=2$. Then there exists a $\bar{G}$-invariant pencil of quadrics in $\mathbb{P}^{5}$ whose base locus contains $X$. Let us denote this pencil by $\mathcal{P}$. Since the group $G$ does not have semi-invariants of degree at most 5 and $G$ is transitive, the base locus of the pencil $\mathcal{P}$ is an irreducible threefold that is a $\bar{G}$-invariant complete intersection of two quadrics in $\mathcal{P}$, which is impossible by assumption.

Since $X$ is projectively normal, there is an exact sequence of cohomology groups

$$
0 \longrightarrow H^{0}\left(\mathcal{O}_{\mathbb{P}^{5}}(n) \otimes \mathcal{I}_{X}\right) \longrightarrow H^{0}\left(\mathcal{O}_{\mathbb{P}^{5}}(n)\right) \longrightarrow H^{0}\left(\mathcal{O}_{X}(n H)\right) \longrightarrow 0
$$


for every $n \geqslant 1$. Let $f: \tilde{X} \rightarrow X$ be the resolution of singularities of the variety $X$. If $X$ is a surface, then we assume that $f$ is a minimal resolution of singularities. Put $\tilde{H}=f^{*}(H)$. Then $\tilde{H}$ is nef and big. Hence, it follows from the Nadel-Shokurov vanishing theorem that

$$
\chi\left(\mathcal{O}_{\tilde{X}}\left(K_{\tilde{X}}+\tilde{H}\right)\right)=h^{0}\left(\mathcal{O}_{\tilde{X}}\left(K_{\tilde{X}}+\tilde{H}\right)\right) .
$$

Lemma 5.9. If $X$ is a surface, then $\operatorname{deg}(X) \neq 4$.

Proof. Suppose that $X$ is a surface and $\operatorname{deg}(X)=4$. Let us use the usual notation for the rational normal scrolls (see e.g. [23, Example 8.2.6]). Since $X$ is non-degenerate, it follows from [20] that $X$ is either a cone over a rational normal curve in $\mathbb{P}^{4}$, or a rational normal scroll $X_{1,3}$, or a rational normal scroll $X_{2,2}$, or a Veronese surface. The first two cases are impossible since otherwise there would exist a $\bar{G}$-invariant point or a $\bar{G}$-invariant line in $\mathbb{P}^{5}$, respectively, which would contradict the transitivity of $G$. If $X$ is a rational normal scroll $X_{2,2}$, then the family of linear spans of the one-parameter family of conics on $X$ sweeps out an irreducible $\bar{G}$-invariant smooth rational cubic scroll, which is impossible, since we assume that there is no such threefolds in $\mathbb{P}^{5}$. Thus, we see that $X$ is a Veronese surface. Then it follows from [23, Exercise 8.8] that the secant variety of the surface $X$ is a cubic hypersurface in $\mathbb{P}^{5}$, which must be $\bar{G}$-invariant, because $X$ is $\bar{G}$-invariant. The latter is impossible, since we assume that the group $G$ does not have semi-invariants of degree at most 5 .

Lemma 5.10. If $X$ is a surface and $\operatorname{deg}(X)=5$, then $-K_{X} \cdot H \neq 5$.

Proof. Suppose that $X$ is a surface such that $\operatorname{deg}(X)=5$ and $-K_{X} \cdot H=5$. It follows from (5.8) and the Riemann-Roch theorem that

$$
h^{0}\left(\mathcal{O}_{\tilde{X}}\left(K_{\tilde{X}}+\tilde{H}\right)\right)=1+\frac{\left(K_{\tilde{X}}+\tilde{H}\right) \cdot \tilde{H}}{2}=1,
$$

which means that there is a unique effective divisor $D$ in the linear system $\left|K_{\tilde{X}}+\tilde{H}\right|$. Moreover, since $D \cdot \tilde{H}=0$, the support of the divisor $D$ is contained in the exceptional locus of the morphism $f$. On the other hand, for any effective divisor $E$ supported in the exceptional locus of the morphism $f$, one has $K_{\tilde{X}} \cdot E \geqslant 0$ and $E \cdot \tilde{H}=0$, because we assume that $f$ is a minimal resolution of singularities. Therefore, we must have $D \sim 0$. Since, $K_{X}+H \sim f_{*}\left(K_{\tilde{X}}+\tilde{H}\right)$, we see that $K_{\tilde{X}} \sim-\tilde{H} \sim f^{*}\left(K_{X}\right)$, which implies that $X$ is a del Pezzo surface with $K_{X}^{2}=5$ that has at most Du Val singularities. Then $X$ cannot have more than 4 singular points, which implies that $X$ is smooth, because the group $G$ is transitive. We see that $X$ is an anticanonically embedded smooth del Pezzo surface of degree 5. Thus the action of $\bar{G}$ lifts isomorphically to the vector space $H^{0}\left(\mathcal{O}_{X}(H)\right)=H^{0}\left(\mathcal{O}_{X}\left(-K_{X}\right)\right) \cong \mathbb{C}^{6}$. Moreover, it follows from the transitivity of the group $G$ that $\bar{G}$ acts faithfully on the surface $X$. Thus, the group $\bar{G}$ is a subgroup of the symmetric group $\mathrm{S}_{5}$ acting on $\mathbb{C}^{6}$, since $\operatorname{Aut}(X) \cong \mathrm{S}_{5}$. The sum of all $(-1)$-curves in $X$ is a $\bar{G}$-invariant divisor that is linearly equivalent to $-2 K_{X}$ (cf. [8, Lemma 5.7]), which is impossible by Lemma 5.3 .

Lemma 5.11. If $X$ is a surface and $\operatorname{deg}(X)=6$, then $-K_{X} \cdot H \neq 4$.

Proof. Suppose that $X$ is a surface such that $\operatorname{deg}(X)=6$ and $-K_{X} \cdot H=4$. It follows from (5.8) and the Riemann-Roch theorem that

$$
h^{0}\left(\mathcal{O}_{\tilde{X}}\left(K_{\tilde{X}}+\tilde{H}\right)\right)=1+\frac{\left(K_{\tilde{X}}+\tilde{H}\right) \cdot \tilde{H}}{2}=2 .
$$

Let $\tilde{C}_{1}$ and $\tilde{C}_{2}$ be general curves in $\left|K_{\tilde{X}}+\tilde{H}\right|$. Put $C_{1}=f\left(\tilde{C}_{1}\right)$ and $C_{2}=f\left(\tilde{C}_{2}\right)$. Then every curve in the pencil $\left|K_{X}+H\right|$ is a curve of degree at most 2 , since $H \cdot C_{1}=H \cdot C_{2}=2$. In particular, either the base locus of the linear system $\left|K_{X}+H\right|$ contains a $\bar{G}$-invariant curve of degree at most 2 , or the intersection $C_{1} \cap C_{2}$ consists of at most 4 points. Since $G$ is 
transitive, we must have $C_{1} \cap C_{2}=\varnothing$, so that $\left|K_{X}+H\right|$ is base point free. In particular, the divisor $K_{X}$ is Cartier, which implies that $X$ has at most Du Val singularities. Furthermore, one has $\left(K_{X}+H\right)^{2}=0$, which implies that $K_{X}^{2}=2$, and the pencil $\left|K_{X}+H\right|$ induces a morphism $\vartheta: X \rightarrow \mathbb{P}^{1}$ whose general fiber is a smooth rational curve. Then the morphism $\vartheta$ induces a homomorphism $\theta: \bar{G} \rightarrow \operatorname{Aut}\left(\mathbb{P}^{1}\right)$.

Note that $h^{2}\left(\mathcal{O}_{X}\left(-K_{X}\right)\right)=h^{0}\left(\mathcal{O}_{X}\left(2 K_{X}\right)\right)$ by the Serre duality, and $h^{0}\left(\mathcal{O}_{X}\left(2 K_{X}\right)\right)=0$ since $H \cdot K_{X}<0$. Then $h^{0}\left(\mathcal{O}_{X}\left(-K_{X}\right)\right) \geqslant 1+K_{X}^{2}=3$ by the Riemann-Roch theorem. Let us show that the base locus of the linear system $\left|-K_{X}\right|$ does not contain curves.

Suppose that the base locus of the linear system $\left|-K_{X}\right|$ contains curves. Then the base locus of the linear system $\left|-K_{X}\right|$ contains a $\bar{G}$-invariant curve. Let us denote it by $E$. Then $\operatorname{deg}(E) \leqslant 3$, because $\operatorname{deg}(E) \leqslant-K_{X} \cdot H=4$ and $h^{0}\left(\mathcal{O}_{X}\left(-K_{X}\right)\right)>1$. Since $G$ is transitive, the only possibility is that $E$ is a disjoint union of three lines in $\mathbb{P}^{5}$. Let us denote these lines by $L_{1}, L_{2}$, and $L_{3}$. Then $G$ acts transitively on the set $\left\{L_{1}, L_{2}, L_{3}\right\}$. Moreover, there is a line $L$ in $X \subset \mathbb{P}^{5}$ such that $-K_{X} \sim L_{1}+L_{2}+L_{3}+L$, and $|L|$ is $\bar{G}$-invariant and free from base curves. Applying the adjunction formula to $L$, we see that $-2=\left(K_{X}+L\right) \cdot L=-3 L_{1} \cdot L$, which is a contradiction. Hence, the base locus of the linear system $\left|-K_{X}\right|$ does not contain curves.

Since the base locus of the linear system $\left|-K_{X}\right|$ does not contain curves, the divisor $-K_{X}$ is nef. Furthermore, the divisor $-K_{X}$ is big since $\left(-K_{X}\right)^{2}=2$, which implies that $X$ is a weak del Pezzo surface of degree 2. Let $\sigma: X \rightarrow \bar{X}$ be the blow-down of all curves that has trivial intersection with $-K_{X}$. Then $\bar{X}$ is a possibly singular del Pezzo surface of degree 2, i.e. $-K_{\bar{X}}$ is ample and $K_{\bar{X}}^{2}=2$. Moreover, the action of the group $\bar{G}$ on the surface $X$ induces a faithful action of the group $\bar{G}$ on the surface $\bar{X}$.

Let $\kappa: \bar{X} \rightarrow \mathbb{P}^{2}$ be the double cover that is given by the linear system $\left|-K_{\bar{X}}\right|$, let $R$ be the ramification divisor in $\mathbb{P}^{2}$ of the double cover $\kappa$, let $\bar{R}$ be the curve in $\bar{X}$ such that $\kappa(\bar{R})=R$, and let $\xi$ be the involution in $\operatorname{Aut}(\bar{X})$ that is induced by $\kappa$. Then $\xi$ induces an exact sequence of groups

$$
1 \longrightarrow \mathbb{Z}_{2} \longrightarrow \text { Aut }(\bar{X}) \stackrel{\beta}{\longrightarrow} \Gamma \longrightarrow
$$

where $\operatorname{im}(\alpha)=\langle\xi\rangle, \Gamma$ is a subgroup in $\operatorname{Aut}\left(\mathbb{P}^{2}\right)$ such that $R$ is $\Gamma$-invariant, and $\beta$ is induced by the double cover $\kappa$. Since $\bar{X}$ is a quartic hypersurface in $\mathbb{P}(1,1,1,2)$ and $\kappa$ is induced by the natural projection $\mathbb{P}(1,1,1,2) \rightarrow \mathbb{P}^{2}$, this sequence splits, so we have $\operatorname{Aut}(\bar{X}) \cong \Gamma \times \mathbb{Z}_{2}$. Note that $\xi$ is known as the Geiser involution of the surface $\bar{X}$.

Suppose that $X$ is singular. Then it follows from Theorem 4.3 that

$$
\operatorname{rkPic}(X)+K_{X}^{2}+\sum_{P \in X} \mu(P)=10
$$

where $\mu(P)$ is a Milnor number of a point $P \in X$. Since $G$ is transitive, we see that $|\operatorname{Sing}(X)| \geqslant 6$, and all singular points of the surface $X$ are ordinary double points. By (5.12) one has $|\operatorname{Sing}(X)| \leqslant 7$. If $|\operatorname{Sing}(X)|=7$, then $\operatorname{rkPic}(X)=1$, which implies that $X \cong \bar{X}$ is a del Pezzo surface of degree 2 that has 7 singular points, which easily leads to a contradiction. But $G$ acts transitively on the set of singular points of the surface $X$ (indeed, otherwise $\bar{G}$ would have an orbit of length at most 3 , which is impossible since $G$ is transitive). Thus, we see that $|\operatorname{Sing}(X)|=6$, which implies that either $-K_{X}$ is already ample, or there exists an irreducible curve $Z \subset X$ such that $-K_{X} \cdot Z=0$. In the latter case $\operatorname{Sing}(X) \cap C \neq \varnothing$, since $\bar{X}$ can not have more than 6 singular points. Thus, if there exists an irreducible curve $Z \subset X$ such that $-K_{X} \cdot Z=0$, then $Z \cong \mathbb{P}^{1}$ and $Z$ contains all singular points of the surface $X$. Applying the adjunction formula to the proper transform of the curve $Z$ on the surface $\tilde{X}$, we see that $Z^{2}=1$, which is impossible, since $Z^{2}<0$, because $Z$ is contracted by $\sigma$. Thus, we see that $-K_{X}$ is ample, so that $X=\bar{X}$ is a del Pezzo surface of degree 2 with 6 singular points. In fact, such del Pezzo surface is unique, since $R$ must be a union of 4 lines in general position. Since 
$\operatorname{rkPic}(X)=2$ by (5.12), every fiber of the morphism $\vartheta$ is an irreducible rational curve. Since $X$ has only ordinary double points, every fiber of $\vartheta$ contains either none or two singular points of the surface $X$. Thus, six singular points of the surface $X$ must split in pairs, which implies that there is a $\theta(\bar{G})$-orbit in $\mathbb{P}^{1}$ that contains at most 3 points. Then there is a $\theta(\bar{G})$-orbit in $\mathbb{P}^{1}$ that contains at most 2 points. In particular, there exist two fibres $F_{1}$ and $F_{2}$ of the morphism $\vartheta$ such that the divisor $F_{1}+F_{2}$ is $\bar{G}$-invariant. Hence $F_{1}+F_{2}+\bar{R}$ is a $\bar{G}$-invariant divisor as well, but $F_{1}+F_{2}+\bar{R} \sim 2 H$, which is impossible by Lemma 5.3. The obtained contradiction shows that the surface $X$ can not be singular.

Therefore, the surface $X$ is smooth. Then every fiber of the morphism $\vartheta$ is reduced, and $\vartheta$ has exactly 6 reducible fibers, which consist of two smooth rational curves intersecting transversally in one point. In particular, we see that there exists a $\bar{G}$-orbit in $X$ that consists of 6 points, since there are no $\bar{G}$-orbits in $X$ that consist of at most 5 points, because $G$ is transitive. Then there exists a $\theta(\bar{G})$-orbit in $\mathbb{P}^{1}$ that consists of 6 points, which implies that $\theta(\bar{G}) \not \mathrm{A}_{5}$. In particular, we see that $\bar{G} ¥ \mathrm{~A}_{5}$. We must consider two cases: $X \cong \bar{X}$ and $X \not \bar{X}$.

Suppose that $X \cong \bar{X}$ and $\theta(\bar{G}) \nsucceq \mathrm{S}_{4}$. Then there exists a $\theta(\bar{G})$-orbit in $\mathbb{P}^{1}$ that contains either 1 or 2 or 4 points, because we already proved that $\theta(\bar{G}) \neq \mathrm{A}_{5}$. Thus, there are 4 (not necessary distinct) fibers $\bar{F}_{1}, \bar{F}_{2}, \bar{F}_{3}, \bar{F}_{4}$ of the morphism $\vartheta$ such that the divisor $\sum_{i=1}^{4} \bar{F}_{i}$ is $\bar{G}$-invariant. Then $2 \bar{R}+\sum_{i=1}^{4} \bar{F}_{i} \sim 4 H$, and the divisor $2 \bar{R}+\sum_{i=1}^{4} \bar{F}_{i}$ is $\bar{G}$-invariant, which is impossible by Lemma 5.3. The obtained contradiction shows that $\theta(\bar{G}) \cong \mathrm{S}_{4}$ if $X \cong \bar{X}$.

Suppose that $X \cong \bar{X}$. Then $\theta(\bar{G}) \cong \mathrm{S}_{4}$. Moreover, it follows from Lemma 5.1 that $\bar{G} ¥ \mathrm{~S}_{4}$, which implies that $|\bar{G}| \geqslant 48$. Since $X /\langle\xi\rangle \cong \mathbb{P}^{2}$, we see that $\langle\xi\rangle$-invariant subgroup of the group $\operatorname{Pic}(X)$ is $\mathbb{Z}$, which implies that $\xi \notin \bar{G}$, since the pencil $\left|K_{X}+H\right|$ is $\bar{G}$-invariant. Thus, we see that $\bar{G} \cong \beta(\bar{G})$. Browsing through the list of the possible automorphism groups of smooth del Pezzo surfaces of degree 2 (see [18, Table 6]) and using the fact that $|\bar{G}|$ is divisible by 24 and $|\bar{G}| \geqslant 48$, we see that $\Gamma$ is a subgroup of the following groups: $\operatorname{PSL}_{2}\left(\mathbb{F}_{7}\right),\left(\mathbb{Z}_{4} \times \mathbb{Z}_{4}\right) \rtimes \mathrm{S}_{3}$, or $4 . \mathrm{A}_{4}$. On the other hand, the only subgroup of the group $\mathrm{PSL}_{2}\left(\mathbb{F}_{7}\right)$ that admits a surjective homomorphism to $\mathrm{S}_{4}$ is isomorphic to $\mathrm{S}_{4}$ (see [14]), which implies that either $\Gamma \cong\left(\mathbb{Z}_{4} \times \mathbb{Z}_{4}\right) \rtimes \mathrm{S}_{3}$ or $\Gamma \cong 4$. $\mathrm{A}_{4}$. If $\Gamma \cong 4 . \mathrm{A}_{4}$, then the center of the group $\bar{G}$ must be contained in the kernel of the homomorphism $\psi$, because the group $\mathrm{S}_{4}$ has trivial center. Thus, if $\Gamma \cong 4 . \mathrm{A}_{4}$, then the monomorphism $\beta$ composed with the natural epimorphism $4 . \mathrm{A}_{4} \rightarrow \mathrm{A}_{4}$ gives a monomorphism $\bar{G} \rightarrow \mathrm{A}_{4}$, which is impossible, since $|\bar{G}| \geqslant 48$. Thus, we see that $\Gamma \cong\left(\mathbb{Z}_{4} \times \mathbb{Z}_{4}\right) \rtimes \mathrm{S}_{3}$. Then it follows from the existence of the epimorphism $\psi: \bar{G} \rightarrow \mathrm{S}_{4}$, that either $\bar{G} \cong \beta(\bar{G})=\Gamma \cong\left(\mathbb{Z}_{4} \times \mathbb{Z}_{4}\right) \rtimes \mathrm{S}_{3}$, or $\Gamma \cong \mathrm{S}_{4}$ (cf. the proof of [18, Theorem 6.17]). Since we already know that $\Gamma \neq \mathrm{S}_{4}$, then $\bar{G} \cong \Gamma$. It follows from the proof of [18, Theorem 6.17] that the $\bar{G}$-invariant subgroup of the Picard group is $\mathbb{Z}$, which is a contradiction, since $\left|K_{X}+H\right|$ is $\bar{G}$-invariant. The obtained contradiction shows that $X \neq \bar{X}$.

Since $X \not \bar{X}$, we see that the divisor $-K_{X}$ is not ample. Let $E_{1}, \ldots, E_{r}$ be all irreducible curves that are contracted by $\sigma$, let $C_{1}$ and $C_{2}$ be general curves in the pencil $\left|K_{X}+H\right|$. Then $C_{1}$ and $C_{2}$ are smooth irreducible curves, and $R_{i} \cdot E_{j} \geqslant 1$ for all $i$ and $j$, since none of the curves $E_{1}, \ldots, E_{r}$ can not be a component of any curve in the pencil $\left|K_{X}+H\right|$. Since

$$
-K_{\bar{X}} \cdot \sigma\left(C_{1}\right)=-K_{X} \cdot C_{1}=-K_{\bar{X}} \cdot \sigma\left(C_{2}\right)=-K_{X} \cdot C_{2}=2,
$$

we see that either the curves $\sigma\left(C_{1}\right)$ and $\sigma\left(C_{2}\right)$ are smooth rational curves such that $\kappa \circ \sigma\left(C_{1}\right)$ and $\kappa \circ \sigma\left(C_{2}\right)$ are conics in $\mathbb{P}^{2}$, or $\kappa \circ \sigma\left(C_{1}\right)$ and $\kappa \circ \sigma\left(C_{2}\right)$ are lines in $\mathbb{P}^{2}$.

Suppose that $\kappa \circ \sigma\left(C_{1}\right)$ and $\kappa \circ \sigma\left(C_{2}\right)$ are lines in $\mathbb{P}^{2}$. Then the intersection $\kappa \circ \sigma\left(C_{1}\right) \cap \kappa \circ \sigma\left(C_{2}\right)$ consists of a single point in $R$, which implies that $\sigma\left(C_{1}\right) \cap \sigma\left(C_{2}\right)$ also consists of a single point that must be the unique singular point of the surface $\bar{X}$. So, we see that $\sigma\left(E_{1}\right)=\ldots=\sigma\left(E_{r}\right)$. If this point is not an ordinary double point of the surface $\bar{X}$, then $X \subset \mathbb{P}^{5}$ contains a $\bar{G}$-orbit of length at most 3, which is impossible, because $G$ is transitive. Hence, we see that $r=1$ and $\bar{X}$ has one singular point, which is an ordinary double point. In particular, the curve $E_{1}$ is $\bar{G}$-invariant. On the other hand, the curves $\sigma\left(C_{1}\right)$ and $\sigma\left(C_{2}\right)$ are curves in the linear system $\left|-K_{\bar{X}}\right|$ that are 
singular at the point $\sigma\left(E_{1}\right)$. Moreover, the multiplicity of the curves $\sigma\left(C_{1}\right)$ and $\sigma\left(C_{2}\right)$ at the point $\sigma\left(E_{1}\right)$ must equal 2 , which implies that $E_{1} \cdot C_{1}=E_{1} \cdot C_{2}=2$. Then

$$
H \cdot E_{1}=C_{1} \cdot E_{1}-K_{X} \cdot E_{1}=C_{2} \cdot E_{1}-K_{X} \cdot E_{1}=2,
$$

which is impossible, since $G$ is transitive. The obtained contradiction shows that the curves $\kappa \circ \sigma\left(C_{1}\right)$ and $\kappa \circ \sigma\left(C_{2}\right)$ are not lines in $\mathbb{P}^{2}$.

We see that $\kappa \circ \sigma\left(C_{1}\right)$ and $\kappa \circ \sigma\left(C_{2}\right)$ are conics in $\mathbb{P}^{2}$. Then the curves $\sigma\left(C_{1}\right)$ and $\sigma\left(C_{2}\right)$ are smooth, which implies that $C_{i} \cdot E_{j}=1$ for every $i$ and $j$. Then $H \cdot E_{i}=C_{j} \cdot E_{i}-K_{X} \cdot E_{i}=1$ for every $i$ and $j$, which implies that $r \geqslant 3$, since the group $G$ is transitive. If $\bar{X}$ has a $\bar{G}$-invariant singular point that is not an ordinary double point of the surface $\bar{X}$, then $X$ contains a $\bar{G}$-orbit of length at most 3 , which is impossible, because $G$ is transitive. On the other hand, we know that $\operatorname{rkPic}(\bar{X})+r=8$ by Theorem 4.3 , which implies that $3 \leqslant r \leqslant 7$. Since the singular points of the surface $\bar{X}$ are Du Val singular points, we see that either $\bar{X}$ has only ordinary double points, or the set $\operatorname{Sing}(\bar{X})$ consists of 2 singular points of type $\mathbb{A}_{3}$, or the set $\operatorname{Sing}(\bar{X})$ consists of 3 singular points of type $\mathbb{A}_{2}$. However, if the set $\operatorname{Sing}(\bar{X})$ consists of 2 singular points of type $\mathbb{A}_{3}$, then $X$ contains a $\bar{G}$-orbit of length at most 4 , which is impossible, because $G$ is transitive. Similarly, if the set $\operatorname{Sing}(\bar{X})$ consists of 3 singular points of type $\mathbb{A}_{2}$, then $X$ contains a $\bar{G}$-orbit of length at most 3 , which is impossible, because $G$ is transitive. Therefore, we see that $\bar{X}$ has only ordinary double points, which implies that the curves $E_{1}, \ldots, E_{r}$ are disjoint and all points $\kappa \circ \sigma\left(E_{1}\right), \ldots, \kappa \circ \sigma\left(E_{r}\right)$ are different. Note that $\kappa \circ \sigma\left(E_{1}\right), \ldots, \kappa \circ \sigma\left(E_{r}\right)$ are all singular points of the curve $R$, and they are ordinary double points of the curve $R$. Since

$$
\left\{\kappa \circ \sigma\left(E_{1}\right), \ldots, \kappa \circ \sigma\left(E_{r}\right)\right\} \subset \kappa \circ \sigma\left(C_{1}\right) \cap \kappa \circ \sigma\left(C_{2}\right),
$$

we see that $r \leqslant 4$, because the intersection $\kappa \circ \sigma\left(C_{1}\right) \cap \kappa \circ \sigma\left(C_{2}\right)$ consists of at most four points, and the curves $\kappa \circ \sigma\left(C_{1}\right)$ and $\kappa \circ \sigma\left(C_{2}\right)$ are conics in $\mathbb{P}^{2}$.

Suppose that there is a point $P \in \mathbb{P}^{2}$ that is fixed by $\beta(\bar{G})$. If $P \notin \operatorname{Sing}(R)$, then $X$ has $\bar{G}$-orbit of length at most 2 , which is impossible, because $G$ is transitive. If $P=\kappa \circ \sigma\left(E_{i}\right)$ for some $i \in\{1, \ldots, r\}$, then the intersection of the curve $E_{i}$ and a proper transform of the curve $\bar{R}$ on the surface $X$ is a $\bar{G}$-invariant set of two points, which is impossible, since $G$ is transitive. We see that there is no $\beta(\bar{G})$-invariant point in $\mathbb{P}^{2}$.

Suppose that $r=3$. Then $\bar{G}$ acts transitively on $|\operatorname{Sing}(R)|$, since there are no $\beta(\bar{G})$-fixed points in $\mathbb{P}^{2}$. One has $\sigma\left(C_{1}\right) \cdot \sigma\left(C_{1}\right)=\sigma\left(C_{2}\right) \cdot \sigma\left(C_{2}\right)=3 / 2$, which implies that the pencil $\left|K_{X}+H\right|$ is not $\xi$-invariant. In particular, we see that $\xi \notin \bar{G}$, which implies that $\bar{G} \cong \beta(\bar{G})$. Note that either $R$ is a union of a line and a nonsingular cubic curve, or $R$ is an irreducible plane quartic curve with three ordinary double points. In the former case each component of the curve $R$ must be $\beta(\bar{G})$-invariant, which implies that there exists a point in $\mathbb{P}^{2}$ that is fixed by $\beta(\bar{G})$, which is impossible. Thus, we see that $R$ is an irreducible plane quartic curve with three ordinary double points. In particular, the group $\beta(\bar{G})$ acts faithfully on the curve $R$. Let $v: \tilde{R} \rightarrow R$ be the normalization of the curve $R$. Then the faithful action of the group $\beta(\bar{G})$ on the curve $R$ induces a faithful action of the group $\beta(\bar{G})$ on the curve $\tilde{R}$. On the other hand, the curve $\tilde{R}$ is rational, which implies that either $\beta(\bar{G}) \cong \mathrm{S}_{4}$, or $\tilde{R}$ contains a $\beta(\bar{G})$-orbit of length at most 2. The former case is impossible by Lemma [5.1, since $\bar{G} \cong \beta(\bar{G})$. Hence, there is a $\beta(\bar{G})$-orbit $\Omega \subset \tilde{R}$ that consists of at most 2 points. Then $v(\Omega) \not \subset \operatorname{Sing}(R)$, since otherwise one of the points of $\operatorname{Sing}(R)$ would be fixed by $\beta(\bar{G})$, which is impossible. Hence, the surface $X$ contains a $\bar{G}$-orbit that consists of at most 2 points, which is impossible, since $G$ is transitive.

Thus, we see that $r=4$. Then either $R$ is a union of a line and an irreducible singular cubic curve, or $R$ is a union of two irreducible conics that intersect each other transversally. In the former case the exists a $\beta(\bar{G})$-fixed point in $\mathbb{P}^{2}$, which is impossible. Thus, we see that $R$ is union of two irreducible conics. Let us call them $T_{1}$ and $T_{2}$. Let $\tilde{T}_{1}$ and and $\tilde{T}_{2}$ be irreducible curves in $X$ such that $\kappa \circ \sigma\left(\tilde{T}_{1}\right)=T_{1}$ and $\kappa \circ \sigma\left(\tilde{T}_{2}\right)=T_{2}$. Then $\tilde{T}_{1} \cdot C_{1}=\tilde{T}_{1} \cdot C_{2}=\tilde{T}_{2} \cdot C_{1}=\tilde{T}_{2} \cdot C_{2}=0$, 
which implies that the curves $\tilde{T}_{1}$ and $\tilde{T}_{2}$ are contained in the pencil $\left|K_{X}+H\right|$. Note that in this case the pencil $\left|K_{X}+H\right|$ is $\langle\xi\rangle$-invariant, so we do not know whether $\xi$ is contained in $\bar{G}$ or not. On the other hand, we have $2 H \sim 2 \tilde{T}_{1}+2 \tilde{T}_{2}+\sum_{i=1}^{4} E_{i}$, and the divisor $2 \tilde{T}_{1}+2 \tilde{T}_{2}+\sum_{i=1}^{4} E_{i}$ is $\bar{G}$-invariant, which is impossible by Lemma 5.3 .

Lemma 5.13. If $X$ is a surface and $\operatorname{deg}(X)=7$, then $-K_{X} \cdot H \neq 3$.

Proof. Suppose that $X$ is a surface such that $\operatorname{deg}(X)=7$ and $-K_{X} \cdot H=3$. Then it follows from (5.8) and the Riemann-Roch theorem that $h^{0}\left(\mathcal{O}_{X}\left(K_{X}+H\right)\right) \geqslant 3$. One the other hand, there is an exact sequence of cohomology groups

$$
0 \rightarrow H^{0}\left(\mathcal{O}_{X}\left(K_{X}\right)\right) \rightarrow H^{0}\left(\mathcal{O}_{X}\left(K_{X}+H\right)\right) \rightarrow H^{0}\left(\mathcal{O}_{H}\left(K_{H}\right)\right) \cong \mathbb{C}^{3}
$$

which implies that $h^{0}\left(\mathcal{O}_{X}\left(K_{X}+H\right)\right) \leqslant 3$, since $h^{0}\left(\mathcal{O}_{X}\left(K_{X}\right)\right)=0$, because $X$ is of Fano type. Thus, we see that $h^{0}\left(\mathcal{O}_{X}\left(K_{X}+H\right)\right)=3$.

Suppose that there is a $\bar{G}$-invariant curve contained in the base locus of the linear system $\left|K_{X}+H\right|$. Since $\left(K_{X}+H\right) \cdot H=4$ and $\operatorname{dim}\left|K_{X}+H\right|>1$, this curve must be a disjoint union of three lines, because $G$ is transitive. Let us denote these lines by $L_{1}, L_{2}$, and $L_{3}$. Then $\bar{G}$ acts transitively on these lines. One has $K_{X}+H \sim L_{1}+L_{2}+L_{3}+L$, where $L$ is a line on $X$ such that $|L|$ is free from fixed components. In particular, the linear system $|L|$ has at most one base point since $L$ is a line, which implies that $|L|$ is base point free by the transitivity of $\bar{G}$, because the base locus of the linear system $|L|$ must be $\bar{G}$-invariant. Hence, we may assume that $L$ is contained in the smooth locus of the surface $X$, so that adjunction formula implies $-2=\left(K_{X}+L\right) \cdot L \geqslant-H \cdot L=-1$, which is a contradiction. Therefore, the base locus of the linear system $\left|K_{X}+H\right|$ consists of at most finitely many points.

Suppose that $\left|K_{X}+H\right|$ is not composed of a pencil. Let $C_{1}$ and $C_{2}$ be two general curves in $\left|K_{X}+H\right|$, let $\Lambda_{1}$ and $\Lambda_{2}$ be the linear spans of the curves $C_{1}$ and $C_{2}$ in $\mathbb{P}^{5}$. Then the curves $C_{1}$ and $C_{2}$ are irreducible by Bertini theorem, which implies that $\Lambda_{1}$ and $\Lambda_{2}$ are proper linear subspaces of $\mathbb{P}^{5}$. Note that $\Lambda_{1} \neq \Lambda_{2}$, since $G$ is transitive. Hence, the set $\Lambda_{1} \cap C_{2}$ consists of at most $\operatorname{deg}\left(C_{2}\right)=4$ points. Moreover, the base locus of the linear system $\left|K_{X}+H\right|$ is contained in $\Lambda_{1} \cap C_{2}$, which immediately implies that $\left|K_{X}+H\right|$ is base point free, since $G$ is transitive.

If $\left|K_{X}+H\right|$ is composed of some pencil $\mathcal{P}$, then arguing as in the case when $\left|K_{X}+H\right|$ is not composed of a pencil, we easily see the base locus of the pencil $\mathcal{P}$ consists of at most 4 points, which implies that $\left|K_{X}+H\right|$ is base point free, since the group $G$ is transitive and $\mathcal{P}$ must be $\bar{G}$-invariant. Thus, we can conclude that the linear system $\left|K_{X}+H\right|$ is base point free. In particular, the divisor $K_{X}$ is Cartier, which implies that $X$ has at most Du Val singularities, since we know already that $X$ has at most quotient singularities.

Note that $\left(7 K_{X}+3 H\right) \cdot H=0$. By Hodge index theorem one has $\left(7 K_{X}+3 H\right)^{2} \leqslant 0$, which implies that $K_{X}^{2} \leqslant 1$. Now we are going to show that $h^{0}\left(\mathcal{O}_{X}\left(-K_{X}\right)\right)=0$.

Suppose that $h^{0}\left(\mathcal{O}_{X}\left(-K_{X}\right)\right) \geqslant 2$. Since $G$ is transitive, $\left|-K_{X}\right|$ is $\bar{G}$-invariant and $-K_{X} \cdot H=3$, we see that the linear system $\left|-K_{X}\right|$ does not have fixed curves. In particular, the divisor $-K_{X}$ is nef. Recall that $-K_{X}$ is big, since $X$ is of Fano type. Therefore, the surface $X$ must be a weak del Pezzo surface. In particular, we have $K_{X}^{2} \geqslant 1$, which implies that $K_{X}^{2}=1$, because we already proved that $K_{X}^{2} \leqslant 1$. It is well known that $\left|-K_{X}\right|$ has a unique base point, which is impossible, since $G$ is transitive. Therefore, we see that $h^{0}\left(\mathcal{O}_{X}\left(-K_{X}\right)\right) \leqslant 1$.

Suppose that $h^{0}\left(\mathcal{O}_{X}\left(-K_{X}\right)\right)=1$. Since $-K_{X} \cdot H=3$ and $G$ is transitive, the only possibility is that the unique effective divisor $D$ in $\left|-K_{X}\right|$ must be a disjoint union of three lines, because $G$ is transitive. Since $-K_{X}$ is a Cartier divisor and $D$ is a smooth curve, we see that $D$ is contained in the smooth locus of the surface $X$. This immediately leads to a contradiction by adjunction formula. Therefore, we see that $h^{0}\left(\mathcal{O}_{X}\left(-K_{X}\right)\right) \neq 1$.

We see that $h^{0}\left(\mathcal{O}_{X}\left(-K_{X}\right)\right)=0$. Note that $h^{2}\left(\mathcal{O}_{X}\left(-K_{X}\right)\right)=h^{0}\left(\mathcal{O}_{X}\left(2 K_{X}\right)\right)$ by the Serre duality, and $h^{0}\left(\mathcal{O}_{X}\left(2 K_{X}\right)\right)=0$ since $H \cdot K_{X}<0$. Thus, the Riemann-Roch theorem implies 
that $h^{0}\left(\mathcal{O}_{X}\left(-K_{X}\right)\right) \geqslant 1+K_{X}^{2}$, so that $K_{X}^{2} \leqslant-1$. On the other hand, since the linear system $\left|K_{X}+H\right|$ is base point free, one has $0 \leqslant\left(K_{X}+H\right)^{2}=K_{X}^{2}+1$, which implies that $K_{X}^{2}=-1$ and $\left(K_{X}+H\right)^{2}=0$. Then the linear system $|K+H|$ gives us a morphism $\psi: X \rightarrow \mathbb{P}^{2}$ such that $\psi(X)$ is a curve. So the linear system $|K+H|$ is composed of a pencil. Note that $\psi(X)$ can not be a line. In fact, one can easily see that $\psi(X)$ must be a smooth conic, because $\left(K_{X}+H\right) \cdot H=4$. Indeed, the degree of the curve $\psi(X)$ is a number of irreducible components of a general element in $|K+H|$. Since $\left(K_{X}+H\right) \cdot H=4$, we see that $\psi(X)$ is either an irreducible conic or an irreducible quartic, since all irreducible components of a general element in $|K+H|$ must have the same degree in $\mathbb{P}^{5}$. If $\psi(X)$ is an irreducible quartic, then general curve in $\left|K_{X}+H\right|$ is a disjoint union of four lines in $\mathbb{P}^{5}$, which immediately leads to a contradiction with the adjunction formula. So, we see that $\psi(X)$ must be a smooth conic.

Let $C$ be a fiber of the morphism $\psi$ over a general point in $\psi(X)$. Then $C$ is an irreducible conic in $\mathbb{P}^{2}$, and $K_{X}+H \sim 2 C$. The pencil $|C|$ is base point free and it induces a morphism $\vartheta: X \rightarrow \mathbb{P}^{1}$, whose general fiber is an irreducible conic in $\mathbb{P}^{5}$. Thus, we obtain the diagram

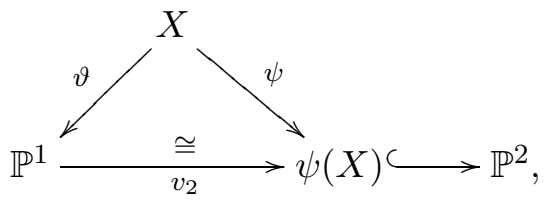

where $v_{2}: \mathbb{P}^{1} \rightarrow \mathbb{P}^{2}$ is the second Veronese embedding.

Let us prove that $X$ is smooth. Suppose that $X$ is singular. By Theorem 4.3 , we have

$$
\operatorname{rkPic}(X)+K_{X}^{2}+\sum_{P \in X} \mu(P)=10
$$

where $\mu(P)$ is the Milnor number of a point $P \in X$. Since $G$ is transitive, it follows from (5.14) that $|\operatorname{Sing}(X)| \geqslant 6$ and all singular points of the surface $X$ are ordinary double points. Let $r$ be the number of reducible fibers of the morphism $\vartheta$. Since $H \cdot C=2$, every reducible fiber of the morphism $\vartheta$ consists of two different lines in $\mathbb{P}^{5}$ intersecting transversally in one point. In particular, there is a $\bar{G}$-invariant subset in $X$ that consists of exactly $r$ points, which implies that either $r=0$ or $r \geqslant 6$. Note that $\operatorname{rkPic}(X)=r+2$. Therefore $r=0$, since otherwise it would follow from (5.14) that $10=r+|\operatorname{Sing}(X)|+1 \geqslant 13$, which is contradiction. Since $r=0$, it follows from (5.14) that $|\operatorname{Sing}(X)|=9$. Since every fiber of the morphism $\vartheta$ is irreducible rational curve and $X$ has only ordinary double points, every fiber of $\vartheta$ contains either none or two singular points of the surface $X$. Thus, nine singular points of the surface $X$ must split in pairs, which is impossible. Therefore, the surface $X$ is smooth.

Note that $h^{2}\left(\mathcal{O}_{X}\left(C-2 K_{X}\right)\right)=h^{0}\left(\mathcal{O}_{X}\left(3 K_{X}-C\right)\right)$ by the Serre duality. Thus the RiemannRoch theorem implies that

$$
h^{0}\left(\mathcal{O}_{X}\left(C-2 K_{X}\right)\right) \geqslant 1+\frac{\left(C-2 K_{X}\right) \cdot\left(C-3 K_{X}\right)}{2}=3
$$

because $h^{0}\left(\mathcal{O}_{X}\left(3 K_{X}-C\right)\right)=0$, since $H \cdot\left(3 K_{X}-C\right)=-11<0$. Now we are going to prove that the linear system $\left|C-2 K_{X}\right|$ has no base curves.

Suppose that the linear system $\left|C-2 K_{X}\right|$ contains a fixed curve. Then there exists a nonzero effective divisor $Z$ on the surface $X$ such that the linear system $\left|C-2 K_{X}-Z\right|$ has no base curves, i. e. $Z$ is a union of one dimensional components of the base locus of the linear system $\left|C-2 K_{X}\right|$ taken with with appropriate multiplicities. Then $Z$ must be $\bar{G}$-invariant, which implies that $H \cdot Z \geqslant 3$, since the group $G$ is transitive. On the other hand, we have $H \cdot Z<H \cdot\left(C-2 K_{X}\right)=8$, since $h^{0}\left(\mathcal{O}_{X}\left(C-2 K_{X}\right)\right)>1$. Let $M$ be a general curve in the linear system $\left|C-2 K_{X}-Z\right|$. Then $1 \leqslant M \cdot H \leqslant 5$. Note that $K_{X} \cdot M \leqslant-1$, because $|M|$ is 
free from base curves and $-K_{X}$ is big, since $X$ is of Fano type. Thus, we have

$$
0 \leqslant 2 C \cdot M=\left(K_{X}+H\right) \cdot M \leqslant H \cdot M-1 .
$$

Suppose that $H \cdot M \leqslant 2$. Then $C \cdot M=0$ by (5.15), which implies that $M$ is contained in the fibers of the morphism $\vartheta$. If $H \cdot M=1$, this means that $M$ is a component of a reducible fiber of the morphism $\vartheta$, which is impossible since $M^{2} \geqslant 0$. If $H \cdot M=2$, then $M$ cannot consist of two components of different reducible fibers of the morphism $\vartheta$, because $M^{2} \geqslant 0$. Thus, if $H \cdot M=2$, then $M \in|C|$, since $H \cdot M=H \cdot C$, which is impossible, since

$$
h^{0}\left(\mathcal{O}_{X}\left(C-2 K_{X}\right)\right) \geqslant 3>2=h^{0}\left(\mathcal{O}_{X}(C)\right) .
$$

Therefore, we see that $H \cdot M \geqslant 3$.

Suppose that $H \cdot M=3$. Arguing as above, we see that $M$ is not contained in the fibers of the morphism $\vartheta$. Thus, we must have $C \cdot M \geqslant 1$, so that $C \cdot M=1$ by (5.15). In particular, the linear system $|M|$ is not composed of a pencil unless $|M|$ is a pencil itself, since otherwise one would have $C \cdot M \geqslant 2$. In particular, the curve $M$ is irreducible by the Bertini theorem. Therefore, the curve $M$ is a section of the morphism $\vartheta$, which implies that $M$ is smooth and rational. Applying the adjunction formula to the curve $M$, one obtains

$$
-2=\left(K_{X}+M\right) \cdot M=-1+M^{2} \geqslant-1,
$$

which is a contradiction. Thus, we see that $H \cdot M \neq 3$.

Suppose that $H \cdot M=4$ and $C \cdot M \geqslant 1$. Then $C \cdot M=1$ by (5.15). In particular, the linear system $|M|$ is not composed of a pencil unless $|M|$ is a pencil itself, since otherwise one would have $C \cdot M \geqslant 2$. Hence, the curve $M$ is irreducible by the Bertini theorem. Therefore, the curve $M$ is a section of the morphism $\vartheta$, which implies that $M$ is smooth and rational. Applying the adjunction formula to the curve $M$, wee see that $-2=\left(K_{X}+M\right) \cdot M=-2+M^{2}$, which implies that $M^{2}=0$. Hence, the linear system $|M|$ is composed of a pencil, which implies that $|M|$ is a pencil, since $M$ is irreducible. The latter is impossible since we already proved that $h^{0}\left(\mathcal{O}_{X}(M)\right) \geqslant 3$. Thus, we see that if $H \cdot M=4$, then $C \cdot M=0$.

Suppose that $H \cdot M=4$ and $C \cdot M=0$. Since $|M|$ is free from base curves, we must have $M \sim 2 C$, because $H \cdot M=2 H \cdot C$. Note that $H \cdot Z=4$. Thus, since the group $G$ is transitive, the divisor $Z$ is either a union of two different conics in $\mathbb{P}^{5}$ or a union of four different lines in $\mathbb{P}^{5}$. Let us consider these cases separately.

Suppose that $Z$ is a union of two different conics in $\mathbb{P}^{5}$. Let us denote these conics by $R_{1}$ and $R_{2}$. Let $\Lambda_{1}$ and $\Lambda_{2}$ be linear spans in $\mathbb{P}^{5}$ of the conics $R_{1}$ and $R_{2}$. Then $\Lambda_{1} \cap \Lambda_{2}=\varnothing$, because $G$ is transitive and $Z$ is $\bar{G}$-invariant. On the other hand, we have $C \cdot Z=4$, which implies that both intersections $C \cap R_{1}$ and $C \cap R_{2}$ consist of two points, because the conic $C$ is a fiber of the morphism $\psi$ over a general point in $\psi(X)$. Thus, the linear span in $\mathbb{P}^{5}$ of the conic $C$ intersects both linear subspaces $\Lambda_{1}$ and $\Lambda_{2}$ by lines, which is impossible since $\Lambda_{1}$ and $\Lambda_{2}$ are disjoint. Thus, we see that $Z$ can not be a union of two different conics in $\mathbb{P}^{5}$.

We see that $Z$ is a union of four different lines. Let us denote these lines by $L_{1}, L_{2}, L_{3}$, and $L_{4}$. Then $\bar{G}$ acts transitively on these lines, because $G$ is transitive. It follows from the Riemann-Roch theorem that

$$
h^{0}\left(\mathcal{O}_{X}(H-C)\right) \geqslant 1+\frac{(H-C) \cdot\left(H-C-K_{X}\right)}{2}=3
$$

since $h^{2}\left(\mathcal{O}_{X}(H-C)\right)=h^{0}\left(\mathcal{O}_{X}\left(K_{X}-H+C\right)\right)$ by the Serre duality, and $h^{0}\left(\mathcal{O}_{X}\left(K_{X}-H+C\right)\right)=0$, because $H \cdot\left(K_{X}-H+C\right)=-8<0$. By Theorem 4.3, we have $\rho(X)=11$, which implies that the morphism $\vartheta$ has 9 reducible fibers. Thus, it follows from Lemma 4.1 that there exist two curves $C_{1}$ and $C_{2}$ in the pencil $|C|$ such that the divisor $C_{1}+C_{2}$ is $\bar{G}$-invariant. Since $G$ is transitive, we see that $C_{1} \cup C_{2}$ is not contained in a hyperplane in $\mathbb{P}^{5}$. In particular, one has 
$h^{0}\left(\mathcal{O}_{X}(H-2 C)\right)=0$. On the other hand, there is an exact sequence of cohomology groups

$$
0 \rightarrow H^{0}\left(\mathcal{O}_{X}(H-2 C)\right) \rightarrow H^{0}\left(\mathcal{O}_{X}(H-C)\right) \rightarrow H^{0}\left(\mathcal{O}_{C}(H-C)\right)
$$

which implies that $h^{0}\left(\mathcal{O}_{X}(H-C)\right)=3$, since $h^{0}\left(\mathcal{O}_{X}(H-2 C)\right)=0, h^{0}\left(\mathcal{O}_{C}(H-C)\right)=3$ and $h^{0}\left(\mathcal{O}_{X}(H-C)\right) \geqslant 3$. On the other hand, one can easily see that the linear system $|H-C|$ is base point free, since the linear spans in $\mathbb{P}^{5}$ of the conics $C_{1}$ and $C_{2}$ are disjoint. Furthermore, the linear system $|H-C|$ is not composed of a pencil since $(H-C)^{2}=3>0$. Let $\tau: X \rightarrow \mathbb{P}^{2}$ be a morphism that is given by the linear system $|H-C|$. Then $\tau$ is $\bar{G}$-equivariant, surjective and generically three-to-one. Note that $\tau$ can be considered as a projection from a two dimensional linear subspace in $\mathbb{P}^{5}$. Since $L_{i} \cdot(H-C)=0$ for every $i \in\{1,2,3,4\}$, the lines $L_{1}, L_{2}, L_{3}$, and $L_{4}$ are contracted by $\tau$. Moreover, the points $\tau\left(L_{1}\right), \tau\left(L_{2}\right), \tau\left(L_{3}\right)$, and $\tau\left(L_{4}\right)$ are different and are not contained in a line in $\mathbb{P}^{2}$, because $L_{1} \cup L_{2} \cup L_{3} \cup L_{4}$ is not contained in a hyperplane in $\mathbb{P}^{5}$, since $G$ is transitive. Let $\iota: \bar{G} \rightarrow \operatorname{Aut}\left(\mathbb{P}^{2}\right)$ be the homomorphism induced by the morphism $\tau$. Then $\iota(\bar{G})$ acts faithfully on the set $\left\{\tau\left(L_{1}\right), \tau\left(L_{2}\right), \tau\left(L_{3}\right), \tau\left(L_{4}\right)\right\}$ since every element in Aut $\left(\mathbb{P}^{2}\right)$ is defined by the images of 4 points in general position. In particular, we see that $\iota(\bar{G})$ is a subgroup of the group $\mathrm{S}_{4}$, which implies that there is a $\iota(\bar{G})$-invariant conic in $\mathbb{P}^{2}$. Therefore, there exists a $\bar{G}$-invariant divisor in the linear system $|2 H-2 C|$. Let us denote this divisor by $B$. Then $B+C_{1}+C_{2} \sim 2 H$, and $B+C_{1}+C_{2}$ is $\bar{G}$-invariant, which is impossible by Lemma 5.3 . The obtained contradiction shows that $H \cdot M \neq 4$.

Suppose that $H \cdot M=5$. Then $H \cdot Z=3$, which implies that the curve $Z$ is a union of three lines transitively interchanged by $\bar{G}$, because $G$ is transitive. Let us denote these lines by $L_{1}, L_{2}$ and $L_{3}$. We have $3 L_{j} \cdot C=4-C \cdot M$ for every $j \in\{1,2,3\}$. Since $0 \leqslant C \cdot M \leqslant 4$ by (5.15), we see that $C \cdot M=1$. In particular, the linear system $|M|$ is not composed of a pencil unless $|M|$ is a pencil itself, since otherwise one would have $C \cdot M \geqslant 2$. Hence, the curve $M$ is irreducible by the Bertini theorem. Therefore, the curve $M$ is a section of the morphism $\vartheta$, which implies that $M$ is smooth and rational. Furthermore, we have $K_{X} \cdot M=(2 C-H) \cdot M=-3$. Now applying the adjunction formula to $M$, we see that $-2=\left(K_{X}+M\right) \cdot M=-3+M^{2}$, which implies that $M^{2}=1$. Thus, it follows from the Riemann-Roch theorem that

$$
h^{0}\left(\mathcal{O}_{X}(M-C)\right) \geqslant 1+\frac{(M-C) \cdot\left(M-C-K_{X}\right)}{2}=1
$$

because $h^{2}\left(\mathcal{O}_{X}(M-C)\right)=h^{0}\left(\mathcal{O}_{X}\left(K_{X}-M+C\right)\right)=0$ by the Serre duality, since one has $H \cdot\left(K_{X}-M+C\right)=-6<0$. Keeping in mind that $(M-C)^{2}=-1$, we see that the base locus of the linear system $|M-C|$ contains a $\bar{G}$-invariant curve. Let us denote this curve by $R$. Then $H \cdot R \leqslant H \cdot(M-C)=3$, which implies that the curve $R$ is a union of three lines transitively interchanged by $\bar{G}$, since $G$ is transitive. Let us denote these lines by $L_{1}^{\prime}, L_{2}^{\prime}$ and $L_{3}^{\prime}$. Then

$$
1=C \cdot M=C \cdot(M-C)=C \cdot\left(L_{1}^{\prime}+L_{2}^{\prime}+L_{3}^{\prime}\right)=3 C \cdot L_{1}^{\prime}=3 C \cdot L_{2}^{\prime}=3 C \cdot L_{2}^{\prime},
$$

which is a contradiction. Therefore $H \cdot M \neq 5$.

Thus, the assumption that $\left|C-2 K_{X}\right|$ has a fixed curve implies that $H \cdot M>5$. However, we proved earlier that $1 \leqslant M \cdot H \leqslant 5$. So, we see that $\left|C-2 K_{X}\right|$ has no based components, i.e. the curve $Z$ does not exists. On the other hand, one has $-K_{X} \cdot\left(C-2 K_{X}\right)=0$, which is impossible, because the divisor $-K_{X}$ is big, since the surface $X$ is of Fano type. The obtained contradiction completes the proof of Lemma 5.13 .

Now using Lemmas 5.9, 5.10, 5.11, and 5.13, we are ready to prove the following

Lemma 5.16. The subvariety $X$ is not a surface.

Proof. Suppose that $X$ is a surface. Then it follows from the Riemann-Roch theorem that

$$
h^{0}\left(\mathcal{O}_{X}(n H)\right)=\chi\left(\mathcal{O}_{X}(n H)\right)=1+\frac{n^{2}}{2}(H \cdot H)-\frac{n}{2}\left(H \cdot K_{X}\right)
$$


for any $n \geqslant 1$. In particular, since $X$ is not contained in a hyperplane in $\mathbb{P}^{5}$, it follows from (5.7) that $H \cdot H-H \cdot K_{X}=10$. On the other hand, we know that $H \cdot H \geqslant 4$, since $S$ is not contained in a hyperplane in $\mathbb{P}^{5}$. Moreover, since $X$ is of Fano type, the divisor $-K_{X}$ is big, which implies that $-H \cdot K_{X} \geqslant 1$. Thus, we see that $H \cdot H \in\{4,5,6,7,8,9\}$. Now plugging $n=2$ into (5.7) and (5.17), we see that $h^{0}\left(\mathcal{O}_{\mathbb{P}^{5}}(2) \otimes \mathcal{I}_{X}\right)=10-H \cdot H$. Then $H \cdot H \leqslant 7$ by Lemma 5.6, which implies that $H \cdot H \in\{4,5,6,7\}$. Since $H \cdot H-H \cdot K_{X}=10$, we immediately obtain a contradiction using Lemmas 5.9, 5.10, 5.11, and 5.13,

Thus, we see that $X$ is a threefold. Put $d=H \cdot H \cdot H$.

Lemma 5.18. The inequality $d \geqslant 5$ holds.

Proof. Since $G$ is transitive, the threefold $X$ is not contained in a hyperplane. Furthermore, the threefold $X$ is not a cone since the vertex of a cone is a linear space, which again contradicts transitivity of $\bar{G}$. Hence it follows from [54, Theorem 1] that $X$ is either a complete intersection of two quadrics, or a projection from $\mathbb{P}^{6}$ of a hyperplane section of the Segre variety $\mathbb{P}^{1} \times \mathbb{P}^{3} \subset \mathbb{P}^{7}$. The former case is impossible by assumption, and the latter case is impossible since $X$ is projectively normal.

By Lemma 5.18 the threefold $X$ is not contained in an intersection of two quadrics in $\mathbb{P}^{5}$ (indeed, otherwise either $X$ would be contained in a hyperplane, or one would have $d \leqslant 4$ ). On the other hand, $h^{0}\left(\mathcal{O}_{\mathbb{P}^{5}}(2) \otimes \mathcal{I}_{X}\right) \neq 1$ since $\bar{G}$ does not have invariant quadrics in $\mathbb{P}^{5}$. Therefore, we have the following

Corollary 5.19. The equality $h^{0}\left(\mathcal{O}_{\mathbb{P}^{5}}(2) \otimes \mathcal{I}_{X}\right)=0$ holds.

Recall that $f: \tilde{X} \rightarrow X$ is a resolution of singularities of the threefold $X$. Let $\mathrm{c}_{2}(\tilde{X})$ be the second Chern class of the threefold $\tilde{X}$. Put $\gamma=f^{*}(H) \cdot\left(K_{\tilde{X}} \cdot K_{\tilde{X}}+\mathrm{c}_{2}(\tilde{X})\right)$ and $k=-H \cdot H \cdot K_{X}$. Then $k \geqslant 1$, since $X$ is of Fano type. Then

$$
h^{0}\left(\mathcal{O}_{X}(n H)\right)=\chi\left(\mathcal{O}_{X}(n H)\right)=d \frac{n^{3}}{6}+k \frac{n^{2}}{4}+\frac{n}{12} \gamma+1
$$

by the Riemann-Roch theorem. Using (5.7) and Corollary 5.19, we see that

$$
\left\{\begin{array}{l}
\frac{d}{6}+\frac{k}{4}+\frac{\gamma}{12}+1=6 \\
\frac{4 d}{3}+k+\frac{\gamma}{6}+1=21 \\
\frac{9 d}{2}+\frac{9 k}{4}+\frac{\gamma}{4}+1=56-h^{0}\left(\mathcal{O}_{\mathbb{P}^{5}}(3) \otimes \mathcal{I}_{X}\right),
\end{array}\right.
$$

which implies that $10=d+k / 2$ and $h^{0}\left(\mathcal{O}_{\mathbb{P}^{5}}(3) \otimes \mathcal{I}_{X}\right)=k / 2$. Keeping in mind that $d \geqslant 5$ by Lemma 5.18 and $k \geqslant 1$, we see that $d \in\{5,6,7,8,9\}$.

Note that $d \neq 9$, since otherwise one would have $h^{0}\left(\mathcal{O}_{\mathbb{P}^{5}}(3) \otimes \mathcal{I}_{X}\right)=1$ so that $\bar{G}$ would have an invariant cubic in $\mathbb{P}^{5}$ which is not the case by assumption. Finally, if $d=8$, then $h^{0}\left(\mathcal{O}_{\mathbb{P}^{5}}(3) \otimes \mathcal{I}_{X}\right)=2$ so that there is a $\bar{G}$-invariant pencil $\mathcal{P}$ of cubics in $\mathbb{P}^{5}$. For two different hypersurfaces $R_{1}, R_{2} \in \mathcal{P}$ the intersection $R_{1} \cap R_{2}$ consists of the threefold $X$ and a threefold $T$ of degree $\operatorname{deg}(T)=9-d=1$. Thus $T$ is a $\bar{G}$-invariant projective subspace of $\mathbb{P}^{5}$, which is impossible by assumption.

The next two lemmas deal with the (slightly more difficult) cases $d=5$ and $d=7$.

Lemma 5.21. The inequality $d \neq 5$ holds.

Proof. Suppose that $d=5$. Then $k=10$, because $10=d+k / 2$. Since $\left(K_{X}+2 H\right) \cdot H^{2}=0$, the sectional genus of $X$ is 1 . Note that $X$ is rationally connected by [58], because $X$ is of Fano 
type. Applying [22, Theorem 5.4] and keeping in mind that $X$ can be neither a scroll over an elliptic curve nor a generalized cone over such scroll, we get

$$
1=3+\operatorname{deg}(X)-h^{0}\left(\mathcal{O}_{X}(H)\right)=2,
$$

which is a contradiction.

Lemma 5.22. The inequality $d \neq 7$ holds.

Proof. Suppose that $d=7$. Then $k=6$ and $h^{0}\left(\mathcal{O}_{\mathbb{P}^{5}}(3) \otimes \mathcal{I}_{X}\right)=3$, because $10=d+k / 2$ and $h^{0}\left(\mathcal{O}_{\mathbb{P}^{5}}(3) \otimes \mathcal{I}_{X}\right)=k / 2$. Thus, an intersection of $X$ with a general linear subspace in $\mathbb{P}^{5}$ of codimension 2 is a smooth curve of genus 5 and degree 7 (cf. Theorem A.1).

Let $\mathcal{P}$ be the linear system of cubic hypersurfaces in $\mathbb{P}^{5}$ that contains $X$. Then $X$ is the only threefold in the base locus of the linear system $\mathcal{P}$, because $G$ is transitive. Let $Y_{1}, Y_{2}, Y_{3}$ be three general hypersurfaces in $\mathcal{P}$. Then $Y_{1} \cap Y_{2}=X \cup Q$, where $Q$ is a threefold of degree $\operatorname{deg}(Q)=2$. Moreover, it follows from Corollary $\mathrm{A.3}$ that $Q$ is a (reduced) irreducible quadric threefold.

Let $H\left(Y_{1}, Y_{2}\right)$ be the unique hyperplane in $\mathbb{P}^{5}$ that contains $Q$. Then

$$
\bigcap_{Y_{1}, Y_{2}} H\left(Y_{1}, Y_{2}\right)=\varnothing
$$

since otherwise this intersection would be a $\bar{G}$-invariant proper linear subspace of $\mathbb{P}^{5}$, which is impossible, because $G$ is transitive.

Put $S=Q \cap Y_{3}$. Then $S$ is a reduced surface of degree 6 by Corollary A.3. On the other hand, the intersection $H\left(Y_{1}, Y_{2}\right) \cap X$ is a surface that contains the surface $S$. Therefore, the scheme-theoretic intersection $H\left(Y_{1}, Y_{2}\right) \cdot X$ is reduced and consists of a union of a surface $S$ and some two-dimensional linear subspace $\Pi \subset \mathbb{P}^{5}$. (Note that the existence of the plane $\Pi$ follows form [42, Proposition 8] in the case when $X$ is smooth.)

A priori, the plane $\Pi$ depends on the choice of the divisors $Y_{1}, Y_{2}$, and $Y_{3}$ in the linear system $\mathcal{P}$. In fact, the plane $\Pi$ must vary when one varies the divisors $Y_{1}, Y_{2}$, and $Y_{3}$ in the linear system $\mathcal{P}$, because $G$ is transitive. Hence, the surface $H$ is swept out by lines, which implies that its the Kodaira dimension $\kappa(H)$ is $-\infty$.

Let $R$ be the general hyperplane section of $H \subset X \subset \mathbb{P}^{5}$. Then $R \cdot K_{H}=d-k$, which implies that $H \cdot K_{H}=1$. Thus, we have $\chi\left(\mathcal{O}_{H}(R)\right)=\chi\left(\mathcal{O}_{H}\right)+3$ by the Riemann-Roch theorem. Since $H$ is projectively normal, we have $h^{0}\left(\mathcal{O}_{H}(R)\right)=5$. On the other hand, we know that $h^{1}\left(\mathcal{O}_{H}(R)\right)=h^{2}\left(\mathcal{O}_{H}(R)\right)=0$. Since $\chi\left(\mathcal{O}_{H}(R)\right)=\chi\left(\mathcal{O}_{H}\right)+3$, one has $\chi\left(\mathcal{O}_{H}\right)=2$, which implies that $h^{2}\left(\mathcal{O}_{H}\right) \geqslant 1$. Let $\iota: \bar{H} \rightarrow H$ be a resolution of singularities. Then $h^{2}\left(\mathcal{O}_{\bar{H}}\right)=h^{2}\left(\mathcal{O}_{H}\right)$, because $H$ has rational singularitis. But $h^{0}\left(\mathcal{O}_{\bar{H}}\left(K_{X}\right)\right)=h^{2}\left(\mathcal{O}_{\bar{H}}\right)$ by the Serre duality, which implies that $\bar{H}$ is a surface of general type, which is impossible since $\kappa(H)=\kappa(\bar{H})=-\infty$.

Therefore, we see that $d=6$. Then $k=8$ and $h^{0}\left(\mathcal{O}_{\mathbb{P}^{5}}(3) \otimes \mathcal{I}_{X}\right)=4$, because $10=d+k / 2$ and $h^{0}\left(\mathcal{O}_{\mathbb{P}^{5}}(3) \otimes \mathcal{I}_{X}\right)=k / 2$, which implies that the sectional genus of the threefold $X$ is 3 by the adjunction formula. Thus, we proved that $X$ is a irreducible $\bar{G}$-invariant projectively normal nondegenerate Fano type threefold of degree 6 and sectional genus 3 such that $h^{0}\left(\mathcal{O}_{\mathbb{P}^{5}}(2) \otimes \mathcal{I}_{X}\right)=0$ and $h^{0}\left(\mathcal{O}_{\mathbb{P}^{5}}(3) \otimes \mathcal{I}_{X}\right)=4$. This completes the proof of Theorem 5.2 .

\section{Appendix A. Curves of genus 5 And Degree 7}

Let $C$ be a smooth irreducible curve in $\mathbb{P}^{3}$ of genus 5 and degree 7 , let $\pi: X \rightarrow \mathbb{P}^{3}$ be a blow up of the curve $C$, let $E$ be the $\pi$-exceptional divisor, and let $H$ be a general hyperplane in $\mathbb{P}^{3}$.

Theorem A.1. The divisor $-K_{X}$ is ample, and $\left|\pi^{*}(3 H)-E\right|$ is free from base points and induces a morphism $\phi: X \rightarrow \mathbb{P}^{2}$ that is a conic bundle with a discriminant curve of degree 5 . 
Proof. The required assertion is well-known in the case when $C$ is a scheme-theoretic intersection of cubic hypersurfaces in $\mathbb{P}^{3}$ (see [39, Proposition 7.5]). It is known that generic smooth connected curve in $\mathbb{P}^{3}$ of genus 5 and degree 7 is a scheme-theoretic intersection of cubic hypersurfaces in $\mathbb{P}^{3}$ (see [39, Corollary 6.2], [39, Proposition 7.5]). Unfortunately, we failed to find any reference with a proof that the same holds for any smooth connected curve in $\mathbb{P}^{3}$ of genus 5 and degree 7 . So we decided to prove it here.

Recall that the curve $C$ is called $m$-regular in the case when

$$
H^{1}\left(\mathcal{O}_{\mathbb{P}^{3}}(m-1) \otimes \mathcal{I}_{C}\right)=H^{2}\left(\mathcal{O}_{\mathbb{P}^{3}}(m-2) \otimes \mathcal{I}_{C}\right)=0,
$$

where $\mathcal{I}_{C}$ is the ideal sheaf of $C$. It is well-known that $C$ is a scheme-theoretic intersection of hypersurfaces of degree $m$ in $\mathbb{P}^{3}$ if it is $m$-regular (see [19, Excersize 20.21]). However, our curve $C$ is not 3 -regular, since $h^{2}\left(\mathcal{O}_{\mathbb{P}^{3}}(1) \otimes \mathcal{I}_{C}\right)=1$. On the other hand, it follows from [3, Exercise D.14(6)] that the curve $C$ is projectively normal, which implies that $h^{1}\left(\mathcal{O}_{\mathbb{P}^{3}}(n) \otimes \mathcal{I}_{C}\right)=0$ for every non-negative integer $n$. Moreover, it follows from the Riemann-Roch theorem that $h^{2}\left(\mathcal{O}_{\mathbb{P}^{3}}(2) \otimes \mathcal{I}_{C}\right)=0$, which implies that $C$ is 4 -regular. Thus, the curve $C$ is a schemetheoretic intersection of quartic hypersurfaces in $\mathbb{P}^{3}$. In particular, the divisor $-K_{X}$ is nef. Since $-K_{X}^{3}=16$ (this is obvious), we see that $-K_{X}$ is big and nef.

It follows from the projective normality of the curve $C$ that $C$ is not contained in any quadric hypersurface in $\mathbb{P}^{3}$ and $h^{0}\left(\mathcal{O}_{\mathbb{P}^{3}}(3) \otimes \mathcal{I}_{C}\right)=3$, which implies that $h^{0}\left(\mathcal{O}_{X}\left(\pi^{*}(3 H)-E\right)\right)=3$.

Let $P$ be a sufficiently general point in $\mathbb{P}^{3}$ that is not contained in the image under the morphism $\pi$ of the base locus of the linear system $\left|\pi^{*}(3 H)-E\right|$, and let $S_{1}$ and $S_{2}$ be two general cubic surfaces in $\mathbb{P}^{3}$ that pass through $C$ and contain $P$. Then $S_{1} \cdot S_{2}=C+\Delta$, where $\Delta$ is an effective one-cycle in $\mathbb{P}^{3}$ such that $P \in \operatorname{Supp}(\Delta)$. Since $H \cdot \Delta=2$, we see that $C \not \subset \operatorname{Supp}(\Delta)$, which implies that $S_{1}$ and $S_{2}$ are smooth in the general point of the curve $C$, the base locus of the linear system $\left|\pi^{*}(3 H)-E\right|$ does not contain fixed components and does not contain curves in $E$ that are not contracted by $\pi$ to points in $C$.

To complete the proof it is enough to prove that $\left|\pi^{*}(3 H)-E\right|$ is base point free. Note that the linear system $\left|\pi^{*}(3 H)-E\right|$ is base point free if its base locus does not contains curves that are not contained in $E$, since $\left(\pi^{*}(3 H)-E\right)^{3}=0$ and $\left|\pi^{*}(3 H)-E\right|$ does not contain curves in $E$ that are not contracted by $\pi$ to points in $C$. In particular, we see that $\left|\pi^{*}(3 H)-E\right|$ is base point free if $C$ is a set-theoretic intersection of cubic hypersurfaces in $\mathbb{P}^{3}$.

Suppose that the base locus of the linear system $\left|\pi^{*}(3 H)-E\right|$ contains an irreducible curve $\tilde{Z}$ such that $\tilde{Z} \not \subset E$. Let us show that this assumption leads to a contradiction.

Let $\tilde{S}_{1}$ and $\tilde{S}_{2}$ be the proper transforms of the surfaces $S_{1}$ and $S_{2}$ on the threefold $X$, respectively. Then $\tilde{S}_{1}$ and $\tilde{S}_{2}$ are general surfaces in $\left|\pi^{*}(3 H)-E\right|$. Let $\tilde{\Delta}$ be the proper transform of the one-cycle $\Delta$ on the threefold $X$. Then $\tilde{S}_{1} \cdot \tilde{S}_{2}=\tilde{\Delta}+\tilde{F}$, where $\tilde{F}$ is an effective one-cycle on $X$ whose support consists of finitely many curves in $X$ that are contracted by $\pi$ to points in $C$. By assumption, we know that $\tilde{Z} \subset \tilde{\Delta}$. Put $Z=\pi(\tilde{Z})$. Then $Z \neq \Delta$, since $P \notin Z$ and $P \in \operatorname{Supp}(\Delta)$. We see that $Z$ is a line and $\tilde{Z}$ the unique curve in the base locus of the linear system $\left|\pi^{*}(3 H)-E\right|$ that is not contained in the surface $E$, because $2=H \cdot \Delta>H \cdot Z \geqslant 1$. In particular, we see that $\Delta$ is reduced.

Let us show that the divisor $\pi^{*}(3 H)-E$ is nef. Suppose that this is not the case. Then $\left(\pi^{*}(3 H)-E\right) \cdot \tilde{Z}<0$. But $0>\left(\pi^{*}(3 H)-E\right) \cdot \tilde{Z}=3-E \cdot \tilde{Z}$ and $4-E \cdot \tilde{Z}=-K_{X} \cdot \tilde{Z} \geqslant 0$, which implies that $Z$ is a 4 -section of the curve $C$. Since it follows from the Riemann-Roch theorem that $h^{0}\left(\mathcal{O}_{C}\left(K_{C}-\left.H\right|_{C}\right)\right)=1$, the existence of a 4 -section to the curve $C$ immediately implies that the curve $C$ is either trigonal or hyperelliptic, which is not the case (see the proof of [39, Corollary 6.2]).

Thus, we see that $\pi^{*}(3 H)-E$ is nef. In particular, the divisor $-K_{X}$ is ample. Then $\tilde{F}=0$, because $\left(\pi^{*}(3 H)-E\right)^{3}=0$ and $\pi^{*}(3 H)-E$ is $\pi$-ample. Similarly, we see the base locus of the linear system $\left|\pi^{*}(3 H)-E\right|$ consists of the curve $\tilde{Z}$. We have $\tilde{\Delta}=\tilde{Z}+\tilde{R}$, where $\tilde{R}$ is an 
irreducible curve on $X$ such that $\pi(\tilde{R})$ is a line. Then

$$
0=\left(\pi^{*}(3 H)-E\right)^{3}=\left(\pi^{*}(3 H)-E\right) \cdot \tilde{S}_{1} \cdot \tilde{S}_{2}=\left(\pi^{*}(3 H)-E\right) \cdot \tilde{Z}+\left(\pi^{*}(3 H)-E\right) \cdot \tilde{R},
$$

which implies that $\left(\pi^{*}(3 H)-E\right) \cdot \tilde{Z}=\left(\pi^{*}(3 H)-E\right) \cdot \tilde{R}=0$, because $\pi^{*}(3 H)-E$ is nef. In particular, the curves $\tilde{R}$ and $\tilde{Z}$ both generate the extremal ray of the cone of effective cycles $\overline{\mathrm{NE}}(X)$ that is different from the ray contracted by $\pi$. Put $R=\pi(\tilde{R})$. Then both lines $Z$ and $R$ must be 3-secants of the curve $C$, because $\left(\pi^{*}(3 H)-E\right) \cdot \tilde{Z}=\left(\pi^{*}(3 H)-E\right) \cdot \tilde{R}=0$.

Since the base locus of the linear system $\left|\pi^{*}(3 H)-E\right|$ consists of the curve $\tilde{Z}$, we must have $\tilde{R} \cap \tilde{Z}=\varnothing$, because $\left(\pi^{*}(3 H)-E\right) \cdot \tilde{R}=0$. Then $R \cap Z=\varnothing$. Indeed, suppose that $R \cap Z \neq \varnothing$. Then $R \cap Z$ is a point contained in $C$. Let $\tilde{L}$ be the curve in $X$ such that $\pi(\tilde{L})=R \cap Z$. Then $\tilde{R} \cap \tilde{L}$ is a point, and $\tilde{L} \subset \tilde{S}_{1}$, because otherwise we must have

$$
1=\left(\pi^{*}(3 H)-E\right) \cdot \tilde{L} \geqslant \operatorname{mult}_{\tilde{L} \cap \tilde{R}}\left(\tilde{S}_{1}\right)+\operatorname{mult}_{\tilde{L} \cap \tilde{Z}}\left(\tilde{S}_{1}\right) \geqslant 2,
$$

which is absurd. Similarly, we see that $\tilde{L} \subset \tilde{S}_{2}$, which is impossible, because we already proved that $\tilde{F}=0$. Thus, we see that $R \cap Z=\varnothing$, i.e. the lines $R$ and $Z$ are disjoint].

Let $\gamma: X \rightarrow B$ be a contraction of the extremal ray in $\overline{\mathrm{NE}}(X)$ that is generated by $\tilde{R}$. Then $\gamma$ must be a conic bundle and $B$ must be a smooth surface (see [39, Proposition 4.16]). Since $\pi(\tilde{R})$ contains the point $P$, which is a sufficiently general point in $\mathbb{P}^{3}$, we see that $\gamma$ must be a $\mathbb{P}^{1}$ bundle, which is impossible by [55]. The obtained contradiction completes the proof.

Corollary A.2 (cf. [24, Theorem 3.2]). The curve $C$ is a scheme-theoretic intersection of cubic hypersurfaces in $\mathbb{P}^{3}$.

Corollary A.3. Let $S_{1}, S_{2}$ and $S_{3}$ be three general cubic surfaces in $\mathbb{P}^{3}$ that pass through $C$. Then $S_{1} \cdot S_{2}=C+\Delta$ for an irreducible reduced conic $\Delta \subset \mathbb{P}^{3}$ such that the zero-cycle $S_{3} \cdot \Delta$ is reduced and consists of 7 distinct point in $\Delta$.

\section{REFERENCES}

[1] A. Alzati, M. Bertolini, G.-M. Besana, Projective normality of varieties of small degree, Communications in Algebra 25 (1997), 3761-3771

[2] F. Ambro, Ladders on Fano varieties J. Math. Sci., New York 94, No.1, 1126-1135 (1999).

[3] E. Arbarello, M. Cornalba, P. Griffiths, J. Harris, Geometry of algebraic curves. Volume I Grundlehren der mathematischen Wissenschaften 267 (1985) Springer-Verlag, New York-Heidelberg

[4] M. Artin, On isolated rational singularities of surfaces American Journal of Mathematics 88 (1966), 129-136

[5] G. Bordiga, La superficie del 6 ordine con 10 rette nello spazio $\mathbb{R}^{4}$ e le sue proiezioni nello spazio ordinrio Rom. Ace. L. Mem. 3 (1887), 182-203

[6] W. Bosma, J. Cannon, C. Playoust, The Magma algebra system. I. The user language Journal of Symbolic Computation, 24 (1997), 235-265

[7] E. Brieskorn, Rationale Singularitäten komplexer Flächen Invent. Math. 4, 336-358 (1968).

[8] I. Cheltsov, Log canonical thresholds of del Pezzo surfaces Geometric and Functional Analysis, 18 (2008), 1118-1144

[9] I. Cheltsov, C. Shramov, Log canonical thresholds of smooth Fano threefolds Russian Mathematical Surveys 63 (2008), 73-180

[10] I. Cheltsov, J. Park, C. Shramov, Exceptional del Pezzo hypersurfaces Journal of Geometric Analysis 20 (2010), 787-816

\footnotetext{
${ }^{7}$ As was pointed out to us by E. Ballico, the curve $\Delta$ is connected, because $C$ is arithmetically Cohen-Macaulay and thus $\Delta$ is arithmetically Cohen-Macaulay as well (see [44, Proposition 1.2]). Then $\Delta$ is a reducible conic.
} 
[11] I. Cheltsov, C. Shramov, On exceptional quotient singularities Geometry and Topology 15 (2011), 1843-1882

[12] I. Cheltsov, C. Shramov, Six-dimensional exceptional quotient singularities Mathematical Research Letters, to appear

[13] I. Cheltsov, C. Shramov, Five embeddings of one simple group Transactions of the AMS, to appear

[14] J. Conway, R. Curtis, S. Norton, R. Parker, R. Wilson, Atlas of finite groups Clarendon Press, Oxford, 1985

[15] A. Corti, Adjunction of log divisors J. Kollár (ed.). Flips and abundance for algebraic threefolds. A summer seminar at the University of Utah, Salt Lake City, 1991. Paris: Société Mathématique de France, Astérisque. 211, 171-182 (1992).

[16] J.-P. Demailly, J. Kollár, Semi-continuity of complex singularity exponents and Kähler-Einstein metrics on Fano orbifolds Annales Scientifiques de l'École Normale Supérieure 34 (2001), 525-556

[17] M. Demazure, Surfaces de Del Pezzo. I. II. III. IV. V. Semin. sur les singularites des surfaces, Cent. Math. Ec. Polytech., Palaiseau 1976-77, Lect. Notes Math. 777 (1980), 21-69.

[18] I. Dolgachev, V. Iskovskikh, Finite subgroups of the plane Cremona group Birkhauser Boston, Progress in Mathematics 269 (2009), 443-548

[19] D. Eisenbud, Commutative Algebra with a view towards Algebraic Geometry Graduate Text in Mathematics 150, Springer-Verlag (1994)

[20] D. Eisenbud, J. Harris, On varieties of minimal degree Proceedings of Symposia in Pure Mathematics 46 (1987), 3-13

[21] W. Feit, The current situation in the theory of finite simple groups Actes du Congrès International des Mathématiciens, Gauthier-Villars, Paris (1971), 55-93

[22] T. Fujita, Remarks on quasi-polarized varieties, Nagoya Mathematical Journal 115 (1989), 105-123

[23] J. Harris, Algebraic geometry: a first course Graduate Texts in Mathematics 133 (1992), Springer

[24] M. Homma, On projective normality and defining equations of a projective curve of genus three embedded by a complete linear system, Tsukuba Journal of Mathematics 4 (1980), 269-279

[25] K. Hulek, C. Okonek, A. Van de Ven, Multiplicity-2 structures on Castelnuovo surfaces Annali della Scuola Normale Superiore di Pisa 13 (1986), 427-448

[26] A. Iliev, Log-terminal singularities of algebraic surfaces Mosc. Univ. Math. Bull. 41, No.3, 46-53 (1986); translation from Vestn. Mosk. Univ., Ser. I 1986, No.3, 38-44 (1986).

[27] P. Jahnke, T. Peternell, I. Radloff, Threefolds with big and nef anticanonical bundles I Mathematische Annalen 333 (2005), 569-631

[28] P. Jahnke, T. Peternell, I. Radloff, Threefolds with big and nef anticanonical bundles II Central European Journal of Mathematics 9 (2011), 449-488

[29] Y. Kawamata, The cone of curves of algebraic varieties Ann. Math. (2) 119 (1984), 603-633

[30] Y. Kawamata, On Fujita's freeness conjecture for 3-folds and 4-folds Mathematische Annalen 308 (1997), 491-505

[31] Y. Kawamata, Subadjunction of log canonical divisors II American Journal of Mathematics 120 (1998), 893-899

[32] Y. Kawamata, K. Matsuda, K. Matsuki, Introduction to the minimal model problem Advanced Studies in Pure Mathematics 10 (1987), 283-360

[33] J. Kollár, Singularities of pairs Proceedings of Symposia in Pure Mathematics 62 (1997), 221-287

[34] S. Kudryavtsev, On purely log terminal blow ups Mathematical Notes 69 (2002), 814-819 
[35] J. Lindsey, Finite linear groups of degree six Canadian Journal of Mathematics 23 (1971), 771-790

[36] D. Markushevich, Yu. Prokhorov, Exceptional quotient singularities American Journal of Mathematics 121 (1999), 1179-1189

[37] E. Mezzetti, Differential-geometric methods for the lifting problem and linear systems on plane curves Journal of Algebraic Geometry 3 (1994), 375-398

[38] E. Mezzetti, D. Portelli, On smooth rational threefolds of $\mathbb{P}^{5}$ with rational non-special hyperplane section Mathematische Nachrichten 207 (1999), 157-182

[39] S. Mori, S. Mukai, Classifications of Fano 3-folds with $B_{2} \geqslant 2, I$ Algebraic and topological theories, Symposium dedicated to the memory of Takehiko Miyata (1985), 496-545

[40] A. Nadel, Multiplier ideal sheaves and Kähler-Einstein metrics of positive scalar curvature Annals of Mathematics 132 (1990), 549-596

[41] C. Okonek, 3-Mannigfaltigkeiten im $\mathbb{P}^{5}$ und ihre zugehörigen stabilen Garben Manuscripta Mathematica 38 (1982), 175-199

[42] C. Okonek, Über 2-codimensionale Untermannigfaltigkeiten vom Grad 7 in $\mathbb{P}^{4}$ und $\mathbb{P}^{5}$ Mathematische Zeitschrift 187 (1984), 209-219

[43] G. Ottaviani, On 3-folds in $\mathbb{P}^{5}$ which are scrolls Annali della Scuola Normale Superiore di Pisa 19 (1992), 451-471

[44] C. Peskine, L. Szpiro, Liaison des varietes algebriques. I Inventiones Mathematicae 26 (1974), 271-302

[45] Yu.Prokhorov, Blow-ups of canonical singularities Algebra (Moscow, 1998), de Gruyter, Berlin (2000), 301-317

[46] Yu. Prokhorov, Sparseness of exceptional quotient singularities Mathematical Notes 68 (2000), 664-667

[47] J. Rathmann, Double structures on Bordiga surfaces Communications in Algebra 17 (1989), 2363-2391

[48] M. Reid, Young person's guide to canonical singularities Algebraic geometry, Proc. Summer Res. Inst., Brunswick/Maine 1985, part 1, Proc. Symp. Pure Math. 46, 345-414 (1987).

[49] I. Reider, Vector bundles of rank 2 and linear system on algebraic surfaces, Annals of Mathematics 127 (1988), 309-316

[50] T. Room, The geometry of determinantal loci Cambridge University Press (1938)

[51] D. Sakovics, Weakly-exceptional quotient singularities arXiv:math.AG/1006.5909

[52] J.-P. Serre, Linear representations of finite groups Graduate Texts in Mathematics. 42. New York-Heidelberg-Berlin: Springer-Verlag. X, 170 p. (1977).

[53] V.Shokurov, Three-fold log flips Russian Academy of Sciences, Izvestiya Mathematics 40 (1993), 95-202

[54] P. Swinnerton-Dyer, An enumeration of all varieties of degree 4 American Journal of Mathematics 95 (1973), 403-418

[55] M. Szurek, J. Wiśniewski, Fano bundles of rank 2 on surfaces Compositio Mathematica 76 (1990), 295-305

[56] D. Testa, A. Varilly-Alvarado, M. Velasco, Big rational surfaces arXiv:math/0901.1094 (2009)

[57] G. Tian, On Kähler-Einstein metrics on certain Kähler manifolds with $\mathrm{c}_{1}(M)>0$ Inventiones Mathematicae 89 (1987), 225-246

[58] Q. Zhang, Rational connectedness of log $\mathbb{Q}$-Fano varietiess Journal fur die Reine und Angewandte Mathematik 590 (2006), 131-142

University of Edinburgh, Edinburgh EH9 3JZ, UK, I.Cheltsov@ed.ac.uk

Steklov Institute of Mathematics, Moscow 119991, Russia, shramov@mccme.ru

Laboratory of Algebraic Geometry, GU-HSE, 7 Vavilova Street, Moscow, 117312, Russia 